\title{
Flavor conservation in two-Higgs-doublet models
}

\author{
Francisco J. Botella* and Fernando Cornet-Gomez ${ }^{\dagger}$ \\ Departament de Física Tè̀rica and IFIC, Universitat de València-CSIC, E-46100 Burjassot, Spain \\ Miguel Nebot \\ Centro de Física Teórica de Partículas (CFTP), Instituto Superior Técnico (IST), \\ U. de Lisboa (UL), Avenida Rovisco Pais, P-1049-001 Lisboa, Portugal
}

(Received 31 May 2018; published 30 August 2018)

\begin{abstract}
In extensions of the Standard Model with two Higgs doublets, flavor-changing Yukawa couplings of the neutral scalars may be present at tree level. In this work, we consider the most general scenario in which those flavor-changing couplings are absent. We revise the conditions that the Yukawa coupling matrices must obey for such general flavour conservation ( $\mathrm{gFC}$ ) and study the one-loop renormalization group evolution of such conditions in both the quark and lepton sectors. We show that gFC in the leptonic sector is one-loop stable under the renormalization group evolution, and in the quark sector, we present some new Cabibbo-like solution also one-loop stable under renormalization group evolution. At a phenomenological level, we obtain the regions for the different $\mathrm{gFC}$ parameters that are allowed by the existing experimental constraints related to the $125 \mathrm{GeV}$ Higgs.
\end{abstract}

DOI: 10.1103/PhysRevD.98.035046

\section{INTRODUCTION}

Two-Higgs-doublet models (2HDMs) [1-3] are a simple and popular class of extensions of the Standard Model $(\mathrm{SM})$. Besides the original motivation, in particular, the possibility of having spontaneous $C P$ violation [1], extending the SM scalar sector with a second doublet allows a number of interesting phenomenological consequences. To name a few generic ones, there are the appearance of new fundamental scalar particles, nonstandard properties of the "quite Higgs-like" scalar discovered at the LHC with a mass of $125 \mathrm{GeV}$ [4,5], and, related to them, a number of potential deviations in low-energy processes with respect to SM expectations. They have been the focus of intense scrutiny before and after the 2012 discovery [6-28]. Additional aspects, including dark matter candidates $[29,30]$ or sources of $C P$ violation in addition to the Cabibbo-Kobayashi-Maskawa matrix [31-33], of interest for baryogenesis [34-36], provide further interest in 2HDMs.

In the SM, concentrating on quarks, a single Yukawa coupling matrix in each sector-up and down-is

\footnotetext{
*Francisco.J.Botella@uv.es

†Fernando.Cornet@ific.uv.es

*miguel.r.nebot.gomez@tecnico.ulisboa.pt
}

Published by the American Physical Society under the terms of the Creative Commons Attribution 4.0 International license. Further distribution of this work must maintain attribution to the author(s) and the published article's title, journal citation, and DOI. Funded by SCOAP ${ }^{3}$. responsible for both (i) the generation of mass upon spontaneous breaking of $S U(2)_{\mathrm{L}} \otimes U(1)_{\mathrm{Y}}$ into $U(1)_{\mathrm{EM}}$ and (ii) the couplings of the quarks to the only fundamental scalar leftover, the Higgs boson. As a consequence, there are no tree-level flavor-changing neutral couplings (FCNCs) of the Higgs to quarks. In the general 2HDM, there are two independent Yukawa matrices in each sector, and the situation is dramatically changed: FCNC couplings of quarks arise at tree level. Their effect on the couplings of the different physical neutral scalars depends on the details of the scalar potential [37]: if the $125 \mathrm{GeV}$ scalar is a mixture of the true-but-unphysical Higgs and the additional neutral scalars, FCNCs "leak" into its couplings through that mixing. As with many new physics avenues, the presence of FCNCs is a double-edged feature; since the competing SM gauge-mediated contributions to FCNC processes are loop induced, those transitions pose severe constraints, while on the same grounds providing immediate opportunities to discover deviations from the SM picture.

The study of different ways to avoid problematic toolarge FCNC couplings and the conditions for their appearance or absence has drawn sustained attention over the years. As analyzed in Refs. [38,39], the absence of FCNCs is guaranteed by forcing each right-handed fermion type to couple to one and only one scalar doublet; this absence of FCNCs, enforced by a $\mathbb{Z}_{2}$ symmetry, is a popular option, and several implementations of this natural flavor conservation (NFC) idea, namely, 2HDM of types I and II and of types $\mathrm{X}$ and $\mathrm{Y}$ (when the lepton sector is also considered) 
have been thoroughly explored. Additional $U(1)$ gauge symmetries have also been considered, e.g., in Refs. [40,41]. The general conditions for the absence of FCNCs, that is, that the mass matrix and the remaining Yukawa matrix can be diagonalized simultaneously, were identified early [42-45]. References [42,46-53] addressed the interplay of how a symmetry requirement could enforce that general NFC and shed some light onto the structure of the resulting Cabibbo-Kobayashi-Maskawa (CKM) matrix.

On a different line of thought, suppression (rather than the absence) of FCNCs in other "natural" manners has also attracted significant interest, including suppression given by masses like in the Cheng-Sher ansatz [54], suppression obtained from broken/approximate flavor symmetries [55-58], and symmetry controlled FCNC scenarios [59-63]. Among the latter, Branco-Grimus-Lavoura models are worth mentioning in particular, since this suppression is simply given by products of CKM matrix elements $[64,65]$ (see also related extensions [66-68]). In a more recent popular scenario, the aligned 2HDM [69], the absence of FCNCs is a priori achieved (and parametrized) with simple requirements on the Yukawa couplings (for an early mention of this kind of possibility, although in the context of real Yukawa couplings and spontaneous $C P$ violation, see also Ref. [70]). The possibility of having effective aligned scenarios has been studied in Refs. [71,72]. Radiative effects and the interplay of tree-level FCNCs with the renormalization group evolution (RGE) have also been addressed in the literature $[9,49,73-79]$.

The aim of this work is to explore different facets of scenarios with general flavor conservation $(\mathrm{gFC})$, i.e., generalized flavor alignment, in the $2 \mathrm{HDM}$, in other words, analyzing relevant aspects of the most general 2HDM scenarios in which tree-level FCNCs are, a priori, absent. An analysis of FCNCs induced in this context by the RGE has been recently presented in Ref. [80]. On a purely phenomenological basis, a scenario of this type restricted to the lepton sector was also considered in Refs. $[81,82]$.

The paper is organized as follows. In Sec. II, we revisit some generalities of the $2 \mathrm{HDM}$, fix the notation for the discussion to follow, and recall the most relevant aspects of the conditions leading to gFC. They are then analyzed, attending to the renormalization group evolution that they obey in Sec. III, leading to the full set of conditions required to have RGE-stable gFC. The well-known type-I and type-II cases are briefly revisited in Sec. III C; Sec. III $\mathrm{E}$ is devoted to a particular solution that arises when the CKM matrix is reduced to a single Cabibbo-like mixing. The gFC stability of the lepton sector is discussed in Sec. III D. In Sec. IV, we discuss the most relevant experimental constraints on $\mathrm{gFC}$ arising from flavor-conserving Higgs-related observables, leading to the analysis and results of Sec. IV D. In Sec. IVE, we provide, for comparison, results for the usual 2HDMs of types I, II, X, and $\mathrm{Y}$ and for the aligned 2HDM.
Appendix provides details omitted in the discussion of Sec. III.

\section{YUKAWA COUPLINGS AND GENERAL FLAVOR CONSERVATION}

The Higgs doublets $(j=1,2)$ of the $2 \mathrm{HDM}$ are

$$
\Phi_{j}=e^{i \theta_{j}}\left(\begin{array}{c}
\varphi_{j}^{+} \\
\left(v_{j}+\rho_{j}+i \eta_{j}\right) / \sqrt{2}
\end{array}\right),
$$

where $v_{j}$ and $\theta_{j}$ are real numbers, $\rho_{j}$ and $\eta_{j}$ are neutral (Hermitian) fields, and $\varphi_{j}^{ \pm}$are charged fields. Equation (1) anticipates the assumption that the scalar potential $V\left(\Phi_{1}, \Phi_{2}\right)[2,3]$ is such that $V\left(\left\langle\Phi_{1}\right\rangle,\left\langle\Phi_{2}\right\rangle\right)$ has an appropriate minimum at

$$
\left\langle\Phi_{j}\right\rangle=\left(\begin{array}{c}
0 \\
e^{i \theta_{j}} v_{j} / \sqrt{2}
\end{array}\right) .
$$

In the "Higgs basis" [7,37,83], only one linear combination of $\Phi_{1}$ and $\Phi_{2}, H_{1}$, has a nonvanishing vacuum expectation value,

$$
\begin{aligned}
\left(\begin{array}{l}
H_{1} \\
H_{2}
\end{array}\right) & =\mathcal{R}_{\beta}\left(\begin{array}{c}
e^{-i \theta_{1}} \Phi_{1} \\
e^{-i \theta_{2}} \Phi_{2}
\end{array}\right), \quad\left\langle H_{1}\right\rangle=\frac{v}{\sqrt{2}}\left(\begin{array}{l}
0 \\
1
\end{array}\right), \\
\left\langle H_{2}\right\rangle & =\left(\begin{array}{l}
0 \\
0
\end{array}\right)
\end{aligned}
$$

with $v=\sqrt{v_{1}^{2}+v_{2}^{2}}, \quad c_{\beta}=\cos \beta \equiv v_{1} / v, s_{\beta}=\sin \beta=v_{2} / v$, $t_{\beta}=s_{\beta} / c_{\beta}$, and

$$
\mathcal{R}_{\beta} \equiv\left(\begin{array}{cc}
c_{\beta} & s_{\beta} \\
-s_{\beta} & c_{\beta}
\end{array}\right), \quad\left[\mathcal{R}_{\beta}\right]^{-1}=\left[\mathcal{R}_{\beta}\right]^{T} .
$$

The expansion of $H_{1}$ and $H_{2}$ around that minimum of the potential reads

$$
\begin{aligned}
& H_{1}=\left(\begin{array}{c}
G^{+} \\
\left(v+\mathrm{H}^{0}+i G^{0}\right) / \sqrt{2}
\end{array}\right), \\
& H_{2}=\left(\begin{array}{c}
\mathrm{H}^{+} \\
\left(\mathrm{R}^{0}+i \mathrm{I}^{0}\right) / \sqrt{2}
\end{array}\right),
\end{aligned}
$$

where

$$
\begin{aligned}
\left(\begin{array}{c}
G^{+} \\
\mathrm{H}^{+}
\end{array}\right) & =\mathcal{R}_{\beta}\left(\begin{array}{c}
\varphi_{1}^{+} \\
\varphi_{2}^{+}
\end{array}\right), \quad\left(\begin{array}{c}
G^{0} \\
\mathrm{I}^{0}
\end{array}\right)=\mathcal{R}_{\beta}\left(\begin{array}{l}
\eta_{1} \\
\eta_{2}
\end{array}\right), \\
\left(\begin{array}{l}
\mathrm{H}^{0} \\
\mathrm{R}^{0}
\end{array}\right) & =\mathcal{R}_{\beta}\left(\begin{array}{c}
\rho_{1} \\
\rho_{2}
\end{array}\right) .
\end{aligned}
$$

The would-be Goldstone bosons $G^{0}$ and $G^{ \pm}$provide the longitudinal degrees of freedom of the $Z$ and $W^{ \pm}$gauge 
bosons; furthermore, while $\mathrm{H}^{ \pm}$is already a physical charged scalar field, the physical neutral scalars $\{\mathrm{h}, \mathrm{H}, \mathrm{A}\}$ are real linear combinations of $\left\{\mathrm{H}^{0}, \mathrm{R}^{0}, \mathrm{I}^{0}\right\}$,

$\mathcal{R}_{[3]}(\vec{\alpha})\left(\begin{array}{c}\mathrm{h} \\ \mathrm{H} \\ \mathrm{A}\end{array}\right)=\left(\begin{array}{c}\mathrm{H}^{0} \\ \mathrm{R}^{0} \\ \mathrm{I}^{0}\end{array}\right), \quad\left[\mathcal{R}_{[3]}(\vec{\alpha})\right]^{-1}=\left[\mathcal{R}_{[3]}(\vec{\alpha})\right]^{T}$,

with $\left[\mathcal{R}_{[3]}(\vec{\alpha})\right]$ a real orthogonal rotation described by three real mixing angles, $\vec{\alpha}=\left\{\alpha_{12}, \alpha_{13}, \alpha_{23}\right\}\left(c_{x} \equiv \cos x\right.$, $\left.s_{x} \equiv \sin x\right)$,

$$
\begin{aligned}
{\left[\mathcal{R}_{[3]}(\vec{\alpha})\right]=} & \left(\begin{array}{ccc}
c_{\alpha_{12}} & -s_{\alpha_{12}} & 0 \\
s_{\alpha_{12}} & c_{\alpha_{12}} & 0 \\
0 & 0 & 1
\end{array}\right)\left(\begin{array}{ccc}
c_{\alpha_{13}} & 0 & -s_{\alpha_{13}} \\
0 & 1 & 0 \\
s_{\alpha_{13}} & 0 & c_{\alpha_{13}}
\end{array}\right) \\
& \times\left(\begin{array}{ccc}
1 & 0 & 0 \\
0 & c_{\alpha_{23}} & -s_{\alpha_{23}} \\
0 & s_{\alpha_{23}} & c_{\alpha_{23}}
\end{array}\right) .
\end{aligned}
$$

When there is no $C P$ violation in the scalar potential, i.e., no mixing connecting the $C P$-even $\mathrm{H}^{0}$ and $\mathrm{R}^{0}$ and the $C P$-odd $\mathrm{I}^{0}$, it is customary to introduce the mixing angle $\alpha$,

$$
\left(\begin{array}{c}
\mathrm{h} \\
\mathrm{H}
\end{array}\right)=\left(\begin{array}{cc}
s_{\alpha} & c_{\alpha} \\
-c_{\alpha} & s_{\alpha}
\end{array}\right)\left(\begin{array}{c}
\rho_{1} \\
\rho_{2}
\end{array}\right)=\left(\begin{array}{cc}
s_{\beta \alpha} & c_{\beta \alpha} \\
-c_{\beta \alpha} & s_{\beta \alpha}
\end{array}\right)\left(\begin{array}{c}
\mathrm{H}^{0} \\
\mathrm{R}^{0}
\end{array}\right),
$$

where $s_{\beta \alpha}=\sin (\alpha+\beta)$ and $c_{\beta \alpha}=\cos (\alpha+\beta)$ [that is, $\alpha_{13}=\alpha_{23}=0$ and $\alpha_{12}=\pi / 2-(\alpha+\beta)$ in Eq. (8)]. Since a \pm sign can be included in the definition of the scalar fields without changing their kinetic terms, different conventions for Eqs. (8) and (9), which may be relevant when comparing expressions, are used in the literature.

\section{A. Quark Yukawa couplings in 2HDM}

The Yukawa couplings of the quarks- $S U(2)_{L}$ doublets $Q_{L}^{0}$ and singlets $d_{R}^{0}, u_{R}^{0}$-with the scalar doublets read

$$
\begin{aligned}
\mathcal{L}_{\mathrm{Y}}^{[q]}= & -\bar{Q}_{L}^{0}\left[\Gamma_{1} \Phi_{1}+\Gamma_{2} \Phi_{2}\right] d_{R}^{0} \\
& -\bar{Q}_{L}^{0}\left[\Delta_{1} \tilde{\Phi}_{1}+\Delta_{2} \tilde{\Phi}_{2}\right] u_{R}^{0}+\text { H.c. }
\end{aligned}
$$

with $\tilde{\Phi}_{j}=i \sigma_{2} \Phi_{j}^{*}$. Following Eqs. (3)-(5),

$$
\begin{aligned}
\mathcal{L}_{\mathrm{Y}}^{[q]}= & -\frac{\sqrt{2}}{v} \bar{Q}_{L}^{0}\left[\mathcal{M}_{\mathrm{d}}^{0} H_{1}+\mathcal{N}_{\mathrm{d}}^{0} H_{2}\right] d_{R}^{0} \\
& -\frac{\sqrt{2}}{v} \bar{Q}_{L}^{0}\left[\mathcal{M}_{\mathrm{u}}^{0} \tilde{H}_{1}+\mathcal{N}_{\mathrm{u}}^{0} \tilde{H}_{2}\right] u_{R}^{0}+\text { H.c. } \\
= & \mathcal{L}_{\mathrm{m}}^{[q]}+\mathcal{L}_{\mathrm{G}}^{[q]}+\mathcal{L}_{\mathrm{Ch}}^{[q]}+\mathcal{L}_{\mathrm{N}}^{[q]}
\end{aligned}
$$

with mass terms $\mathcal{L}_{\mathrm{m}}^{[q]}$, would-be Goldstone boson couplings $\mathcal{L}_{\mathrm{G}}^{[q]}$, and Yukawa couplings to charged and neutral scalars, $\mathcal{L}_{\mathrm{Ch}}^{[q]}$ and $\mathcal{L}_{\mathrm{N}}^{[q]}$ :

$$
\begin{aligned}
\mathcal{L}_{\mathrm{m}}^{[q]} \supset & -\bar{d}_{L}^{0} \mathcal{M}_{\mathrm{d}}^{0} d_{R}^{0}-\bar{u}_{L}^{0} \mathcal{M}_{\mathrm{u}}^{0} u_{R}^{0}, \\
\mathcal{L}_{\mathrm{G}}^{[q]} \supset & -\frac{\sqrt{2}}{v}\left[G^{+} \bar{u}_{L}^{0} \mathcal{M}_{\mathrm{d}}^{0} d_{R}^{0}+i G^{0} \bar{d}_{L}^{0} \mathcal{M}_{\mathrm{d}}^{0} d_{R}^{0}\right. \\
& \left.-G^{-} \bar{d}_{L}^{0} \mathcal{M}_{\mathrm{u}}^{0} u_{R}^{0}-i G^{0} \bar{u}_{L}^{0} \mathcal{M}_{\mathrm{u}}^{0} u_{R}^{0}\right], \\
\mathcal{L}_{\mathrm{Ch}}^{[q]} \supset & -\frac{\sqrt{2}}{v}\left[\mathrm{H}^{+} \bar{u}_{L}^{0} \mathcal{N}_{\mathrm{d}}^{0} d_{R}^{0}-\mathrm{H}^{-} \bar{d}_{L}^{0} \mathcal{N}_{\mathrm{u}}^{0} u_{R}^{0}\right], \\
\mathcal{L}_{\mathrm{N}}^{[q]} \supset & -\frac{1}{v}\left[\mathrm{H}^{0} \bar{d}_{L}^{0} \mathcal{M}_{\mathrm{d}}^{0} d_{R}^{0}+\left(\mathrm{R}^{0}+i \mathrm{I}^{0}\right) \bar{d}_{L}^{0} \mathcal{N}_{\mathrm{d}}^{0} d_{R}^{0}\right. \\
& \left.+\mathrm{H}^{0} \bar{u}_{L}^{0} \mathcal{M}_{\mathrm{u}}^{0} u_{R}^{0}+\left(\mathrm{R}^{0}-i \mathrm{I}^{0}\right) \bar{u}_{L}^{0} \mathcal{N}_{\mathrm{u}}^{0} u_{R}^{0}\right] .
\end{aligned}
$$

The mass matrices are

$$
\begin{aligned}
& \mathcal{M}_{\mathrm{d}}^{0}=\frac{v}{\sqrt{2}}\left[e^{i \theta_{1}} c_{\beta} \Gamma_{1}+e^{i \theta_{2}} s_{\beta} \Gamma_{2}\right], \\
& \mathcal{M}_{\mathrm{u}}^{0}=\frac{v}{\sqrt{2}}\left[e^{-i \theta_{1}} c_{\beta} \Delta_{1}+e^{-i \theta_{2}} s_{\beta} \Delta_{2}\right],
\end{aligned}
$$

and the second linear combinations of Yukawa matrices that encode the potential FCNCs are

$$
\begin{aligned}
& \mathcal{N}_{\mathrm{d}}^{0}=\frac{v}{\sqrt{2}}\left[-e^{i \theta_{1}} s_{\beta} \Gamma_{1}+e^{i \theta_{2}} c_{\beta} \Gamma_{2}\right], \\
& \mathcal{N}_{\mathrm{u}}^{0}=\frac{v}{\sqrt{2}}\left[-e^{-i \theta_{1}} s_{\beta} \Delta_{1}+e^{-i \theta_{2}} c_{\beta} \Delta_{2}\right] .
\end{aligned}
$$

For the usual bidiagonalization of the mass matrices $\mathcal{M}_{\mathrm{d}}^{0}$ and $\mathcal{M}_{\mathrm{u}}^{0}$, the quark mass eigenstates (without a 0 superscript) read

$d_{L}=U_{\mathrm{d}_{\mathrm{L}}}^{\dagger} d_{L}^{0}, \quad d_{R}=U_{\mathrm{d}_{\mathrm{R}}}^{\dagger} d_{R}^{0}, \quad u_{L}=U_{\mathrm{u}_{\mathrm{L}}}^{\dagger} u_{L}^{0}, \quad u_{R}=U_{\mathrm{u}_{\mathrm{R}}}^{\dagger} u_{R}^{0}$,

with

$$
\begin{aligned}
& \mathcal{M}_{\mathrm{d}}=U_{\mathrm{d}_{\mathrm{L}}}^{\dagger} \mathcal{M}_{\mathrm{d}}^{0} U_{\mathrm{d}_{\mathrm{R}}}=\operatorname{diag}\left(m_{d}, m_{s}, m_{b}\right), \\
& \mathcal{M}_{\mathrm{u}}=U_{\mathrm{u}_{\mathrm{L}}}^{\dagger} \mathcal{M}_{\mathrm{u}}^{0} U_{\mathrm{u}_{\mathrm{R}}}=\operatorname{diag}\left(m_{u}, m_{c}, m_{t}\right), \\
& \mathcal{N}_{\mathrm{d}}=U_{\mathrm{d}_{\mathrm{L}}}^{\dagger} \mathcal{N}_{\mathrm{d}}^{0} U_{\mathrm{d}_{\mathrm{R}}}, \quad \mathcal{N}_{\mathrm{u}}=U_{\mathrm{u}_{\mathrm{L}}}^{\dagger} \mathcal{N}_{\mathrm{u}}^{0} U_{\mathrm{u}_{\mathrm{R}}} .
\end{aligned}
$$

The CKM matrix is $V=U_{\mathrm{u}_{\mathrm{L}}}^{\dagger} U_{\mathrm{d}_{\mathrm{L}}}$. When both $\mathcal{N}_{\mathrm{d}}$ and $\mathcal{N}_{\mathrm{u}}$ are diagonal, tree-level FCNCs are absent.

Expressing Eq. (11) in terms of quark and scalar mass eigenstates (for a shorthand, we use $\left[\mathcal{R}_{[3]}(\vec{\alpha})\right]_{i j}=\mathcal{R}_{i j}$ ), 


$$
\begin{aligned}
\mathcal{L}_{\mathrm{m}}^{[q]}= & -\bar{d}_{L} \mathcal{M}_{\mathrm{d}} d_{R}-\bar{u}_{L} \mathcal{M}_{\mathrm{u}} u_{R}+\text { H.c. }, \\
\mathcal{L}_{\mathrm{G}}^{[q]}= & -\frac{\sqrt{2}}{v}\left[G^{+} \bar{u}_{L} V \mathcal{M}_{\mathrm{d}} d_{R}+i G^{0} \bar{d}_{L} \mathcal{M}_{\mathrm{d}} d_{R}+G^{-} \bar{d}_{L} V^{\dagger} \mathcal{M}_{\mathrm{u}} u_{R}-i G^{0} \bar{u}_{L} \mathcal{M}_{\mathrm{u}} u_{R}\right]+\mathrm{H} . \mathrm{c} ., \\
\mathcal{L}_{\mathrm{Ch}}^{[q]}= & -\frac{\sqrt{2}}{v}\left\{\mathrm{H}^{+}\left[\bar{u}_{L} V \mathcal{N}_{\mathrm{d}} d_{R}-\bar{u}_{R} \mathcal{N}^{\dagger}{ }_{\mathrm{u}} V d_{L}\right]+\mathrm{H}^{-}\left[\bar{d}_{R} \mathcal{N}_{\mathrm{d}}^{\dagger} V^{\dagger} u_{L}-\bar{d}_{L} V^{\dagger} \mathcal{N}_{\mathrm{u}} u_{R}\right]\right\} \\
\mathcal{L}_{\mathrm{N}}^{[q]}= & -\frac{\mathrm{h}}{v}\left\{\bar{d}\left[\mathcal{R}_{11} \mathcal{M}_{\mathrm{d}}+\mathcal{R}_{21} \mathcal{H}_{\mathrm{d}}+i \mathcal{R}_{31} \mathcal{A}_{\mathrm{d}}\right] d+\bar{d}\left[\mathcal{R}_{21} \mathcal{A}_{\mathrm{d}}+i \mathcal{R}_{31} \mathcal{H}_{\mathrm{d}}\right] \gamma_{5} d\right\} \\
& -\frac{\mathrm{h}}{v}\left\{\bar{u}\left[\mathcal{R}_{11} \mathcal{M}_{\mathrm{u}}+\mathcal{R}_{21} \mathcal{H}_{\mathrm{u}}-i \mathcal{R}_{31} \mathcal{A}_{\mathrm{u}}\right] u+\bar{u}\left[\mathcal{R}_{21} \mathcal{A}_{\mathrm{u}}-i \mathcal{R}_{31} \mathcal{H}_{\mathrm{u}}\right] \gamma_{5} u\right\} \\
& -\frac{\mathrm{H}}{v}\left\{\bar{d}\left[\mathcal{R}_{12} \mathcal{M}_{\mathrm{d}}+\mathcal{R}_{22} \mathcal{H}_{\mathrm{d}}+i \mathcal{R}_{32} \mathcal{A}_{\mathrm{d}}\right] d+\bar{d}\left[\mathcal{R}_{22} \mathcal{A}_{\mathrm{d}}+i \mathcal{R}_{32} \mathcal{H}_{\mathrm{d}}\right] \gamma_{5} d\right\} \\
& -\frac{\mathrm{H}}{v}\left\{\bar{u}\left[\mathcal{R}_{12} \mathcal{M}_{\mathrm{u}}+\mathcal{R}_{22} \mathcal{H}_{\mathrm{u}}-i \mathcal{R}_{32} \mathcal{A}_{\mathrm{u}}\right] u+\bar{u}\left[\mathcal{R}_{22} \mathcal{A}_{\mathrm{u}}-i \mathcal{R}_{32} \mathcal{H}_{\mathrm{u}}\right] \gamma_{5} u\right\} \\
& -\frac{\mathrm{A}}{v}\left\{\bar{d}\left[\mathcal{R}_{13} \mathcal{M}_{\mathrm{d}}+\mathcal{R}_{23} \mathcal{H}_{\mathrm{d}}+i \mathcal{R}_{33} \mathcal{A}_{\mathrm{d}}\right] d+\bar{d}\left[\mathcal{R}_{23} \mathcal{A}_{\mathrm{d}}+i \mathcal{R}_{33} \mathcal{H}_{\mathrm{d}}\right] \gamma_{5} d\right\} \\
& -\frac{\mathrm{A}}{v}\left\{\bar{u}\left[\mathcal{R}_{13} \mathcal{M}_{\mathrm{u}}+\mathcal{R}_{23} \mathcal{H}_{\mathrm{u}}-i \mathcal{R}_{33} \mathcal{A}_{\mathrm{u}}\right] u+\bar{u}\left[\mathcal{R}_{23} \mathcal{A}_{\mathrm{u}}-i \mathcal{R}_{33} \mathcal{H}_{\mathrm{u}}\right] \gamma_{5} u\right\}
\end{aligned}
$$

where

$\mathcal{H}_{\mathrm{q}} \equiv \frac{\mathcal{N}_{\mathrm{q}}+\mathcal{N}_{\mathrm{q}}^{\dagger}}{2}, \quad \mathcal{A}_{\mathrm{q}} \equiv \frac{\mathcal{N}_{\mathrm{q}}-\mathcal{N}_{\mathrm{q}}^{\dagger}}{2}, \quad q=u, d$,

are the Hermitian and anti-Hermitian combinations of $\mathcal{N}_{\mathrm{q}}$ and $\mathcal{N}^{\dagger}$ q.

With no $C P$ violation in the scalar sector,

$$
\mathcal{R}=\left(\begin{array}{ccc}
s_{\beta \alpha} & -c_{\beta \alpha} & 0 \\
c_{\beta \alpha} & s_{\beta \alpha} & 0 \\
0 & 0 & 1
\end{array}\right)
$$

and Eq. (25) reduces to

$$
\begin{aligned}
\mathcal{L}_{\mathrm{N}}^{[q]}= & -\frac{\mathrm{h}}{v}\left\{\bar{d}\left[s_{\beta \alpha} \mathcal{M}_{\mathrm{d}}+c_{\beta \alpha} \mathcal{H}_{\mathrm{d}}\right] d+c_{\beta \alpha} \bar{d} \mathcal{A}_{\mathrm{d}} \gamma_{5} d\right\} \\
& -\frac{\mathrm{h}}{v}\left\{\bar{u}\left[s_{\beta \alpha} \mathcal{M}_{\mathrm{u}}+c_{\beta \alpha} \mathcal{H}_{\mathrm{u}}\right] u+c_{\beta \alpha} \bar{u} \mathcal{A}_{\mathrm{u}} \gamma_{5} u\right\} \\
& -\frac{\mathrm{H}}{v}\left\{\bar{d}\left[-c_{\beta \alpha} \mathcal{M}_{\mathrm{d}}+s_{\beta \alpha} \mathcal{H}_{\mathrm{d}}\right] d+s_{\beta \alpha} \bar{d} \mathcal{A}_{\mathrm{d}} \gamma_{5} d\right\} \\
& -\frac{\mathrm{H}}{v}\left\{\bar{u}\left[-c_{\beta \alpha} \mathcal{M}_{\mathrm{u}}+s_{\beta \alpha} \mathcal{H}_{\mathrm{u}}\right] u+s_{\beta \alpha} \bar{u} \mathcal{A}_{\mathrm{u}} \gamma_{5} u\right\} \\
& -i \frac{\mathrm{A}}{v}\left\{\bar{d} \mathcal{A}_{\mathrm{d}} d+\bar{d} \mathcal{H}_{\mathrm{d}} \gamma_{5} d\right\}+i \frac{\mathrm{A}}{v}\left\{\bar{u} \mathcal{A}_{\mathrm{u}} u+\bar{u} \mathcal{H}_{\mathrm{u}} \gamma_{5} u\right\} .
\end{aligned}
$$

\section{B. Lepton Yukawa couplings in $2 \mathrm{HDM}$}

The Yukawa couplings of the lepton $S U(2)_{L}$ doublets $L_{L}^{0}$ and singlets $\ell_{R}^{0}$ with the scalar doublets are

$$
\begin{aligned}
\mathcal{L}_{\mathrm{Y}}^{[\ell]} & =-\bar{L}_{L}^{0}\left(\Pi_{1} \Phi_{1}+\Pi_{2} \Phi_{2}\right) \ell_{R}^{0}+\text { H.c. } \\
& =-\frac{\sqrt{2}}{v} \bar{L}_{L}^{0}\left(\mathcal{M}_{\ell}^{0} H_{1}+\mathcal{N}_{\ell}^{0} H_{2}\right) \ell_{R}^{0}+\text { H.c. },
\end{aligned}
$$

where, similarly to the quark sector in the previous section,

$$
\begin{aligned}
& \mathcal{M}_{\ell}^{0}=\frac{v}{\sqrt{2}}\left[e^{i \theta_{1}} c_{\beta} \Pi_{1}+e^{i \theta_{2}} s_{\beta} \Pi_{2}\right], \\
& \mathcal{N}_{\ell}^{0}=\frac{v}{\sqrt{2}}\left[-e^{i \theta_{1}} s_{\beta} \Pi_{1}+e^{i \theta_{2}} c_{\beta} \Pi_{2}\right] .
\end{aligned}
$$

The mass eigenstates, without a 0 superscript, correspond to

$$
\ell_{L}=U_{\ell_{L}}^{\dagger} \ell_{L}^{0}, \quad \ell_{R}=U_{\ell_{R}}^{\dagger} \ell_{R}^{0},
$$

and

$$
\begin{aligned}
& \mathcal{M}_{\ell}=U_{\ell_{L}}^{\dagger} \mathcal{M}_{\ell}^{0} U_{\ell_{R}}=\operatorname{diag}\left(m_{e}, m_{\mu}, m_{\tau}\right), \\
& \mathcal{N}_{\ell}=U_{\ell_{L}}^{\dagger} \mathcal{N}_{\ell}^{0} U_{\ell_{R}} .
\end{aligned}
$$

Notice that we do not include right-handed neutrinos $\nu_{R}^{0}$ and thus, unlike in the quark sector, there is only one set of Yukawa coupling matrices and we work in the massless neutrino approximation. The leptonic analogs of the Yukawa couplings in Eqs. (24) and (25) are 


$$
\begin{aligned}
\mathcal{L}_{\mathrm{Ch}}^{[\ell]}= & -\frac{\sqrt{2}}{v}\left\{\mathrm{H}^{+} \bar{\nu}_{L} \mathcal{N}_{\ell} \ell_{R}+\mathrm{H}^{-} \bar{\ell}_{R} \mathcal{N}^{\dagger} \ell \nu_{L}\right\}, \\
\mathcal{L}_{\mathrm{N}}^{[\ell]}= & -\frac{\mathrm{h}}{v}\left\{\bar{\ell}\left[\mathcal{R}_{11} \mathcal{M}_{\ell}+\mathcal{R}_{21} \mathcal{H}_{\ell}+i \mathcal{R}_{31} \mathcal{A}_{\ell}\right] \ell+\bar{\ell}\left[\mathcal{R}_{21} \mathcal{A}_{\ell}+i \mathcal{R}_{31} \mathcal{H}_{\ell}\right] \gamma_{5} \ell\right\} \\
& -\frac{\mathrm{H}}{v}\left\{\bar{\ell}\left[\mathcal{R}_{12} \mathcal{M}_{\ell}+\mathcal{R}_{22} \mathcal{H}_{\ell}+i \mathcal{R}_{32} \mathcal{A}_{\ell}\right] \ell+\bar{\ell}\left[\mathcal{R}_{22} \mathcal{A}_{\ell}+i \mathcal{R}_{32} \mathcal{H}_{\ell}\right] \gamma_{5} \ell\right\} \\
& -\frac{\mathrm{A}}{v}\left\{\bar{\ell}\left[\mathcal{R}_{13} \mathcal{M}_{\ell}+\mathcal{R}_{23} \mathcal{H}_{\ell}+i \mathcal{R}_{33} \mathcal{A}_{\ell}\right] \ell+\bar{\ell}\left[\mathcal{R}_{23} \mathcal{A}_{\ell}+i \mathcal{R}_{33} \mathcal{H}_{\ell}\right] \gamma_{5} \ell\right\},
\end{aligned}
$$

with

$$
\mathcal{H}_{\ell} \equiv \frac{\mathcal{N}_{\ell}+\mathcal{N}^{\dagger} \ell}{2}, \quad \mathcal{A}_{\ell} \equiv \frac{\mathcal{N}_{\ell}-\mathcal{N}^{\dagger} \ell}{2}
$$

\section{General flavor conservation}

The necessary and sufficient conditions obeyed by the quark Yukawa coupling matrices $\Gamma_{\alpha}, \Delta_{\alpha}, \alpha=1,2$, in order to have gFC [42-45], are that each of the sets

$$
\left\{\Gamma_{\alpha} \Gamma_{\beta}^{\dagger}\right\}, \quad\left\{\Gamma_{\alpha}^{\dagger} \Gamma_{\beta}\right\}, \quad\left\{\Delta_{\alpha} \Delta_{\beta}^{\dagger}\right\}, \quad\left\{\Delta_{\alpha}^{\dagger} \Delta_{\beta}\right\}, \quad \alpha, \beta=1,2
$$

is Abelian; that is, their elements commute,

$$
\begin{aligned}
{\left[\Gamma_{\alpha} \Gamma_{\beta}^{\dagger}, \Gamma_{\gamma} \Gamma_{\delta}^{\dagger}\right] } & =0, & & {\left[\Gamma_{\alpha}^{\dagger} \Gamma_{\beta}, \Gamma_{\gamma}^{\dagger} \Gamma_{\delta}\right]=0, } \\
{\left[\Delta_{\alpha} \Delta_{\beta}^{\dagger}, \Delta_{\gamma} \Delta_{\delta}^{\dagger}\right] } & =0, & & {\left[\Delta_{\alpha}^{\dagger} \Delta_{\beta}, \Delta_{\gamma}^{\dagger} \Delta_{\delta}\right]=0, }
\end{aligned}
$$

with $\alpha, \beta, \gamma, \delta=1,2$. In that case, $\left\{\Gamma_{1}, \Gamma_{2}\right\}$ are simultaneously bidiagonalized, and $\left\{\Delta_{1}, \Delta_{2}\right\}$, too.

A crucial corollary to these necessary and sufficient conditions is the fact that the simultaneous diagonalizability is intrinsic to the Yukawa coupling matrices themselves, independently of the spontaneous symmetry breaking vacuum characterized by the vacuum expectation values $v_{1}, v_{2}$. In other words, the property is independent of $\beta$ in Eqs. (17) and (18); the simultaneous bidiagonalizability of $\left\{\mathcal{M}_{\mathrm{q}}^{0}, \mathcal{N}_{\mathrm{q}}^{0}\right\}$ is equivalent to the simultaneous bidiagonalizability of the Yukawa couplings matrices or of any other independent linear combinations of them. Of course, the actual values of the eigenvalues of both $\mathcal{M}_{\mathrm{q}}^{0}$ (the masses) and $\mathcal{N}_{\mathrm{q}}^{0}$ do depend on the particular linear combinations.

For leptons, similarly, $\left\{\Pi_{\alpha} \Pi_{\beta}^{\dagger}\right\}$ and $\left\{\Pi_{\alpha}^{\dagger} \Pi_{\beta}\right\}$ must be Abelian in order to have $\mathrm{gFC}$, and the previous corollary applies equally to them.

A very relevant consequence follows [42,46-53]: if $\mathrm{gFC}$ is due to the Lagrangian in Eq. (10) being invariant under a (symmetry) transformation of quarks and scalars, the CKM mixing matrix cannot be related to the values of the masses; e.g., predictions being made at the time (the late 1970s) ${ }^{1}$ for the Cabibbo angle, like $\tan \theta_{c}=$ $m_{d} / m_{s}[84,85]$, could not lead simultaneously to $g F C$. Moreover, the resulting mixings are unrealistic (e.g., no mixing or a permutation times a complex phase), and radiative corrections cannot be invoked to yield realistic mixings [49].

The most general parametrization of tree-level couplings of fermions to scalars obeying $\mathrm{gFC}$ is, quite trivially,

$$
\begin{array}{ll}
\mathcal{N}_{\mathrm{d}}=\left(\begin{array}{ccc}
n_{d} & 0 & 0 \\
0 & n_{s} & 0 \\
0 & 0 & n_{b}
\end{array}\right), & \mathcal{N}_{\mathrm{u}}=\left(\begin{array}{ccc}
n_{u} & 0 & 0 \\
0 & n_{c} & 0 \\
0 & 0 & n_{t}
\end{array}\right), \\
\mathcal{N}_{\ell}=\left(\begin{array}{ccc}
n_{e} & 0 & 0 \\
0 & n_{\mu} & 0 \\
0 & 0 & n_{\tau}
\end{array}\right), & n_{j} \in \mathbb{C},
\end{array}
$$

which we use in the rest of the paper: in Sec. III for the study of the renormalization group evolution and in Sec. IV for a phenomenological analysis.

Notice that, while for the flavor-changing couplings the simultaneous presence of scalar and pseudoscalar terms in fermion-scalar Yukawa interactions is not necessarily $C P$ violating, in the diagonal, flavor-conserving ones, on the contrary, it is $C P$ violating (see, e.g., Ref. [86]). With the flavor-conserving matrices $\mathcal{N}_{\mathrm{f}}$ in Eq. (38), the Hermitian and anti-Hermitian couplings in Eqs. (28) and (34) are, respectively, their real and imaginary parts. For example, for a $C P$-conserving scalar sector with nonzero mixing $c_{\beta \alpha} \neq 0$, if $\mathcal{N}_{\mathrm{f}}$ are not real, they constitute new sources of $C P$ violation in neutral couplings. For the couplings to the charged scalar, without entering into details, if $\operatorname{Im}\left(n_{u_{i}} n_{d_{j}}\right) \neq 0$, the combination of scalar and pseudoscalar terms in the coupling $\mathrm{H}^{+} \bar{u}_{i} d_{j}$ is $C P$ violating.

\footnotetext{
${ }^{1}$ In the context of $S U(2)_{L} \otimes U(1)_{Y}$ gauge theories; the literature is richer in examples for $S U(2)_{L} \otimes S U(2)_{R} \otimes U(1)_{Y}$ scenarios.
} 


\section{RENORMALIZATION GROUP EVOLUTION AND FLAVOR CONSERVATION}

\section{A. Evolution of the quark Yukawa coupling matrices}

The one-loop evolution of the Yukawa couplings under the renormalization group [75-77,87] is (with $\mathcal{D} \equiv 16 \pi^{2} \frac{d}{d \ln \mu}$ and $\mu$ the energy scale)

$$
\begin{aligned}
\mathcal{D} \Gamma_{\alpha}= & a_{d} \Gamma_{\alpha}+\sum_{\rho=1}^{n=2} T_{\alpha, \rho}^{d} \Gamma_{\rho}+\sum_{\rho=1}^{n=2}\left(-2 \Delta_{\rho} \Delta_{\alpha}^{\dagger} \Gamma_{\rho}+\Gamma_{\alpha} \Gamma_{\rho}^{\dagger} \Gamma_{\rho}+\frac{1}{2} \Delta_{\rho} \Delta_{\rho}^{\dagger} \Gamma_{\alpha}+\frac{1}{2} \Gamma_{\rho} \Gamma_{\rho}^{\dagger} \Gamma_{\alpha}\right) \\
& \text { with } \quad T_{\alpha, \rho}^{d} \equiv 3 \operatorname{tr}\left(\Gamma_{\alpha} \Gamma_{\rho}^{\dagger}+\Delta_{\alpha}^{\dagger} \Delta_{\rho}\right)+\operatorname{tr}\left(\Pi_{\alpha} \Pi_{\rho}^{\dagger}\right), \\
\mathcal{D} \Delta_{\alpha}= & a_{u} \Delta_{\alpha}+\sum_{\rho=1}^{n=2} T_{\alpha, \rho}^{u} \Delta_{\rho}+\sum_{\rho=1}^{n=2}\left(-2 \Gamma_{\rho} \Gamma_{\alpha}^{\dagger} \Delta_{\rho}+\Delta_{\alpha} \Delta_{\rho}^{\dagger} \Delta_{\rho}+\frac{1}{2} \Gamma_{\rho} \Gamma_{\rho}^{\dagger} \Delta_{\alpha}+\frac{1}{2} \Delta_{\rho} \Delta_{\rho}^{\dagger} \Delta_{\alpha}\right) \\
& \text { with } \quad T_{\alpha, \rho}^{u} \equiv 3 \operatorname{tr}\left(\Delta_{\alpha} \Delta_{\rho}^{\dagger}+\Gamma_{\alpha}^{\dagger} \Gamma_{\rho}\right)+\operatorname{tr}\left(\Pi_{\alpha}^{\dagger} \Pi_{\rho}\right)=T_{\alpha, \rho}^{d *},
\end{aligned}
$$

where

$$
a_{d}=-8 g_{s}^{2}-\frac{9}{4} g^{2}-\frac{5}{12} g^{\prime 2}, \quad a_{u}=a_{d}-g^{\prime 2},
$$

with $g_{s}, g$, and $g^{\prime}$ the gauge coupling constants of $S U(3)_{c}, S U(2)_{L}$, and $U(1)_{Y}$, respectively. Introducing

$$
\Gamma_{L}=\sum_{\rho=1}^{n=2} \Gamma_{\rho} \Gamma_{\rho}^{\dagger}, \quad \Gamma_{R}=\sum_{\rho=1}^{n=2} \Gamma_{\rho}^{\dagger} \Gamma_{\rho}, \quad \Delta_{L}=\sum_{\rho=1}^{n=2} \Delta_{\rho} \Delta_{\rho}^{\dagger}, \quad \text { and } \quad \Delta_{R}=\sum_{\rho=1}^{n=2} \Delta_{\rho}^{\dagger} \Delta_{\rho},
$$

Eqs. (39) and (40) read

$$
\begin{gathered}
\mathcal{D} \Gamma_{\alpha}=a_{d} \Gamma_{\alpha}+\sum_{\rho=1}^{n=2} T_{\alpha, \rho}^{d} \Gamma_{\rho}+\Gamma_{\alpha} \Gamma_{R}+\frac{1}{2} \Gamma_{L} \Gamma_{\alpha}+\frac{1}{2} \Delta_{L} \Gamma_{\alpha}-2 \sum_{\rho=1}^{n=2} \Delta_{\rho} \Delta_{\alpha}^{\dagger} \Gamma_{\rho}, \\
\mathcal{D} \Delta_{\alpha}=a_{u} \Delta_{\alpha}+\sum_{\rho=1}^{n=2} T_{\alpha, \rho}^{u} \Delta_{\rho}+\Delta_{\alpha} \Delta_{R}+\frac{1}{2} \Delta_{L} \Delta_{\alpha}+\frac{1}{2} \Gamma_{L} \Delta_{\alpha}-2 \sum_{\rho=1}^{n=2} \Gamma_{\rho} \Gamma_{\alpha}^{\dagger} \Delta_{\rho} .
\end{gathered}
$$

Equations (43) and (44) are the starting point to analyzing the one-loop stability of the necessary and sufficient conditions for $\mathrm{gFC}$. For that, one needs to know

$$
\mathcal{D}\left(\left[\Gamma_{\alpha} \Gamma_{\beta}^{\dagger}, \Gamma_{\gamma} \Gamma_{\delta}^{\dagger}\right]\right), \quad \mathcal{D}\left(\left[\Gamma_{\alpha}^{\dagger} \Gamma_{\beta}, \Gamma_{\gamma}^{\dagger} \Gamma_{\delta}\right]\right), \quad \mathcal{D}\left(\left[\Delta_{\alpha} \Delta_{\beta}^{\dagger}, \Delta_{\gamma} \Delta_{\delta}^{\dagger}\right]\right), \quad \mathcal{D}\left(\left[\Delta_{\alpha}^{\dagger} \Delta_{\beta}, \Delta_{\gamma}^{\dagger} \Delta_{\delta}\right]\right),
$$

under the assumption that Eq. (37) holds. With that objective in mind, some simplifications are worth mentioning. Starting with $\Gamma_{\alpha} \Gamma_{\beta}^{\dagger}$, we first notice that

$$
\mathcal{D}\left(\Gamma_{\alpha} \Gamma_{\beta}^{\dagger}\right)=\left(\mathcal{D} \Gamma_{\alpha}\right) \Gamma_{\beta}^{\dagger}+\Gamma_{\alpha}\left(\mathcal{D} \Gamma_{\beta}\right)^{\dagger}=f_{\alpha \beta}^{\left[\mathrm{d}_{\mathrm{L}}\right]}(\Gamma)+g_{\alpha \beta}^{\left[\mathrm{d}_{\mathrm{L}}\right]}(\Gamma, \Delta)
$$

with $^{2}$

$$
f_{\alpha \beta}^{\left[\mathrm{d}_{\mathrm{L}}\right]}(\Gamma)=2 a_{d} \Gamma_{\alpha} \Gamma_{\beta}^{\dagger}+\sum_{\rho=1}^{n=2}\left[T_{\alpha, \rho}^{d} \Gamma_{\rho} \Gamma_{\beta}^{\dagger}+T_{\beta, \rho}^{d *} \Gamma_{\alpha} \Gamma_{\rho}^{\dagger}\right]+2 \Gamma_{\alpha} \Gamma_{R} \Gamma_{\beta}^{\dagger}+\frac{1}{2} \Gamma_{L} \Gamma_{\alpha} \Gamma_{\beta}^{\dagger}+\frac{1}{2} \Gamma_{\alpha} \Gamma_{\beta}^{\dagger} \Gamma_{L},
$$

\footnotetext{
${ }^{2}$ The superscript $\left[\mathrm{d}_{\mathrm{L}}\right]$ is chosen in correspondence with the $\Gamma_{\alpha} \Gamma_{\beta}^{\dagger}$ matrix combinations; similarly, $f_{\alpha \beta}^{\left[\mathrm{d}_{\mathrm{R}}\right]}$ and $g_{\alpha \beta}^{\left[\mathrm{d}_{\mathrm{R}}\right]}$ will appear in $\mathcal{D}\left(\Gamma_{\alpha}^{\dagger} \Gamma_{\beta}\right)$, and $f_{\alpha \beta}^{\left[\mathrm{u}_{\mathrm{L}, \mathrm{R}}\right]}$ will appear in $\mathcal{D}\left(\Delta_{\alpha} \Delta_{\beta}^{\dagger}\right)$ and $\mathcal{D}\left(\Delta_{\alpha}^{\dagger} \Delta_{\beta}\right)$, but we concentrate for the moment on $\mathcal{D}\left(\Gamma_{\alpha} \Gamma_{\beta}^{\dagger}\right)$.
} 
and

$$
\begin{aligned}
g_{\alpha \beta}^{\left[\mathrm{d}_{\mathrm{L}}\right]}(\Gamma, \Delta)= & \frac{1}{2} \Delta_{L} \Gamma_{\alpha} \Gamma_{\beta}^{\dagger}+\frac{1}{2} \Gamma_{\alpha} \Gamma_{\beta}^{\dagger} \Delta_{L} \\
& -2 \sum_{\rho=1}^{n=2}\left[\Delta_{\rho} \Delta_{\alpha}^{\dagger} \Gamma_{\rho} \Gamma_{\beta}^{\dagger}+\Gamma_{\alpha} \Gamma_{\rho}^{\dagger} \Delta_{\beta} \Delta_{\rho}^{\dagger}\right]
\end{aligned}
$$

The relevant property of the decomposition in Eq. (46) is that $f_{\alpha \beta}^{\left[\mathrm{d}_{\mathrm{L}}\right]}$ depends only, ${ }^{3}$ in terms of matrices, on $\Gamma \Gamma^{\dagger}$ and $\Gamma \Gamma^{\dagger} \Gamma \Gamma^{\dagger}$ terms, while $g_{\alpha \beta}^{\left[\mathrm{d}_{\mathrm{L}}\right]}$ collects the remaining dependence on $\Delta^{\prime}$ 's, which has terms $\Gamma^{\dagger} \Delta \Delta^{\dagger}$ and $\Delta \Delta^{\dagger} \Gamma \Gamma^{\dagger}$. Then,

$$
\begin{aligned}
\mathcal{D}\left[\Gamma_{\alpha} \Gamma_{\beta}^{\dagger}, \Gamma_{\gamma} \Gamma_{\delta}^{\dagger}\right]= & {\left[\mathcal{D}\left(\Gamma_{\alpha} \Gamma_{\beta}^{\dagger}\right), \Gamma_{\gamma} \Gamma_{\delta}^{\dagger}\right]+\left[\Gamma_{\alpha} \Gamma_{\beta}^{\dagger}, \mathcal{D}\left(\Gamma_{\gamma} \Gamma_{\delta}^{\dagger}\right)\right] } \\
= & {\left[f_{\alpha \beta}^{\left[\mathrm{d}_{\mathrm{L}}\right]}(\Gamma)+g_{\alpha \beta}^{\left[\mathrm{d}_{\mathrm{L}}\right]}(\Gamma, \Delta), \Gamma_{\gamma} \Gamma_{\delta}^{\dagger}\right] } \\
& +\left[\Gamma_{\alpha} \Gamma_{\beta}^{\dagger}, f_{\gamma \delta}^{\left[\mathrm{d}_{\mathrm{L}}\right]}(\Gamma)+g_{\gamma \delta}^{\left[\mathrm{d}_{\mathrm{L}}\right]}(\Gamma, \Delta)\right] .
\end{aligned}
$$

\section{B. Evolution with gFC matrices}

It is clear that if there is gFC, i.e., with Eq. (37),

$$
\left[f_{\alpha \beta}^{\left[\mathrm{d}_{\mathrm{L}}\right]}(\Gamma), \Gamma_{\gamma} \Gamma_{\delta}^{\dagger}\right]=\left[\Gamma_{\alpha} \Gamma_{\beta}^{\dagger}, f_{\gamma \delta}^{\left[\mathrm{d}_{\mathrm{L}}\right]}(\Gamma)\right]=0,
$$

and thus

$$
\mathcal{D}\left[\Gamma_{\alpha} \Gamma_{\beta}^{\dagger}, \Gamma_{\gamma} \Gamma_{\delta}^{\dagger}\right]=\left[g_{\alpha \beta}^{\left[\mathrm{d}_{\alpha}\right]}(\Gamma, \Delta), \Gamma_{\gamma} \Gamma_{\delta}^{\dagger}\right]+\left[\Gamma_{\alpha} \Gamma_{\beta}^{\dagger}, g_{\gamma \delta}^{\left[\mathrm{d}_{\mathrm{L}}\right]}(\Gamma, \Delta)\right] .
$$

After the simplification brought by Eq. (50), the next step is to trade Eq. (51) for conditions expressed in terms of the physical parameters entering in the matrices $\mathcal{M}_{\mathrm{d}}^{0}, \mathcal{N}_{\mathrm{d}}^{0}, \mathcal{M}_{\mathrm{u}}^{0}$, and $\mathcal{N}_{\mathrm{u}}^{0}$. It is convenient to introduce the notation

$Y_{[\mathrm{d}] 1}^{0}=\mathcal{M}_{\mathrm{d}}^{0}, \quad Y_{[\mathrm{d}] 2}^{0}=\mathcal{N}_{\mathrm{d}}^{0}, \quad Y_{[\mathrm{u}] 1}^{0}=\mathcal{M}_{\mathrm{u}}^{0}, \quad Y_{[\mathrm{u}] 2}^{0}=\mathcal{N}_{\mathrm{u}}^{0}$,

which allows us to rewrite Eqs. (17) and (18) compactly (with summation over repeated indices understood),

$$
\begin{aligned}
\frac{v}{\sqrt{2}} \Gamma_{\alpha}=\mathrm{W}_{\alpha i} Y_{[\mathrm{d}] i}^{0}, & Y_{[\mathrm{d}] i}^{0}=\frac{v}{\sqrt{2}} \Gamma_{\alpha} \mathrm{W}_{\alpha i}^{*}, \\
\frac{v}{\sqrt{2}} \Delta_{\alpha}=\mathrm{W}_{\alpha i}^{*} Y_{[\mathrm{u}] i}^{0}, & Y_{[\mathrm{u}] i}^{0}=\frac{v}{\sqrt{2}} \Delta_{\alpha} \mathrm{W}_{\alpha i},
\end{aligned}
$$

where

$\mathrm{W}=\left(\begin{array}{cc}e^{-i \theta_{1}} & 0 \\ 0 & e^{-i \theta_{2}}\end{array}\right)\left(\begin{array}{cc}c_{\beta} & -s_{\beta} \\ s_{\beta} & c_{\beta}\end{array}\right), \quad \mathrm{WW}^{\dagger}=\mathrm{W}^{\dagger} \mathrm{W}=\mathbf{1}$.

For completeness, notice that

$$
\begin{array}{rlrl}
\frac{v}{\sqrt{2}} \Gamma_{\alpha}^{\dagger} & =\mathrm{W}_{\alpha i}^{*} Y_{[\mathrm{d}] i}^{0 \dagger}, & & \frac{v}{\sqrt{2}} \Delta_{\alpha}^{\dagger}=\mathrm{W}_{\alpha i} Y_{[\mathrm{u}]}^{0 \dagger}, \\
Y_{[\mathrm{d}] i}^{0 \dagger} & =\frac{v}{\sqrt{2}} \Gamma_{\alpha}^{\dagger} \mathrm{W}_{\alpha i}, & Y_{[\mathrm{u}] i}^{0^{\dagger}}=\frac{v}{\sqrt{2}} \Delta_{\alpha}^{\dagger} \mathrm{W}_{\alpha i}^{*} ;
\end{array}
$$

i.e., the Hermitian conjugate ${ }^{\dagger}$ (in the space of flavor indices) only gives a complex conjugate in $\mathrm{W}$. One can then write

$\left[\Gamma_{\alpha} \Gamma_{\beta}^{\dagger}, \Gamma_{\gamma} \Gamma_{\delta}^{\dagger}\right]=\frac{4}{v^{4}} \mathrm{~W}_{\alpha i} \mathrm{~W}_{\beta j}^{*} \mathrm{~W}_{\gamma k} \mathrm{~W}_{\delta l}^{*}\left[Y_{[\mathrm{d}] i}^{0} Y_{[\mathrm{d}] j}^{0 \dagger}, Y_{[\mathrm{d}] k}^{0} Y_{[\mathrm{d}] l]}^{0 \dagger}\right]$,

and thus

$$
\begin{aligned}
& \mathcal{D}\left[\Gamma_{\alpha} \Gamma_{\beta}^{\dagger}, \Gamma_{\gamma} \Gamma_{\delta}^{\dagger}\right] \\
&= \mathcal{D}\left(\frac{4}{v^{4}} \mathrm{~W}_{\alpha i} \mathrm{~W}_{\beta j}^{*} \mathrm{~W}_{\gamma k} \mathrm{~W}_{\delta l}^{*}\right)\left[Y_{[\mathrm{d}] i}^{0} Y_{[\mathrm{d}] j}^{0 \dagger}, Y_{[\mathrm{d}] k}^{0} Y_{[\mathrm{d}] l}^{0 \dagger}\right] \\
&+\frac{4}{v^{4}} \mathrm{~W}_{\alpha i} \mathrm{~W}_{\beta j}^{*} \mathrm{~W}_{\gamma k} \mathrm{~W}_{\delta l}^{*} \mathcal{D}\left[Y_{[\mathrm{d}]}^{0} Y_{[\mathrm{d}] j}^{0 \dagger}, Y_{[\mathrm{d}] k}^{0} Y_{[\mathrm{d}] l]}^{0 \dagger}\right] .
\end{aligned}
$$

With gFC, the first commutator vanishes, and we just have a linear combination of different $\mathcal{D}\left[Y_{[\mathrm{d}] i}^{0} Y_{[\mathrm{d}] j}^{0 \dagger}, Y_{[\mathrm{d}] k}^{0} Y_{[\mathrm{d}] l]}^{0 \dagger}\right]$. One can indeed invert Eq. (57),

$\frac{4}{v^{4}} \mathcal{D}\left[Y_{[\mathrm{d}] i}^{0} Y_{[\mathrm{d}] j}^{0 \dagger}, Y_{[\mathrm{d}] k}^{0} Y_{[\mathrm{d}] l}^{0 \dagger}\right]=\mathrm{W}_{\alpha i}^{*} \mathrm{~W}_{\beta j} \mathrm{~W}_{\gamma k}^{*} \mathrm{~W}_{\delta l} \mathcal{D}\left[\Gamma_{\alpha} \Gamma_{\beta}^{\dagger}, \Gamma_{\gamma} \Gamma_{\delta}^{\dagger}\right]$,

and express the right-hand side of Eq. (58) in terms of $Y_{[\mathrm{d}] i}^{0}$ and $Y_{[\mathrm{u}] j}^{0}$ :

$$
\begin{aligned}
& \frac{v^{2}}{2} \mathcal{D}\left[Y_{[\mathrm{d}] l}^{0} Y_{[\mathrm{d}] j}^{0 \dagger}, Y_{[\mathrm{d}] k}^{0} Y_{[\mathrm{d}] l}^{0 \dagger}\right]=Y_{[\mathrm{d}] i}^{0} Y_{[\mathrm{d}] j}^{0 \dagger} Y_{[\mathrm{u}] h}^{0} Y_{[\mathrm{u}] h}^{0 \dagger} Y_{[\mathrm{d}] k}^{0} Y_{[\mathrm{d}] l}^{0 \dagger}-Y_{[\mathrm{d}] k}^{0} Y_{[\mathrm{d}] l}^{0 \dagger} Y_{[\mathrm{u}] h}^{0} Y_{[\mathrm{u}] h}^{0 \dagger} Y_{[\mathrm{d}] i}^{0} Y_{[\mathrm{d}] j}^{0 \dagger} \\
& -2\left[Y_{[\mathrm{u}] h}^{0} Y_{[\mathrm{u}] i}^{0 \dagger}, Y_{[\mathrm{d}] k}^{0} Y_{[\mathrm{d}] l}^{0 \dagger}\right] Y_{[\mathrm{d}] h}^{0} Y_{[\mathrm{d}] j}^{0 \dagger}-2 Y_{[\mathrm{d}] i}^{0} Y_{[\mathrm{d}] h}^{0 \dagger}\left[Y_{[\mathrm{u}] j}^{0} Y_{[\mathrm{u}] h}^{0 \dagger}, Y_{[\mathrm{d}] k}^{0} Y_{[\mathrm{d}] l}^{0 \dagger}\right] \\
& +2\left[Y_{[\mathrm{u}] h}^{0} Y_{[\mathrm{u}] k}^{0 \dagger}, Y_{[\mathrm{d}] i}^{0} Y_{[\mathrm{d}] j}^{0 \dagger}\right] Y_{[\mathrm{d}] h}^{0} Y_{[\mathrm{d}] l}^{0 \dagger}+2 Y_{[\mathrm{d}] k}^{0} Y_{[\mathrm{d}] h}^{0 \dagger}\left[Y_{[\mathrm{u}] l}^{0} Y_{[\mathrm{u}] h}^{0 \dagger}, Y_{[\mathrm{d}] l}^{0} Y_{[\mathrm{d}] j}^{0 \dagger}\right] .
\end{aligned}
$$

\footnotetext{
${ }^{3}$ Although $T_{\alpha, \rho}^{d}$ do depend on $\Delta_{\alpha}$ 's, there is no matrix dependence, only $\mathbb{C}$ numbers; this also applies to the leptonic Yukawa couplings $\Pi_{\alpha}$.
} 
As expected from the discussion in Sec. II C, having a gFC scenario is related to the Yukawa coupling matrices themselves; it does not hinge on the particular electroweak vacuum configuration that determines which particular combinations of them are the mass matrices $\mathcal{M}_{\mathrm{d}}^{0}$ and $\mathcal{M}_{\mathrm{u}}^{0}$ and the matrices $\mathcal{N}_{\mathrm{d}}^{0}$ and $\mathcal{N}_{\mathrm{u}}^{0}$ [the vacuum configuration is "encoded" in $\mathrm{W}$, which does not appear in Eq. (59)]. The last step is to transform into the mass eigenstate basis with $U_{\mathrm{d}_{L}}$ in Eq. (19),

$$
\begin{aligned}
\frac{v^{2}}{2} U_{\mathrm{d}_{L}}^{\dagger}\left(\mathcal{D}\left[Y_{[\mathrm{d}]}^{0} Y_{[\mathrm{d}] j}^{0 \dagger}, Y_{[\mathrm{d}] k}^{0} Y_{[\mathrm{d}] l}^{0 \dagger}\right]\right) U_{\mathrm{d} L}= & Y_{[\mathrm{d}] i} Y_{[\mathrm{d}] j}^{\dagger} V^{\dagger} Y_{[\mathrm{u}] h} Y_{[\mathrm{u}] h}^{\dagger} V Y_{[\mathrm{d}] k} Y_{[\mathrm{d}] l}^{\dagger}-Y_{[\mathrm{d}] k} Y_{[\mathrm{d}] l}^{\dagger} V^{\dagger} Y_{[\mathrm{u}] h} Y_{[\mathrm{u}] h}^{\dagger} V Y_{[\mathrm{d}] i} Y_{[\mathrm{d}] j}^{\dagger} \\
& -2\left[V^{\dagger} Y_{[\mathrm{u}] h} Y_{[\mathrm{u}]}^{\dagger} V, Y_{[\mathrm{d}] k} Y_{[\mathrm{d}] l}^{\dagger}\right] Y_{[\mathrm{d}] h} Y_{[\mathrm{d}] j}^{\dagger}-2 Y_{[\mathrm{d}]} Y_{[\mathrm{d}] h}^{\dagger}\left[V^{\dagger} Y_{[\mathrm{u}] j} Y_{[\mathrm{u}] h}^{\dagger} V, Y_{[\mathrm{d}] k} Y_{[\mathrm{d}] l}^{\dagger}\right] \\
& +2\left[V^{\dagger} Y_{[\mathrm{u}] h} Y_{[\mathrm{u}] k}^{\dagger} V, Y_{[\mathrm{d}] i} Y_{[\mathrm{d}] j}^{\dagger}\right] Y_{[\mathrm{d}] h} Y_{[\mathrm{d}] l}^{\dagger}+2 Y_{[\mathrm{d}] k} Y_{[\mathrm{d}] h}^{\dagger}\left[V^{\dagger} Y_{[\mathrm{u}] l} Y_{[\mathrm{u}] h}^{\dagger} V, Y_{[\mathrm{d}] i} Y_{[\mathrm{d}] j}^{\dagger}\right],
\end{aligned}
$$

where the CKM matrix $V=U_{\mathrm{u}_{L}}^{\dagger} U_{\mathrm{d}_{L}}$ appears together with the diagonal matrices

$$
Y_{[\mathrm{d}] 1}=\mathcal{M}_{\mathrm{d}}, \quad Y_{[\mathrm{d}] 2}=\mathcal{N}_{\mathrm{d}}, \quad Y_{[\mathrm{u}] 1}=\mathcal{M}_{\mathrm{u}}, \quad Y_{[\mathrm{u}] 2}=\mathcal{N}_{\mathrm{u}}
$$

In this generic notation [Eq. (52)],

$$
\begin{aligned}
Y_{[\mathrm{d}] i}=\operatorname{diag}\left(y_{i, j}^{\mathrm{d}}\right), & \left\{y_{1,1}^{\mathrm{d}}, y_{1,2}^{\mathrm{d}}, y_{1,3}^{\mathrm{d}}\right\}=\left\{m_{d}, m_{s}, m_{b}\right\}, \\
& \left\{y_{2,1}^{\mathrm{d}}, y_{2,2}^{\mathrm{d}}, y_{2,3}^{\mathrm{d}}\right\}=\left\{n_{d}, n_{s}, n_{b}\right\}, \\
Y_{[\mathrm{u}] i}=\operatorname{diag}\left(y_{i, j}^{\mathrm{u}}\right), & \left\{y_{1,1}^{\mathrm{u}}, y_{1,2}^{\mathrm{u}}, y_{1,3}^{\mathrm{u}}\right\}=\left\{m_{u}, m_{c}, m_{t}\right\}, \\
& \left\{y_{2,1}^{\mathrm{u}}, y_{2,2}^{\mathrm{u}}, y_{2,3}^{\mathrm{u}}\right\}=\left\{n_{u}, n_{c}, n_{t}\right\} .
\end{aligned}
$$

The previous derivation concerns the set $\left\{\Gamma_{\alpha} \Gamma_{\beta}^{\dagger}\right\}$; the evolution equations for $\left\{\Gamma_{\alpha}^{\dagger} \Gamma_{\beta}\right\},\left\{\Delta_{\alpha} \Delta_{\beta}^{\dagger}\right\}$ and $\left\{\Delta_{\alpha}^{\dagger} \Delta_{\beta}\right\}$ are given in the Appendix.

To have a $\mathrm{gFC}$ scenario stable under the one-loop RGE, one needs the simultaneous diagonalizability of $\left\{Y_{[\mathrm{q}] 1}^{0}, Y_{[\mathrm{q}] 2}^{0}\right\}$ to be preserved, that is,

$\mathcal{D}\left[Y_{[\mathrm{d}] i}^{0} Y_{[\mathrm{d}] j}^{0 \dagger}, Y_{[\mathrm{d}] k}^{0} Y_{[\mathrm{d}] l}^{0 \dagger}\right]=0, \quad \mathcal{D}\left[Y_{[\mathrm{u}] i}^{0} Y_{[\mathrm{u}] j}^{0 \dagger}, Y_{[\mathrm{u}] k}^{0} Y_{[\mathrm{u}] l}^{0 \dagger}\right]=0$, $\mathcal{D}\left[Y_{[\mathrm{d}] i}^{0 \dagger} Y_{[\mathrm{d}] j}^{0}, Y_{[\mathrm{d}] k}^{0 \dagger} Y_{[\mathrm{d}]}^{0}\right]=0, \quad \mathcal{D}\left[Y_{[\mathrm{u}] i}^{0 \dagger} Y_{[\mathrm{u}] j}^{0}, Y_{[\mathrm{u}] k}^{0 \dagger} Y_{[\mathrm{u}] l}^{0}\right]=0$.

With Eqs. (60)-(62), the conditions expressed by the matrix equations in (63) are formulated in full generality, for fixed mass matrices $\mathcal{M}_{\mathrm{d}}$ and $\mathcal{M}_{\mathrm{u}}$ and CKM mixings $V$, in terms of the six complex parameters $n_{j}$ in Eq. (38). For example, element $(a, b)$ of the first stability condition in Eq. (63), for $i=j, k=l, \mathcal{D}\left[Y_{[\mathrm{d}] i}^{0} Y_{[\mathrm{d}] i}^{0 \dagger}, Y_{[\mathrm{d}] k}^{0} Y_{[\mathrm{d}] k}^{0^{\dagger}}\right]=0$, reads

$$
\begin{aligned}
0= & \sum_{q=1}^{3} \sum_{h=1}^{2} V_{q a}^{*} V_{q b}\left\{\left|y_{h, q}^{\mathrm{u}}\right|^{2}\left(\left|y_{i, a}^{\mathrm{d}}\right|^{2}\left|y_{k, b}^{\mathrm{d}}\right|^{2}-\left|y_{i, b}^{\mathrm{d}}\right|^{2}\left|y_{k, a}^{\mathrm{d}}\right|^{2}\right)\right. \\
& -2\left(y_{h, q}^{\mathrm{u}} y_{i, q}^{\mathrm{u} *} y_{h, b}^{\mathrm{d}} y_{i, b}^{\mathrm{d} *}+y_{i, q}^{\mathrm{u}} y_{h, q}^{\mathrm{u} *} y_{i, a}^{\mathrm{d}} y_{h, a}^{\mathrm{d} *}\right)\left(\left|y_{k, b}^{\mathrm{d}}\right|^{2}-\left|y_{k, a}^{\mathrm{d}}\right|^{2}\right) \\
& \left.+2\left(y_{h, q}^{\mathrm{u}} y_{k, q}^{\mathrm{u} *} y_{h, b}^{\mathrm{d}} y_{k, b}^{\mathrm{d} *}+y_{k, q}^{\mathrm{u}} y_{h, q}^{\mathrm{u} *} y_{k, a}^{\mathrm{d}} y_{h, a}^{\mathrm{d} *}\right)\left(\left|y_{i, b}^{\mathrm{d}}\right|^{2}-\left|y_{i, a}^{\mathrm{d}}\right|^{2}\right)\right\} .
\end{aligned}
$$

The complete set of conditions is given in the Appendix. For each set in Eq. (63), there are six choices of $i, j, k$, $l=1,2$, in the 2HDM, which give, at least, three independent complex equations each. It is clear that, in terms of the six complex parameters $n_{j}$, the system is largely overconstrained. In Sec. III C below, we check that the known stable solutions with $\mathcal{N}_{\mathrm{f}} \propto \mathcal{M}_{\mathrm{f}}$ are recovered. It is, however, beyond the scope of this work to address if other solutions could a priori exist for the general one-loop RGE stability conditions of gFC.

The lepton sector is discussed in Sec. III D. Finally, in Sec. III E, we present some particular solutions that arise when the CKM matrix reduces to a Cabibbo-like blockdiagonal mixing.

\section{Stable gFC with $\mathcal{N}_{\mathrm{f}} \propto \mathcal{M}_{\mathrm{f}}$}

When one substitutes $\mathcal{N}_{\mathrm{q}}=\alpha_{q} \mathcal{M}_{\mathrm{q}}, \alpha_{q} \in \mathbb{C}$, in the conditions for one-loop RGE stability of $\mathrm{gFC}$ given in the Appendix, solving them for $\alpha_{u}$ and $\alpha_{d}$ reduces to finding solutions of

$$
\left(1+\alpha_{d} \alpha_{u}\right)\left(\alpha_{u}^{*}-\alpha_{d}\right)=0,
$$

that is, $\alpha_{u}=-\alpha_{d}^{-1}$ or $\alpha_{u}^{*}=\alpha_{d}$. In both cases, there is a basis for the scalars [77]

$$
\left(\begin{array}{l}
H_{1}^{\prime} \\
H_{2}^{\prime}
\end{array}\right)=\frac{1}{\sqrt{1+\left|\alpha_{d}\right|^{2}}}\left(\begin{array}{cc}
1 & \alpha_{d} \\
-\alpha_{d}^{*} & 1
\end{array}\right)\left(\begin{array}{l}
H_{1} \\
H_{2}
\end{array}\right),
$$

with $H_{1}$ and $H_{2}$ in Eq. (3), such that in Eq. (11) $H_{1}^{\prime}$ couples only to $d_{R}^{0}$ while $H_{2}^{\prime}$ couples only to $u_{R}^{0}$ for $\alpha_{u}=-\alpha_{d}^{-1}$; for $\alpha_{u}=\alpha_{d}^{*}, H_{1}^{\prime}$ couples to $d_{R}^{0}$ and $u_{R}^{0}$, while $H_{2}^{\prime}$ does not. These cases are none other than the 2HDMs of types II and I, respectively. For the particular case $\alpha_{u}=\alpha_{d}^{*}=0$, the scalar doublet that has a zero vacuum expectation value has vanishing Yukawa couplings: this is the inert 2HDM [29]. 


\section{Stable gFC in the lepton sector}

The one-loop RGE of the lepton Yukawa couplings in Eq. (29) reads $[87,88]$

$$
\begin{gathered}
\mathcal{D} \Pi_{\alpha}=a_{\ell} \Pi_{\alpha}+\sum_{\rho=1}^{n=2} T_{\alpha, \rho}^{\ell} \Pi_{\rho}+\sum_{\rho=1}^{n=2}\left(\Pi_{\alpha} \Pi_{\rho}^{\dagger} \Pi_{\rho}+\frac{1}{2} \Pi_{\rho} \Pi_{\rho}^{\dagger} \Pi_{\alpha}\right) \\
\text { with } T_{\alpha, \rho}^{\ell}=T_{\alpha, \rho}^{d},
\end{gathered}
$$

where $\quad a_{\ell}=-\frac{9}{4} g^{2}-\frac{15}{4} g^{2}$. With $\quad \Pi_{L}=\sum_{\rho=1}^{n=2} \Pi_{\rho} \Pi_{\rho}^{\dagger}$, $\Pi_{R}=\sum_{\rho=1}^{n=2} \Pi_{\rho}^{\dagger} \Pi_{\rho}$,

$$
\mathcal{D} \Pi_{\alpha}=a_{\ell} \Pi_{\alpha}+\sum_{\rho=1}^{n=2} T_{\alpha, \rho}^{\ell} \Pi_{\rho}+\Pi_{\alpha} \Pi_{R}+\frac{1}{2} \Pi_{L} \Pi_{\alpha} .
$$

The crucial difference in the leptonic sector is that, following Eq. (68),

$$
\begin{aligned}
\mathcal{D}\left(\Pi_{\alpha} \Pi_{\beta}^{\dagger}\right)= & 2 a_{\ell} \Pi_{\alpha} \Pi_{\beta}^{\dagger} \\
& +\sum_{\rho=1}^{n=2}\left(T_{\alpha, \rho}^{\ell} \Pi_{\rho} \Pi_{\beta}^{\dagger}+T_{\beta, \rho}^{\ell *} \Pi_{\alpha} \Pi_{\rho}^{\dagger}\right)+2 \Pi_{\alpha} \Pi_{R} \Pi_{\beta}^{\dagger} \\
& +\frac{1}{2}\left(\Pi_{L} \Pi_{\alpha} \Pi_{\beta}^{\dagger}+\Pi_{\alpha} \Pi_{\beta}^{\dagger} \Pi_{L}\right)
\end{aligned}
$$

and thus it is clear that if $\left\{\Pi_{\alpha} \Pi_{\beta}^{\dagger}\right\}_{\alpha, \beta=1,2}$ is Abelian then

$$
\begin{aligned}
\mathcal{D}\left[\Pi_{\alpha} \Pi_{\beta}^{\dagger}, \Pi_{\gamma} \Pi_{\delta}^{\dagger}\right]= & {\left[\mathcal{D}\left(\Pi_{\alpha} \Pi_{\beta}^{\dagger}\right), \Pi_{\gamma} \Pi_{\delta}^{\dagger}\right] } \\
& +\left[\Pi_{\alpha} \Pi_{\beta}^{\dagger}, \mathcal{D}\left(\Pi_{\gamma} \Pi_{\delta}^{\dagger}\right)\right]=0 .
\end{aligned}
$$

Similarly,

$$
\begin{aligned}
\mathcal{D}\left(\Pi_{\alpha}^{\dagger} \Pi_{\beta}\right)= & 2 a_{\ell} \Pi_{\alpha}^{\dagger} \Pi_{\beta}+\sum_{\rho=1}^{n=2}\left(T_{\alpha, \rho}^{\ell *} \Pi_{\rho}^{\dagger} \Pi_{\beta}+T_{\beta, \rho}^{\ell} \Pi_{\alpha}^{\dagger} \Pi_{\rho}\right) \\
& +\Pi_{R} \Pi_{\alpha}^{\dagger} \Pi_{\beta}^{\dagger}+\Pi_{\alpha}^{\dagger} \Pi_{\beta}^{\dagger} \Pi_{R}+\Pi_{\alpha}^{\dagger} \Pi_{L} \Pi_{\beta},
\end{aligned}
$$

and thus, if $\left\{\Pi_{\alpha}^{\dagger} \Pi_{\beta}\right\}_{\alpha, \beta=1,2}$ is Abelian, then

$$
\begin{aligned}
\mathcal{D}\left[\Pi_{\alpha}^{\dagger} \Pi_{\beta}, \Pi_{\gamma}^{\dagger} \Pi_{\delta}\right]= & {\left[\mathcal{D}\left(\Pi_{\alpha}^{\dagger} \Pi_{\beta}\right), \Pi_{\gamma}^{\dagger} \Pi_{\delta}\right] } \\
& +\left[\Pi_{\alpha}^{\dagger} \Pi_{\beta}, \mathcal{D}\left(\Pi_{\gamma}^{\dagger} \Pi_{\delta}\right)\right]=0 .
\end{aligned}
$$

That is, if the Yukawa couplings of leptons are gFC, as in Eq. (38), this is not altered by the RGE: general flavor alignment is one-loop stable in the lepton sector. This can be directly traced back to the absence of right-handed neutrinos and Yukawa couplings involving them in Eq. (29), in clear contrast with the quark sector. This result represents a generalization of previous results restricted to the so-called aligned case and pointed out in Ref. [79], in agreement with the findings of Refs. [82,89]: at one loop level the charged lepton sector remains general Flavour
Conserving in full generality without any additional constraint. To be specific and going to the simplest aligned cases, types I, II, X, and Y models are defined in the quark sector by

$$
\begin{aligned}
& \text { Type I, X }\left\{\begin{array}{l}
\mathcal{N}_{\mathrm{d}}=\cot \beta \mathcal{M}_{\mathrm{d}}, \\
\mathcal{N}_{\mathrm{u}}=\cot \beta \mathcal{M}_{\mathrm{u}},
\end{array}\right. \\
& \text { Type I, Y }\left\{\begin{array}{l}
\mathcal{N}_{\mathrm{d}}=-\tan \beta \mathcal{M}_{\mathrm{d}}, \\
\mathcal{N}_{\mathrm{u}}=\cot \beta \mathcal{M}_{\mathrm{u}} .
\end{array}\right.
\end{aligned}
$$

The fact that the leptonic sector alignment was known to be stable under RGE implies that one could analyze the experimental data with the previous equation together with the more general leptonic structure $\left(\Pi_{2}=\xi_{\ell} e^{-i \theta} \Pi_{1}\right)$

$$
\mathcal{N}_{\ell}=\cot \beta\left(\frac{-\tan \beta+\xi_{\ell}}{\cot \beta+\xi_{\ell}}\right) \mathcal{M}_{\ell}
$$

in the framework of a model one-loop stable under RGE. This would include in a single analysis both types I and X or types II and Y. Note that with the appropriate limit $\xi_{\ell} \rightarrow$ 0 or $\xi_{\ell} \rightarrow \infty$ one recovers the four models. Equation (72) implies the new more general result that the models implemented by Eq. (73) together with an arbitrary diagonal $\mathcal{N}_{\ell}$ [not just with Eq. (74)] are one-loop stable under RGE.

\section{E. Stable gFC with Cabibbo-like mixing}

The CKM matrix has a hierarchical structure; keeping only the largest mixing, it has the form

$$
V_{\theta_{c}}=\left(\begin{array}{ccc}
\cos \theta_{c} & \sin \theta_{c} & 0 \\
-\sin \theta_{c} & \cos \theta_{c} & 0 \\
0 & 0 & 1
\end{array}\right)
$$

with $\theta_{c} \simeq 0.22$ the Cabibbo mixing angle. It is interesting to analyze the question of one-loop RGE stability of $\mathrm{gFC}$ conditions with $V \rightarrow V_{\theta_{c}}$ in Eq. (75). First, it is interesting on its own to know if this simplified mixing allows for some stable gFC scenario; second, if that is the case, in terms of those $\mathcal{N}_{\mathrm{q}}$ matrices, the deviations of $\mathrm{gFC}$ produced by the RGE would be controlled by the initial deviations of the complete CKM matrix from $V_{\theta_{c}}$, the subleading mixings.

One should first notice that, since $V_{\theta_{c}}$ decouples the third quark generation, $n_{b}$ and $n_{t}$ are expected to remain free parameters. Then, since the only remaining stability conditions concern elements $(a, b)=(1,2)$ or $(2,1)$, all the mixing combinations $V_{q a}^{*} V_{q b}, V_{a q} V_{b q}^{*}$ equal either $\cos \theta_{c} \sin \theta_{c}$ or $-\cos \theta_{c} \sin \theta_{c}$, and thus the dependence of the stability conditions on $\theta_{c}$ disappears.

Two classes of stable $\mathrm{gFC}$ scenarios follow from the discussion in Sec. III C. The first, with 


$$
\begin{aligned}
& \mathcal{N}_{\mathrm{d}}=\operatorname{diag}\left(\alpha m_{d}, \alpha m_{s}, n_{b}\right), \\
& \mathcal{N}_{\mathrm{u}}=\operatorname{diag}\left(\alpha^{*} m_{u}, \alpha^{*} m_{c}, n_{t}\right),
\end{aligned}
$$

corresponds to a type-I 2HDM for the first two generations, while $n_{b}$ and $n_{t}$ are free (and thus $\mathcal{M}_{\mathrm{q}}^{-1} \mathcal{N}_{\mathrm{q}} \neq \alpha_{q} \mathbf{1}$ ). Some particular limit - the extreme chiral limit—of Eq. (76) was already obtained in Ref. [79] to justify $V \simeq \mathbf{1}$. The second is

$$
\begin{aligned}
& \mathcal{N}_{\mathrm{d}}=\operatorname{diag}\left(\alpha m_{d}, \alpha m_{s}, n_{b}\right), \\
& \mathcal{N}_{\mathrm{u}}=\operatorname{diag}\left(-\alpha^{-1} m_{u},-\alpha^{-1} m_{c}, n_{t}\right),
\end{aligned}
$$

which corresponds instead to a type-II $2 \mathrm{HDM}$ for the first two generations (with free $n_{b}, n_{t}$, and $\mathcal{M}_{\mathrm{q}}^{-1} \mathcal{N}_{\mathrm{q}} \neq \alpha_{q} \mathbf{1}$, too). In addition to Eqs. (76) and (77), one can check that

$$
\begin{aligned}
& \mathcal{N}_{\mathrm{d}}=\operatorname{diag}\left(e^{i \varphi_{d}} m_{s}, e^{i \varphi_{d}} m_{d}, n_{b}\right), \\
& \mathcal{N}_{\mathrm{u}}=\operatorname{diag}\left(e^{i \varphi_{u}} m_{c}, e^{i \varphi_{u}} m_{u}, n_{t}\right),
\end{aligned}
$$

with arbitrary real $\varphi_{d}$ and $\varphi_{u}$ (and again, arbitrary complex $n_{b}$ and $n_{t}$ ), gives indeed another stable gFC scenario in which $\mathcal{N}_{\mathrm{q}}$ and $\mathcal{M}_{\mathrm{q}}$ are not even proportional in the first two generations sector.

\section{PHENOMENOLOGY}

\section{A. General considerations}

The Yukawa interactions in Eq. (25) or (28), together with the absence of tree-level FCNCs parametrized in Eq. (38), have interesting phenomenological consequences in different observables, since they may produce deviations from SM expectations. Those windows on new physics in different observables are, of course, related: they are controlled by the parameters $n_{j}$ in Eq. (38), by the values of the masses $m_{\mathrm{H}^{ \pm}}, m_{\mathrm{H}}$, and $m_{\mathrm{A}}$ and by the mixings in the scalar sector, $\mathcal{R}_{i j}$ in Eq. (25). In the following, we consider for simplicity the $C P$-conserving case in Eq. (27). Our interest lies in the parameters $n_{j}$ in Eq. (38). Among the observables of interest, those that (i) involve the lowest number of new non-SM parameters and (ii) provide direct constraints from existing measurements are the following:

(i) Observables probing the couplings of the $125 \mathrm{GeV}$ Higgs-like scalar, which we identify with h, that is, (i) production mechanisms and (ii) decay modes. In addition to the $n_{j}$ parameters, they involve one extra parameter, the mixing $c_{\beta \alpha}$ if there is no $C P$ violation in the scalar sector; in the general case, two independent mixings are involved.

(ii) Observables probing the couplings of the charged scalar $\mathrm{H}^{ \pm}$, in particular, effects of $\mathrm{H}^{ \pm}$in flavorchanging processes in which the SM contributions involve virtual $W^{ \pm}$exchange like (i) tree-level decays, modifying, e.g., the expected universality of weak interactions, and (ii) one-loop FCNC processes like neutral meson mixings and rare decays. These observables, besides the $n_{j}$ parameters, depend on the mass $m_{\mathrm{H}^{ \pm}}$(and no dependence on the neutral scalar mixings).

We concentrate in the rest of this work on the flavorconserving observables related to $\mathrm{h}$ : besides probing the gFC matrices in Eq. (38), the bounds they impose also apply to the same flavor-conserving couplings of a general 2HDM. As mentioned above, we should stress that, in this context, there are other relevant constraints and that, in general, they can have an important impact on the model considered. Among them, one can include the oblique $S, T$, and $U$ parameters, flavor physics observables in FCNC processes like the radiative $b \rightarrow s \gamma$ decay, rare decays like $B_{s} \rightarrow \mu^{+} \mu^{-}$. or the neutral meson mixings; all these processes are one-loop induced in the $\mathrm{gFC}$ scenario and therefore depend on the masses of the new scalars. Treelevel processes like $B^{+} \rightarrow \tau^{+} \nu_{\tau}$ also depend on the new scalar masses. Other constraints depend also on the details of the scalar potential, like the stability and unitarity requirements on the Higgs sector. In the present analysis, we are interested in the new parameters introduced in the $\mathrm{gFC}$ case, so we will not consider all these constraints that should have an additional impact on the Higgs potential parameters.

Before addressing the different constraints related to experiment, one can formulate a first theoretical requirement on the perturbativity of the Yukawa couplings:

$$
\frac{\left|n_{j}\right|}{v} \leq \mathcal{O}(1) .
$$

The precise value adopted in Eq. (79), e.g., $\mathcal{O}(1) \rightarrow 1$ or $\sqrt{4 \pi}$, is not expected to be specially relevant; other phenomenological requirements will be, typically, more restrictive. There is, however, an exception: the "decoupling limit" [90] of the 2HDM, in which $s_{\beta \alpha} \rightarrow 1\left(c_{\beta \alpha} \rightarrow 0\right)$ removes the non-SM effects from the $\mathrm{h}$ couplings (while $m_{\mathrm{H}^{ \pm}} \gg v$ suppresses $\mathrm{H}^{ \pm}$-mediated non-SM effects), leaving the perturbativity requirement as the only effective constraint. One may further argue that having either $m_{j} \ll\left|n_{j}\right|$ or $m_{j} \gg\left|n_{j}\right|$ involves fine-tuning between quantities of very different nature: both $m_{j}$ and $n_{j}$ are linear combinations, controlled by $\beta$, of Yukawa couplings (times $v$ ), but $\beta$ originates in the scalar potential, meaning that very disparate values of $m_{j}$ and $n_{j}$ involve significant cancellations in one or the other, unless $\beta \rightarrow 0$ or $\beta \rightarrow \pi / 2$. For the sake of clarity, we will only consider Eq. (79) and ignore the previous concerns about eventual fine-tuning.

\section{B. Production and decay of $\mathbf{h}$}

For the observables related to $\mathrm{h}$, one should consider constraints on $n_{j}$ and $c_{\beta \alpha}$ arising from $\mathrm{h}$ production and decay processes at the LHC [91]. In connection to them, additional attention should be paid to the decays of $h$ into 
light fermions since enhanced decays into light fermions can increase the total width and modify the precise SM pattern of branching ratios. The cross sections for direct $q \bar{q} \rightarrow \mathrm{h}$ production are also important, since large couplings of $\mathrm{h}$ to light quarks, in combination with the luminosities given by the parton distribution functions, could significantly increase them.

Before addressing the Yukawa couplings themselves, we recall that, owing to the mixing in the scalar sector, the couplings $\mathrm{h} V V(V=W, Z)$ are modified with respect to the $\mathrm{SM}$ as

$$
\mathrm{h} V V, \quad \mathrm{SM}: m_{V} \mapsto \mathrm{gFC}-2 \mathrm{HDM}: s_{\beta \alpha} m_{V} .
$$

These couplings are involved in vector boson fusion (VBF) and associated production mechanisms and in decays $\mathrm{h} \rightarrow V V^{*}$.

For the different couplings to fermions $\mathcal{L}_{\mathrm{h} f f}=$ $-\mathrm{h} \bar{f}\left(a_{f}+i b_{f} \gamma_{5}\right) f$ in Eq. (28), we have a scalar term $a_{f}$, straightforward to compare with the SM one,

$a_{f}: \mathrm{SM}: m_{j} / v \mapsto \mathrm{gFC}-2 \mathrm{HDM}:\left(s_{\beta \alpha} m_{j}+c_{\beta \alpha} \operatorname{Re}\left(n_{j}\right)\right) / v$,

$$
\Gamma(\mathrm{h} \rightarrow \bar{f} f)_{\mathrm{SM}}: \frac{N_{c}(f)}{8 \pi} \frac{m_{\mathrm{h}}}{v^{2}} m_{f}^{2} \mapsto \Gamma(\mathrm{h} \rightarrow \bar{f} f)_{\mathrm{gFC}-2 \mathrm{HDM}}:
$$

The decay $\mathrm{h} \rightarrow \gamma \gamma$, central in the discovery of the Higgs, has an amplitude controlled in the SM by two interfering contributions, the one-loop triangle diagrams with virtual $W$ 's and top quarks. The former is modified according to Eq. (80). The latter is the only relevant one involving quarks in the SM because of the large h $\bar{t} t$ coupling: $m_{t} / v$; this amplitude is modified according to Eq. (81). With a pseudoscalar coupling now present, Eq. (82), there is an additional contribution that, however, does not interfere with the SM-like top(scalar coupling) $+W$. Furthermore, there are other contributions that one may consider: one due to diagrams with virtual $\mathrm{H}^{ \pm}$'s and the ones due to other fermions with enhanced couplings to h due to sizable $n_{j}$. and a pseudoscalar term $b_{f}$ absent in the SM,

$$
b_{f}: \mathrm{SM}: 0 \mapsto \mathrm{gFC}-2 \mathrm{HDM}: c_{\beta \alpha} \operatorname{Im}\left(n_{j}\right) / v .
$$

We now discuss in turn decay and production processes.

\section{Decays of $h$}

The decay width $\mathrm{h} \rightarrow \bar{f} f$, for a generic Yukawa interaction $\mathcal{L}_{\mathrm{h} f f}=-\mathrm{h} \bar{f}\left(a_{f}+i b_{f} \gamma_{5}\right) f$, is, at tree level,

$\Gamma(\mathrm{h} \rightarrow \bar{f} f)=N_{c}(f) \frac{m_{\mathrm{h}}}{8 \pi} \sqrt{1-4 \frac{m_{f}^{2}}{m_{\mathrm{h}}^{2}}}\left[\left(1-4 \frac{m_{f}^{2}}{m_{\mathrm{h}}^{2}}\right)\left|a_{f}\right|^{2}+\left|b_{f}\right|^{2}\right]$,

with $N_{c}=3$ for quarks and $N_{c}=1$ for leptons; neglecting $4 m_{f}^{2} / m_{\mathrm{h}}^{2} \ll 1$,

$$
\Gamma(\mathrm{h} \rightarrow \bar{f} f)=N_{c}(f) \frac{m_{\mathrm{h}}}{8 \pi}\left[\left|a_{f}\right|^{2}+\left|b_{f}\right|^{2}\right] .
$$

With Eqs. (81) and (82),

$\frac{N_{c}(f)}{8 \pi} \frac{m_{\mathrm{h}}}{v^{2}}\left[s_{\beta \alpha}^{2} m_{f}^{2}+2 s_{\beta \alpha} c_{\beta \alpha} m_{f} \operatorname{Re}\left(n_{f}\right)+c_{\beta \alpha}^{2}\left|n_{f}\right|^{2}\right]$.

For the charged scalar, they cannot be neglected if $\mathrm{H}^{ \pm}$is relatively light, and thus, barring that possibility, we do not consider them. For the remaining fermions, the values of $c_{\beta \alpha}$ that $\mathrm{h} \leftrightarrows W W$ decay and production require are typically small $\left(\left|c_{\beta \alpha}\right| \leq 0.1\right)$, and thus the values of $n_{j}$ that one would need for their contributions to be relevant would be at least $n_{j} \sim m_{t}$ : they would produce huge contributions to the width $\Gamma(\mathrm{h})$ or to $\bar{q} q \rightarrow \mathrm{h}$ production cross sections (see the discussion in Sec. IV B 2), in addition to the perturbativity and fine-tuning concerns on the Yukawa couplings already mentioned, and we thus ignore them altogether, since they will be rendered negligible once other constraints are considered. The width of $h \rightarrow \gamma \gamma$ reads

$$
\begin{aligned}
\Gamma(\mathrm{h} & \rightarrow \gamma \gamma)_{\mathrm{gFC}-2 \mathrm{HDM}} \\
& =\frac{\alpha^{2}}{256 \pi^{3}} \frac{m_{\mathrm{h}}^{3}}{v^{2}}\left(\left|\sum_{f} N_{c}(f) Q_{f}^{2} \frac{a_{f} v}{m_{f}} A_{F}\left(x_{f}\right)+s_{\beta \alpha} A_{V}\left(x_{W}\right)+g_{\mathrm{H}^{ \pm}} A_{S}\left(x_{\mathrm{H}^{ \pm}}\right)\right|^{2}+\left|\sum_{f} N_{c}(f) Q_{f}^{2} \frac{b_{f} v}{m_{f}} \hat{A}_{F}\left(x_{f}\right)\right|^{2}\right),
\end{aligned}
$$

with $x_{X}=4 m_{X}^{2} / m_{\mathrm{h}}^{2}$. The sum over fermions $f$ includes up- and down-type quarks, with $Q_{f}=2 / 3$ and $-1 / 3$, respectively, and charged leptons with $Q_{f}=-1$. The contribution of the charged scalar $\mathrm{H}^{ \pm}$corresponds to an interaction $\quad \mathcal{L}_{\mathrm{H}^{+} \mathrm{H}^{-} \mathrm{h}}=-g_{\mathrm{H}^{ \pm}} \frac{2 m_{\mathrm{H}^{ \pm}}^{2}}{v} \mathrm{H}^{+} \mathrm{H}^{-} \mathrm{h} . \quad g_{\mathrm{H}^{ \pm}} \quad$ depends on the details of the scalar potential that we do not address since this contribution can be safely neglected for $m_{\mathrm{H}^{ \pm}}>v$. 
TABLE I. Values of $A_{F}$ and $\hat{A}_{F}$ for charged fermions of the second and third generations; running masses at $\mu=m_{\mathrm{h}}[98]$ are used.

\begin{tabular}{lccc}
\hline \hline$f$ & $t$ & $b$ & $\tau$ \\
\hline$A_{F}\left(x_{f}\right)$ & 1.3796 & $-(4.37+4.75 i) 10^{-2}$ & $-(2.30+2.09 i) 10^{-2}$ \\
$\hat{A}_{F}\left(x_{f}\right)$ & 2.1010 & $-(4.78+4.76 i) 10^{-2}$ & $-(2.46+2.09 i) 10^{-2}$ \\
$f$ & $c$ & $s$ & $\mu$ \\
\hline$A_{F}\left(x_{f}\right)$ & $-(4.87+3.29 i) 10^{-3}$ & $-(8.99+3.89 i) 10^{-5}$ & $-(2.53+1.20 i) 10^{-4}$ \\
$\hat{A}_{F}\left(x_{f}\right)$ & $-(5.07+3.29 i) 10^{-3}$ & $-(9.15+3.89 i) 10^{-5}$ & $-(2.59+1.20 i) 10^{-4}$ \\
\hline \hline
\end{tabular}

TABLE II. Reference SM Higgs decay branching ratios (BR) for $m_{\mathrm{h}}=125 \mathrm{GeV}$; the total width is $\Gamma(\mathrm{h})=4.1 \mathrm{MeV}$.

\begin{tabular}{lccccc}
\hline \hline Channels $\bar{f} f$ & $\bar{b} b$ & $\bar{\tau} \tau$ & $\bar{c} c$ & $\bar{\mu} \mu$ & $\bar{s} s$ \\
\hline BR & 0.577 & $6.32 \times 10^{-2}$ & $2.91 \times 10^{-2}$ & $2.19 \times 10^{-4}$ & $2.46 \times 10^{-4}$ \\
Channels $V V$ & $\mathrm{gg}$ & $W W^{(*)}$ & $Z Z^{(*)}$ & $\gamma \gamma$ & $\gamma Z$ \\
\hline BR & $8.57 \times 10^{-2}$ & 0.215 & $2.64 \times 10^{-2}$ & $2.28 \times 10^{-3}$ & $1.54 \times 10^{-3}$ \\
\hline \hline
\end{tabular}

The decay into gluons $\mathrm{h} \rightarrow g g$ proceeds through similar diagrams, with the ones mediated by leptons and by $W$ and $\mathrm{H}^{ \pm}$bosons absent:

$$
\begin{aligned}
\Gamma(\mathrm{h} \rightarrow g g)_{\mathrm{gFC}-2 \mathrm{HDM}}= & \frac{\alpha_{S}^{2}}{128 \pi^{3}} \frac{m_{\mathrm{h}}^{3}}{v^{2}}\left(\left|\sum_{f} \frac{a_{f} v}{m_{f}} A_{F}\left(x_{f}\right)\right|^{2}\right. \\
& \left.+\left|\sum_{f} \frac{b_{f} v}{m_{f}} \hat{A}_{F}\left(x_{f}\right)\right|^{2}\right) .
\end{aligned}
$$

The couplings $a_{f}$ and $b_{f}$ in Eqs. (86) and (87) appear divided by fermion mass $m_{f}$ since the $A_{F}$ and $\hat{A}_{F}$ functions are defined including the mass factor of the $\mathrm{SM} \mathrm{h} \bar{f} f$ vertex. The loop functions are [92]

$$
\begin{array}{ll}
A_{F}(x)=-2 x[1+(1-x) f(x)], & \hat{A}_{F}(x)=-2 x f(x), \\
A_{V}(x)=2+3 x+3 x(2-x) f(x), & A_{S}(x)=x(1-x f(x)),
\end{array}
$$

where

$$
f(x)= \begin{cases}\arcsin ^{2}(1 / \sqrt{x}), & x \geq 1 \\ -\frac{1}{4}\left[\ln \left(\frac{1+\sqrt{1-x}}{1-\sqrt{1-x}}\right)-i \pi\right]^{2}, & x<1\end{cases}
$$

The dominant contribution in $\mathrm{h} \rightarrow \gamma \gamma$ comes from $A_{V}\left(x_{W}\right)=-8.339$. Other representative values of the functions are shown in Table I. It is important to stress that, while QCD corrections to Eq. (86) are small, that is not the case for Eq. (87) (see, e.g., Ref. [93]); we account for them by using

$$
\begin{aligned}
\Gamma(\mathrm{h} \rightarrow g g)_{\mathrm{gFC}-2 \mathrm{HDM}} \rightarrow & \frac{\Gamma(\mathrm{h} \rightarrow g g)_{\mathrm{gFC}-2 \mathrm{HDM}}}{\Gamma(\mathrm{h} \rightarrow g g)_{\mathrm{SM}}} \\
& \times \Gamma(\mathrm{h} \rightarrow g g)_{\mathrm{SM} \mathrm{ref}}
\end{aligned}
$$

with $\Gamma(\mathrm{h} \rightarrow g g)_{\mathrm{SM} \mathrm{ref}}=0.351 \mathrm{MeV}$ the $\mathrm{SM}$ reference value from Table II and $\frac{\Gamma(\mathrm{h} \rightarrow g g)_{g \mathrm{FC}-2 \mathrm{HDM}}}{\Gamma(\mathrm{h} \rightarrow g g)_{\mathrm{SM}}}$ computed according to Eq. (87) (for the SM denominator $\frac{a_{f} v}{m_{f}}=1, b_{f}=0$ ). For completeness, reference values of the SM Higgs decays [94-97] are reproduced in Table II.

\section{Production of $\boldsymbol{h}$}

In addition to the decay widths, production mechanisms are also modified. Besides VBF and associated production, already commented on [Eq. (80)], the most relevant one is gluon-gluon fusion ( $\mathrm{ggF}$ ) $g g \rightarrow \mathrm{h}$ [99]. The elementary process is the reverse of the decay $\mathrm{h} \rightarrow g g$, which is then convoluted with the gluon distribution functions in the proton (in the narrow-width approximation, production and decay are related straightforwardly). As in the case of the decay, Eq. (90), we incorporate QCD corrections by normalizing the SM prediction to the reference value in Table III, which shows reference cross sections for different production mechanisms [94-97].

TABLE III. Reference SM production cross sections for $m_{\mathrm{h}}=$ $125 \mathrm{GeV}$ (in pb).

\begin{tabular}{lcccccc}
\hline \hline & ggF & VBF & WH & ZH & ttH & bbH \\
\hline $8 \mathrm{TeV}$ & 19.27 & 1.578 & 0.7046 & 0.4153 & 0.1293 & 0.2035 \\
$13 \mathrm{TeV}$ & 43.92 & 3.748 & 1.380 & 0.8696 & 0.5085 & 0.5116 \\
$14 \mathrm{TeV}$ & 49.47 & 4.233 & 1.522 & 0.9690 & 0.6113 & 0.5805 \\
\hline \hline
\end{tabular}




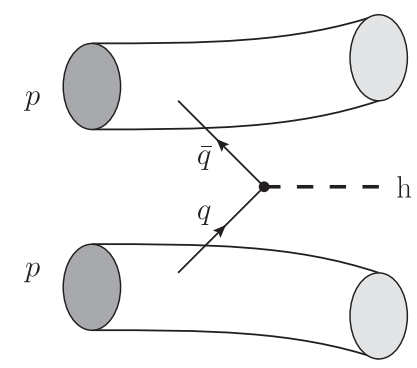

FIG. 1. $\quad \bar{q} q \rightarrow \mathrm{h}$ process.

We now turn to the direct $\bar{q} q \rightarrow \mathrm{h}$ production mechanism shown in Fig. 1. The motivation to consider this production mechanism is that, when $\left|n_{q}\right| \gg m_{q}$, the corresponding cross section may become inappropriately large; one is considering light quarks $q \neq t, b$. Sensitivity to enhanced Yukawa couplings of light quarks at the LHC has also been discussed, e.g., in Refs. [100-103].

For a generic Yukawa interaction $\mathcal{L}_{\mathrm{h} q q}=-\mathrm{h} \bar{q}\left(a_{q}+\right.$ $\left.i b_{q} \gamma_{5}\right) q$, the tree-level cross section for direct production $p p(\bar{q} q) \rightarrow \mathrm{h}$ is, in the narrow-width approximation,

$$
\sigma[p p(\bar{q} q) \rightarrow \mathrm{h}]=\left(\left|a_{q}\right|^{2}+\left|b_{q}\right|^{2}\right) \sigma_{0}(E) \mathcal{L}_{\bar{q} q}(E),
$$

where

$$
\begin{gathered}
\sigma_{0}(E) \equiv 2 \frac{\pi}{8 N_{c} E^{2}}=\left(\frac{\mathrm{TeV}}{E}\right)^{2} 101.8 \mathrm{pb}, \\
\mathcal{L}_{\bar{q} q}(E) \equiv \int_{x_{0}}^{1} d x f_{\bar{q}}^{p}\left(x, Q^{2}\right) f_{q}^{p}\left(x_{0} / x, Q^{2}\right) \frac{1}{x},
\end{gathered}
$$

with $E$ the center-of-mass proton energy, $f_{y}^{p}$ the distribution function of parton $y$ in the proton, $x_{0}=\frac{m_{\mathrm{h}}^{2}}{4 E^{2}}$, and $Q$ the factorization scale.

In Table IV, we collect the values of $\sigma[p p(\bar{q} q) \rightarrow \mathrm{h}]$ and $\mathcal{L}_{\bar{q} q}$ computed [104] for different quarks ${ }^{4}$ and setting $\left|a_{q}\right|^{2}+\left|b_{q}\right|^{2}=1$ in Eq. (91); this value of the couplings is obviously too large since it effectively corresponds, with respect to the $\mathrm{SM}$, to the change $m_{j} / v \mapsto v / v$, but it allows for easy use. Consider, for illustration, that for the LHC at $8 \mathrm{TeV} \sigma[p p(\bar{u} u) \rightarrow \mathrm{h}] \sim 10 \mathrm{pb}$; one can readily obtain

$$
\sigma[p p(\bar{u} u) \rightarrow \mathrm{h}] \sim 10 \mathrm{pb} \Leftrightarrow\left|a_{q}\right|^{2}+\left|b_{q}\right|^{2} \sim 7.3 \times 10^{-5} .
$$

Although considering $\sigma[p p(\bar{u} u) \rightarrow \mathrm{h}] \sim 10 \mathrm{pb}$ may be unrealistic (the total production cross section in Table III

\footnotetext{
${ }^{4}$ Equation (91) is obtained using the tree-level partonic cross section; furthermore, the results in Table IV are obtained by multiplying these simple predictions by a common $\mathcal{O}(1)$ factor (one for each LHC energy case), chosen such that $\sigma[p p(\bar{b} b) \rightarrow \mathrm{h}]$ in Eq. (91) reproduces the improved reference values in

\begin{tabular}{|c|c|c|c|c|c|c|c|c|}
\hline & \multicolumn{2}{|c|}{$\bar{d} d$} & \multicolumn{2}{|c|}{$\bar{u} u$} & \multicolumn{2}{|c|}{$\bar{s} s$} & \multicolumn{2}{|c|}{$\bar{c} c$} \\
\hline & $\mathcal{L}_{\bar{d} d}$ & $\sigma$ & $\mathcal{L}_{\bar{u} u}$ & $\sigma$ & $\mathcal{L}_{\bar{s} s}$ & $\sigma$ & $\mathcal{L}_{\bar{c} c}$ & $\sigma$ \\
\hline $8 \mathrm{TeV}$ & 14.56 & 16.60 & 21.53 & 24.53 & 4.41 & 5.02 & 2.65 & 3.01 \\
\hline $13 \mathrm{TeV}$ & 74.57 & 29.17 & 105.28 & 41.18 & 27.70 & 10.84 & 17.92 & 7.01 \\
\hline $14 \mathrm{TeV}$ & 95.49 & 31.53 & 133.90 & 44.21 & 36.46 & 12.04 & 23.83 & 7.87 \\
\hline
\end{tabular}
Refs. [94-97]. We also take $Q=E / 2$.
}

TABLE IV. $\quad \sigma[p p(\bar{q} q) \rightarrow \mathrm{h}]\left(\times 10^{3}\right)$ in $\mathrm{pb}$ and $\mathcal{L}_{\bar{q} q}\left(E^{2}\right)\left(\times 10^{3}\right)$ for different $\bar{q} q$.

for $8 \mathrm{TeV}$ is $\sim 22 \mathrm{pb}$ ), from Eq. (93), $\Gamma(\mathrm{h} \rightarrow u \bar{u}) \sim 1 \mathrm{MeV}$; even if it is a significant contribution to the width $\Gamma(\mathrm{h})$, it might still be compatible with the overall pattern of Higgs signal strengths. To our knowledge, there are no dedicated analyses of $\bar{q} q \rightarrow \mathrm{h}(q \neq b, t)$ from which experimental input can be used in this manner. However, it is reasonable to expect that this kind of production potentially "contaminates" the analyses of gluon-gluon fusion; in that case, one should add all $\sigma[p p(\bar{q} q) \rightarrow \mathrm{h}]$ contributions for light $q$ to the gluon-gluon fusion cross section when analyzing Higgs signal strengths. It is then clear that bounds more stringent than Eq. (93) would follow for the sum over all the different channels involved. The simple connection among the decays $\mathrm{h} \rightarrow \bar{q} q$ and the $\bar{q} q \rightarrow \mathrm{h}$ production mechanism-in the narrow-width approximation-that follows from Eqs. (85) and (91) is

$$
\frac{\sigma[p p(\bar{q} q) \rightarrow \mathrm{h}] / 1 \mathrm{pb}}{\Gamma(\mathrm{h} \rightarrow \bar{q} q) / 1 \mathrm{MeV}}=6.825\left(\frac{\mathrm{TeV}}{E}\right)^{2}\left(\frac{\mathcal{L}_{\bar{q} q}(E)}{10^{3}}\right),
$$

which allows for easy comparison of the relative strengths of the constraints imposed by $\bar{q} q \rightarrow \mathrm{h}$ production and $\mathrm{h} \rightarrow \bar{q} q$ decay for light quarks $q$.

\section{Constraints}

The main source of experimental constraints that we use is the combined analysis of LHC-Run I data from the ATLAS and CMS collaborations in Ref. [91], which provides detailed information on (production) $\times$ (decay) of the $125 \mathrm{GeV}$ Higgs $\mathrm{h}$ for:

(i) production: $g g F, V B F$, associated $W \mathrm{~h}, Z \mathrm{~h}$, and $t \mathrm{th}$;

(ii) decay: $\mathrm{h} \rightarrow \gamma \gamma, Z Z, W W, \tau \bar{\tau}$ and $b \bar{b}$.

Results from LHC-Run II in specific channels like (associated $V \mathrm{~h}) \times(\mathrm{h} \rightarrow b \bar{b})[105,106]$ or $(\mathrm{ggF}+\mathrm{VBF}) \times(\mathrm{h} \rightarrow \tau \bar{\tau})$ [107] are starting to improve over Ref. [91]. One should also consider off-shell $(\mathrm{ggF}+\mathrm{VBF}) \rightarrow \mathrm{h}^{(*)} \rightarrow W W^{(*)}$ constraints on the total width $\Gamma(\mathrm{h})$ [108], even if they are still weak $[109,110]$. Finally, dedicated studies like Refs. [111,112] put useful bounds on $\mathrm{h} \rightarrow \mu^{+} \mu^{-}, e^{+} e^{-}$.

\section{Electric dipole moments}

As discussed at the end of Sec. II, nonreal $\mathcal{N}_{\mathrm{f}}$ matrices are a source of $C P$ violation in scalar-fermion interactions, which can induce electric dipole moments (EDMs). Consider, 


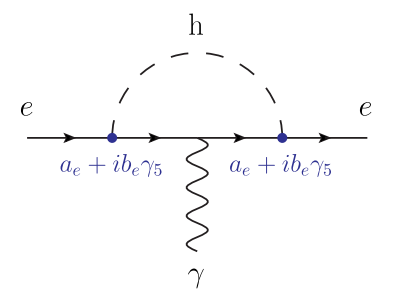

FIG. 2. h-mediated contribution to $d_{e}$ at one loop.

e.g., an electron-Higgs coupling $\mathcal{L}_{\text {hee }}=-\mathrm{h} \bar{e}\left(a_{e}+i b_{e} \gamma_{5}\right) e$; the one-loop diagram in Fig. 2 gives a contribution to the electron $\mathrm{EDM} d_{e}$ :

$$
d_{e}=\frac{3 m_{e} a_{e} b_{e}}{16 \pi^{2} m_{\mathrm{h}}^{2}}\left(1+\mathcal{O}\left(\frac{m_{e}^{2}}{m_{\mathrm{h}}^{2}}\right)\right) .
$$

It is to be noticed that, for $a_{e} \sim b_{e} \sim m_{e} / v$, Eq. (95) gives $d_{e} \sim 10^{-34} \mathrm{e} \cdot \mathrm{cm}$. When $\left|a_{e}\right|,\left|b_{e}\right| \gg m_{e} / v$ are a priori allowed, up to the effect of other constraints, a significant enhancement in $d_{e}$ can be expected. For current experimental bounds $\left|d_{e}\right|<10^{-27} \mathrm{e} \cdot \mathrm{cm}$, considering only this contribution gives

$$
a_{e} b_{e}<8 \times 10^{-5},
$$

or, with Eqs. (81) and (82) and neglecting $m_{e}$ with respect to $c_{\beta \alpha} n_{e}$,

$$
c_{\beta \alpha}^{2} \operatorname{Re}\left(n_{e}\right) \operatorname{Im}\left(n_{e}\right)<5 \mathrm{GeV}^{2} .
$$

Anticipating results from Sec. IV D, in particular Fig. 4(g), it is clear that the bounds imposed by the LHC results are more stringent than Eq. (97). It should also be noticed that including contributions analogous to Fig. 2 with $\mathrm{h} \rightarrow \mathrm{H}$, A, gives

$$
\operatorname{Re}\left(n_{e}\right) \operatorname{Im}\left(n_{e}\right)\left(c_{\beta \alpha}^{2}+s_{\beta \alpha}^{2} \frac{m_{\mathrm{h}}^{2}}{m_{\mathrm{H}}^{2}}+\frac{m_{\mathrm{h}}^{2}}{m_{\mathrm{A}}^{2}}\right)<5 \mathrm{GeV}^{2}
$$

and does not change this conclusion. Furthermore, one-loop contributions with virtual $\mathrm{H}^{ \pm}$and neutrinos are suppressed.

It is well known that two-loop "Barr-Zee" [113-118] contributions can be significant; studies such as Refs. [119,120] address such constraints on $C P$-violating Higgs-fermion couplings. However, those contributions involve different $n_{f}$ couplings simultaneously, together with the masses of the different scalars, preventing a simple translation into bounds on a single parameter. It is to be noticed, too, that cancellations among different diagrams in that class may occur $[67,121]$. Including such a kind of analysis is beyond the scope of this work; in any case, one should keep in mind that the analysis of EDMs may have some impact on the results of Sec. IV D. The previous discussion also applies to the EDMs of the $u$ and $d$ quarks and the experimental constraints that the neutron EDM bounds impose, including, in addition, the impact of QCD effects [122].

\section{Analysis}

With the deviations with respect to the SM of the couplings of $\mathrm{h}$ and their implications for decays and production mechanisms, one can impose the experimental constraints of Sec. IV B 3 and explore the allowed values of $c_{\beta \alpha}$ and the gFC parameters $n_{f}$ in Eq. (38). For the results presented in the following, we consider the most conservative situation; i.e., all parameters are free to vary simultaneously. Compared to restricted situations in which not all parameters are considered simultaneously, this offers a safer interpretation of excluded regions (they are excluded whatever the values of the parameters not displayed) at the price, of course, of larger allowed regions.

Figure 3 shows $n_{f}$ vs $c_{\beta \alpha}$ for all quarks and leptons. Some comments are in order:

(i) As expected, for $c_{\beta \alpha} \rightarrow 0$, the constraints on $n_{f}$ disappear.

(ii) For $u, c, d$, and $s$ quarks, the allowed regions are almost identical, as one could anticipate from their irrelevant role, within the SM, in the available production $\times$ decay Higgs signal strengths. The corresponding $n_{f}$ 's appear to be effectively limited by the contributions to the Higgs width.

(iii) Surprisingly, the allowed size of $\left|n_{t}\right|$ appears to be independent of $c_{\beta \alpha}$; this will be discussed in connection with Fig. 4(c) below.

(iv) The $n_{b}$ and $n_{\tau}$ cases are also similar, with allowed regions differing from the $u, c, d$, and $s$ cases for $\left|n_{q}\right|$ 's below $10-15 \mathrm{GeV}$ and not small $c_{\beta \alpha}$.

(v) For $n_{e}$ and $n_{\mu}$, the allowed regions are much more constrained, owing to the bounds set by dedicated $p p \rightarrow \mathrm{h} \rightarrow e^{+} e^{-}, \mu^{+} \mu^{-}$analyses such as Refs. [111,112].

Although Fig. 3 shows absolute bounds on $\left|n_{f}\right|$ 's, it does not give information on $\arg \left(n_{f}\right)$ 's and cannot be directly read in terms of the scalar and pseudoscalar couplings of $h$ in Eqs. (81) and (82). Considering that, Fig. 4 shows $\bar{b}_{f}$ vs $\bar{a}_{f}$ with

$$
\bar{a}_{f} \equiv s_{\beta \alpha} m_{f}+c_{\beta \alpha} \operatorname{Re}\left(n_{f}\right), \quad \bar{b}_{f}=c_{\beta \alpha} \operatorname{Im}\left(n_{f}\right) .
$$

Furthermore, to maintain some information on $c_{\beta \alpha}$, allowed regions corresponding to $\left|c_{\beta \alpha}\right|<0.01$, to $0.01<\left|c_{\beta \alpha}\right|<0.1$, and to $0.1<\left|c_{\beta \alpha}\right|$ are displayed. One can notice that:

(i) for the first and second fermion generations, there is no dependence on $\arg \left(n_{f}\right)$, since only decays, with rates proportional to $\left|\bar{a}_{f}\right|^{2}+\left|\bar{b}_{f}\right|^{2}$, are relevant. For quarks, the allowed region for $\left|c_{\beta \alpha}\right|<0.01$ is smaller; this is simply due to the perturbativity requirement in Eq. (79). 


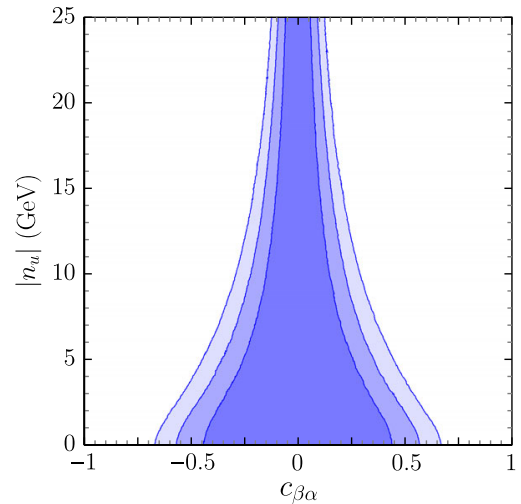

(a) $u$

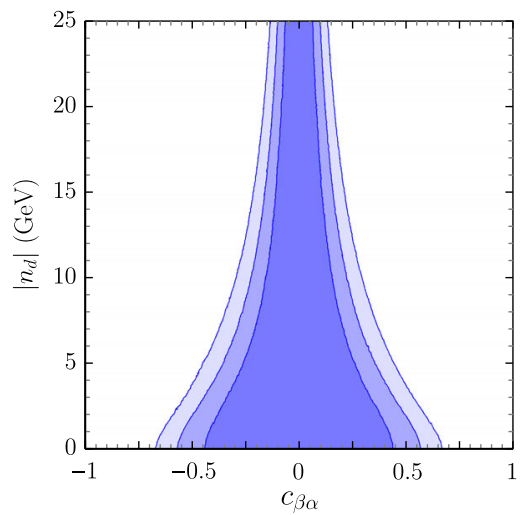

(d) $d$

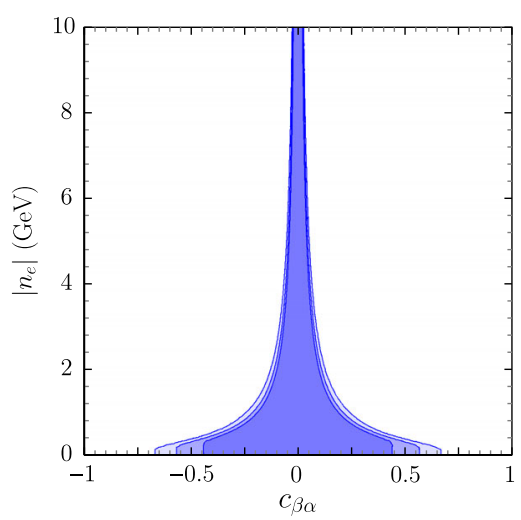

(g) $e$

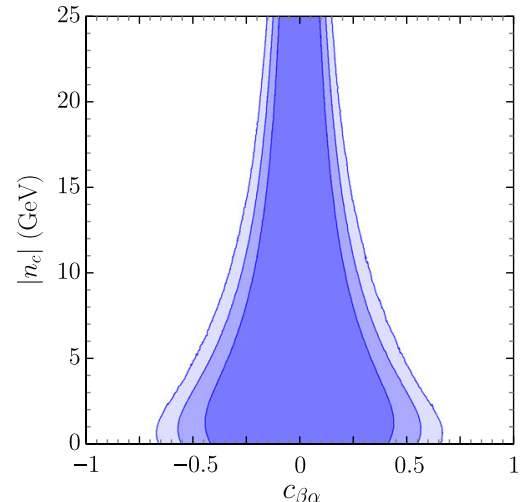

(b) $c$

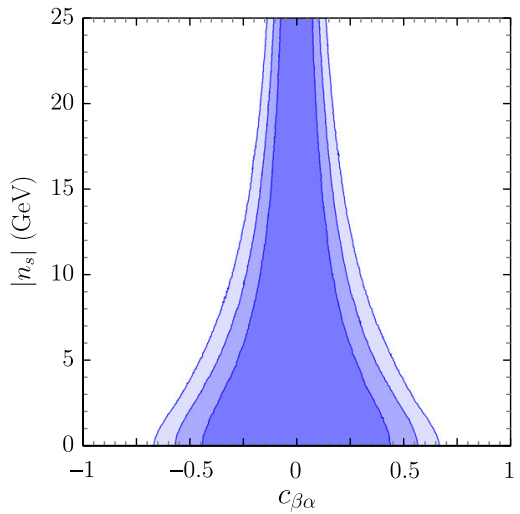

(e) $s$

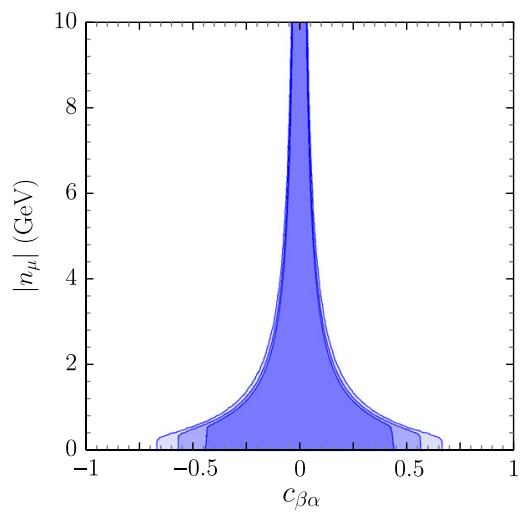

(h) $\mu$

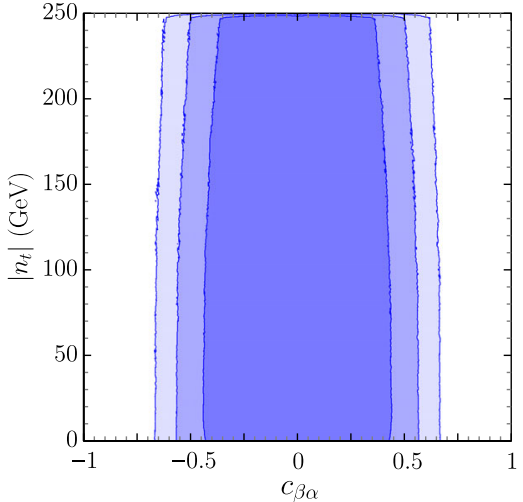

(c) $t$

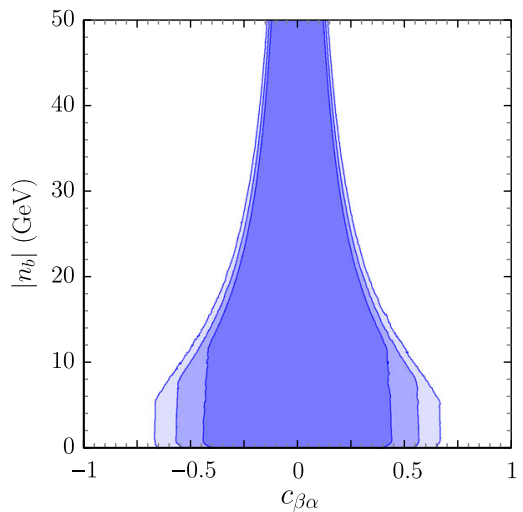

(f) $b$

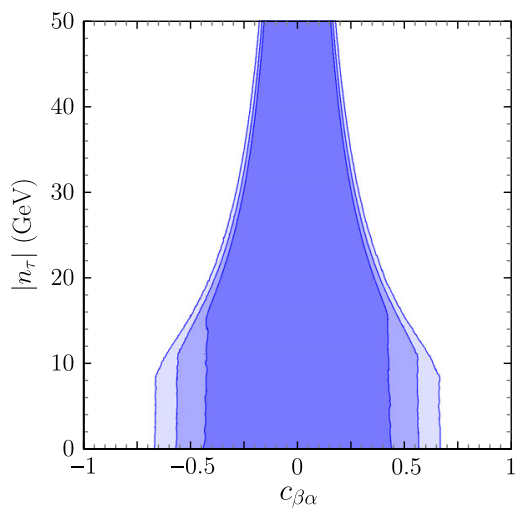

(i) $\tau$

FIG. 3. $\left|n_{f}\right|$ vs $c_{\beta \alpha}$ for the different fermions $f$; darker to lighter regions correspond to $68 \%$, 95\%, and $99 \%$ C.L.

(ii) For the top quark, two separate regions are allowed; this is also expected since independent sign changes in both $\bar{a}_{t}$ and $\bar{b}_{t}$ (together with sign changes in $c_{\beta \alpha}$, $s_{\beta \alpha}$ ) do not alter the predictions. For $\left|c_{\beta \alpha}\right|<0.01$, the allowed regions are quite reduced and placed around $\left(\bar{a}_{t}, \bar{b}_{t}\right)=\left( \pm m_{t}, 0\right)$; with $0.01<\left|c_{\beta \alpha}\right|<0.1$, their size increases, and only for $\left|c_{\beta \alpha}\right|>0.1$, the interplay of (i) pseudoscalar contributions to $g g \rightarrow \mathrm{h}$ and $\mathrm{h} \rightarrow \gamma \gamma$ and (ii) $W$-top(scalar) interference in $\mathrm{h} \rightarrow \gamma \gamma$ gives rise to larger regions. (iii) For $b$ and $\tau$, the regions for not-too-small mixing, $\left|c_{\beta \alpha}\right|>0.01$, are ring shaped; $m_{b}$ and $m_{\tau}$ set the radii of such regions, as could be expected from the agreement of $\mathrm{h} \rightarrow b \bar{b}$ and $\mathrm{h} \rightarrow \tau \bar{\tau}$ signal strengths with SM expectations. For small mixing, $\left|c_{\beta \alpha}\right|<0.01$, the perturbativity requirement on $\left|n_{b}\right|$ and $\left|n_{\tau}\right|$ limits the allowed departure from $\left(\bar{a}_{f}, \bar{b}_{f}\right)=\left( \pm m_{f}, 0\right)$, giving in fact, for the $b$ case, two disjoint patches. To close this section, we recall the discussion on $\bar{q} q \rightarrow \mathrm{h}$ production in Sec. IV B 2; as commented there, values of 


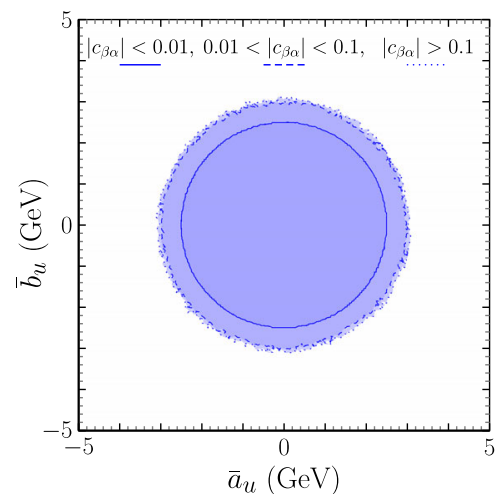

(a) $u$

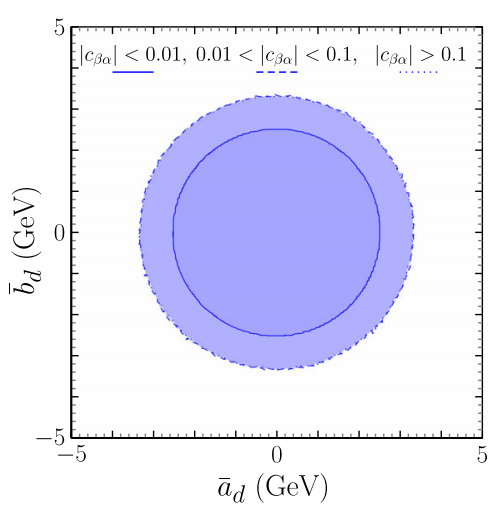

(d) $d$

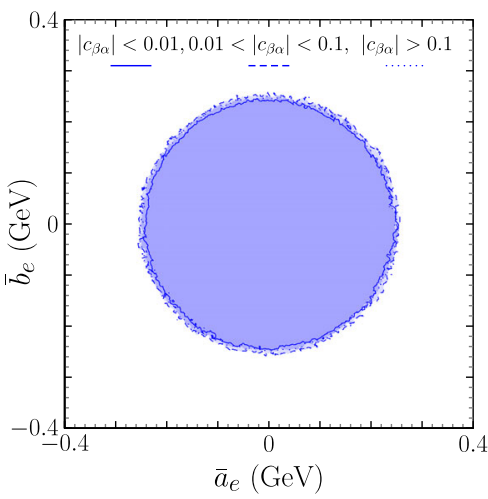

(g) $e$

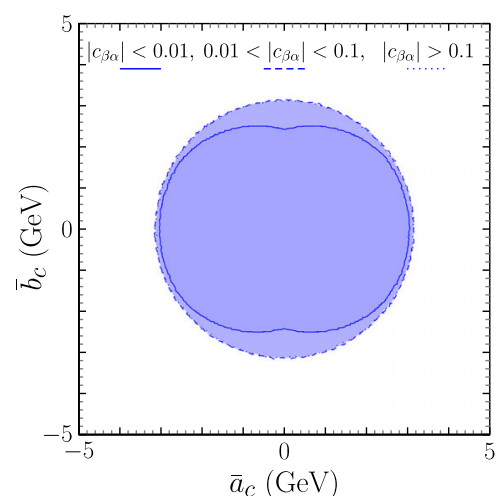

(b) $c$

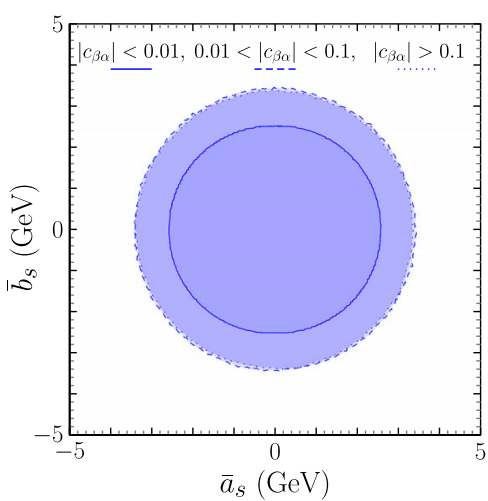

(e) $s$

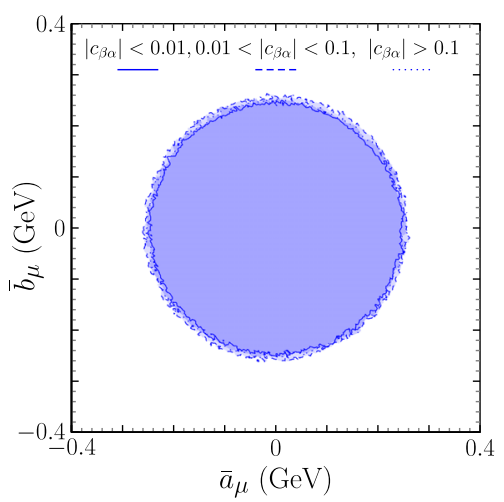

(h) $\mu$

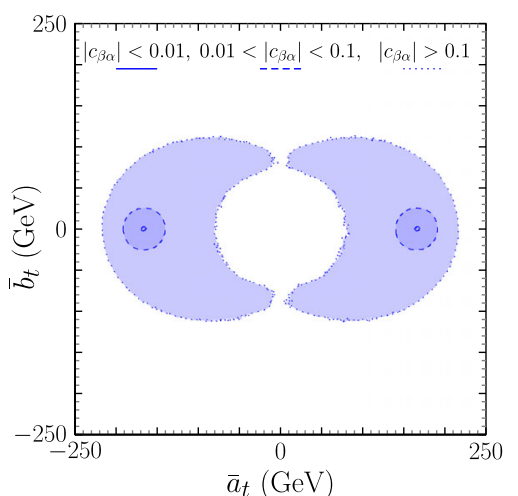

(c) $t$

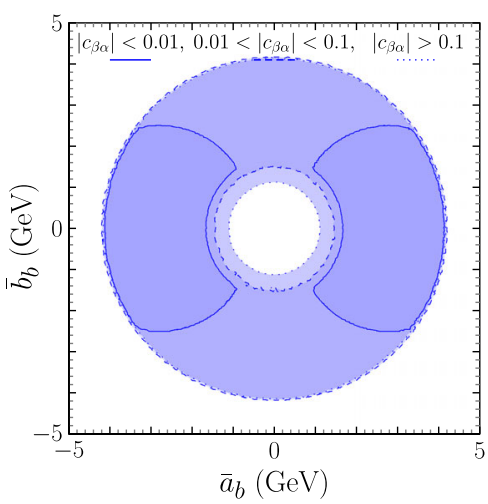

(f) $b$

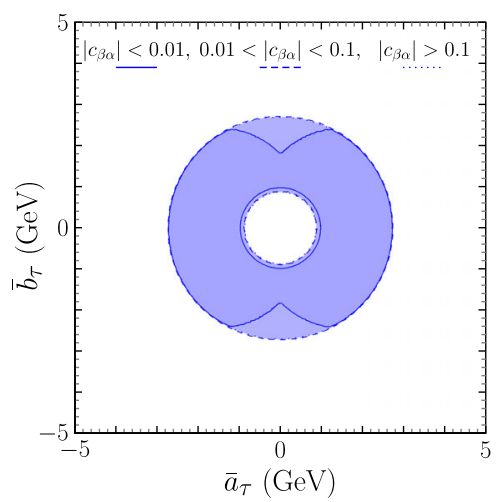

(i) $\tau$

FIG. 4. Allowed regions at $99 \%$ C.L. for pseudoscalar vs scalar couplings for the different fermions $f$ with $\mathcal{L}_{\mathrm{h} f f}=-\frac{\mathrm{h}}{v} \bar{f}\left(\bar{a}_{f}+i \bar{b}_{f} \gamma_{5}\right) f$.

$n_{f}$ in agreement with the SM-like Higgs signal strengths could potentially give production cross sections not far from the dominating SM ones. Figure 5 shows

$$
\begin{aligned}
& \frac{\sigma[q \bar{q} \mathrm{~h}]}{\sigma[p p(g g) \rightarrow \mathrm{h}]_{\mathrm{SM}}} \\
& \equiv\left(\sum_{q=u, c, d, s} \sigma[p p(q \bar{q}) \rightarrow \mathrm{h}]\right) / \sigma[p p(g g) \rightarrow \mathrm{h}]_{\mathrm{SM}},
\end{aligned}
$$

vs the total Higgs width and vs the gluon-gluon fusion production cross section in two different analyses: in Figs. 5(a) and 5(b), $\sigma[q \bar{q} \mathrm{~h}]$ is added to the ggF production cross section, while in Figs. 5(c) and 5(d), it is not (and therefore, in the analysis, it does not directly affect observables constrained by experiment). Comparing Figs. 5(a) and 5(b) with 5(c) and 5(d), one can notice that the constraints from Higgs signal strengths are able to bound the size of $\sigma[q \bar{q} \mathrm{~h}]$, even if there is room for an overall $q \bar{q} \rightarrow \mathrm{h}$ cross section, which is quite sizable, not far 


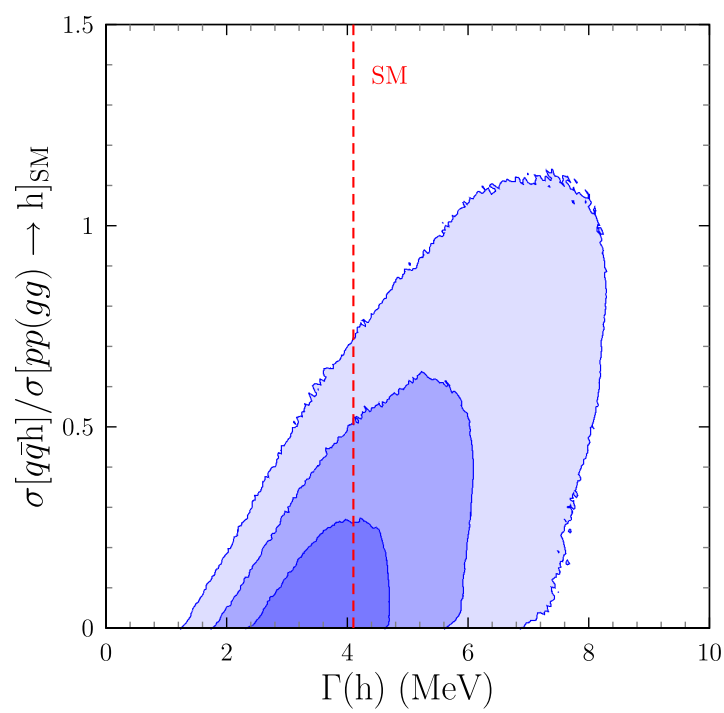

(a) $\sigma[q \bar{q} \mathrm{~h}]$ vs. $\Gamma(\mathrm{h}), \sigma[q \bar{q} \mathrm{~h}] \subset \mathrm{ggF}$.

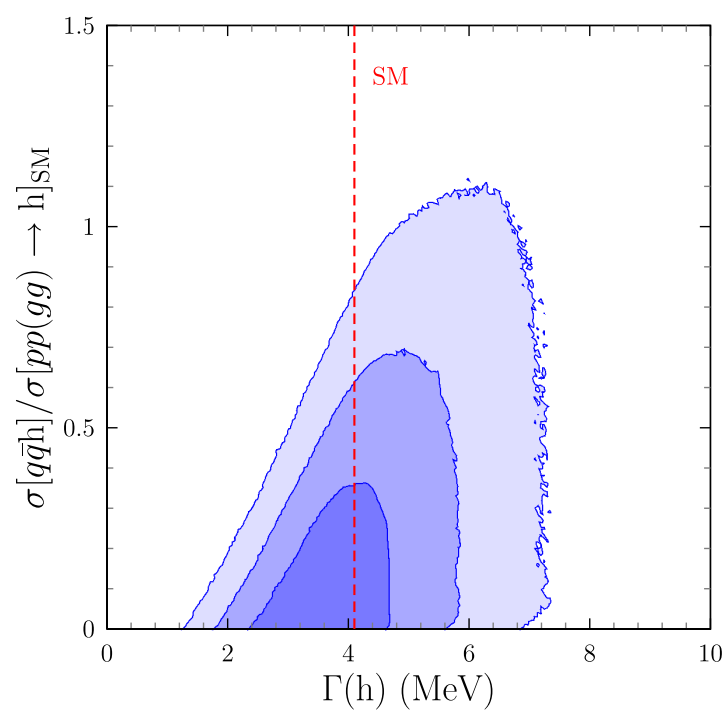

(c) $\sigma[q \bar{q} \mathrm{~h}]$ vs. $\Gamma(\mathrm{h}), \sigma[q \bar{q} \mathrm{~h}] \not \subset \mathrm{ggF}$

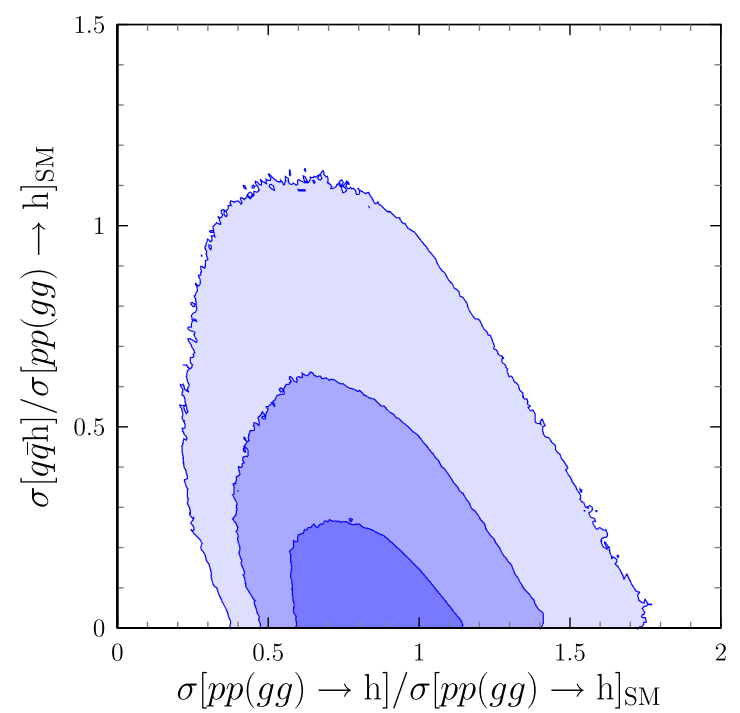

(b) $\sigma[q \bar{q} \mathrm{~h}]$ vs. $\sigma[p p(g g) \rightarrow \mathrm{h}], \sigma[q \bar{q} \mathrm{~h}] \subset \operatorname{ggF}$

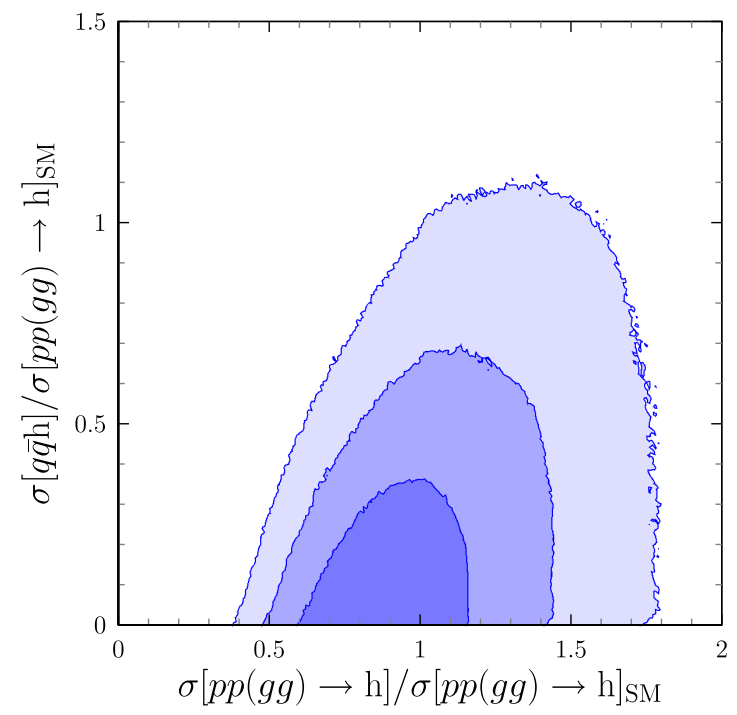

(d) $\sigma[q \bar{q} \mathrm{~h}]$ vs. $\sigma[p p(g g) \rightarrow \mathrm{h}], \sigma[q \bar{q} \mathrm{~h}] \not \subset \operatorname{ggF}$

FIG. 5. Effect of including $q \bar{q} \rightarrow \mathrm{h}$ production in ggF in analyses of Higgs signal strengths; darker to lighter regions correspond to $68 \%, 95 \%$, and $99 \%$ C.L.

from the complete SM Higgs production cross section. Furthermore, when $\sigma[q \bar{q} \mathrm{~h}]$ is added to the ggF production cross section, the agreement with the observed Higgs signal strengths allows for a smaller amount of $q \bar{q} \rightarrow \mathrm{h}$, and for sizable $q \bar{q} \rightarrow \mathrm{h}$, it is achieved at the cost of (i) reducing the $\mathrm{ggF}$ production cross section and (ii) increasing the total width $\Gamma(\mathrm{h})$, as the shape of the allowed regions in Figs. 5(a) and 5(b) shows. For the results in Figs. 3 and 4, the bounds on the different $\bar{a}_{f}$ and $\bar{b}_{f}$ do not differ in either analysis. It should be finally mentioned that, in connection with the previous comments and the size of $\sigma[p p(q \bar{q}) \rightarrow \mathrm{h}]$, it might be interesting to analyze, for the remaining neutral scalars $\mathrm{H}$ and $\mathrm{A}$, the cross sections for $p p(q \bar{q}) \rightarrow \mathrm{H}, \mathrm{A}$ at the LHC.

\section{E. Other flavor-conserving models}

In this section, we present, for comparison, results corresponding to other flavor-conserving 2HDMs: types I, II, X (or lepton specific), and Y (or flipped) in Sec. IV E 1 and the aligned 2HDMs in Sec. IV E2. The analyses are performed by imposing the same constraints used above in the analysis of the gFC scenario.

\section{1. $\mathbb{Z}_{2} 2 H D M s$}

In 2HDMs of types I, II, X, and Y, there is only one Yukawa coupling matrix in each secctor (up and down quarks and charged leptons), and consequently, the $\mathcal{N}_{\mathrm{f}}$ 


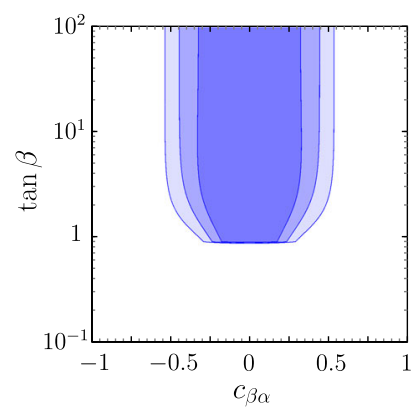

(a)2HDM I.

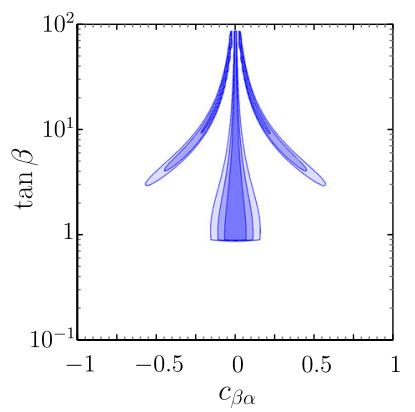

(b)2HDM II.

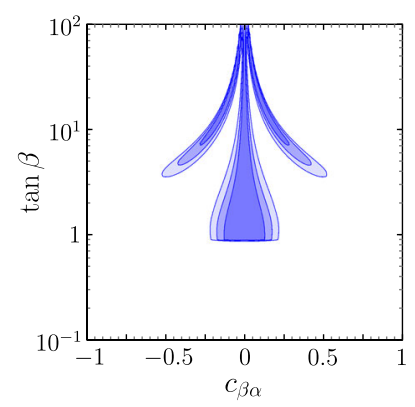

(c)2HDM X.

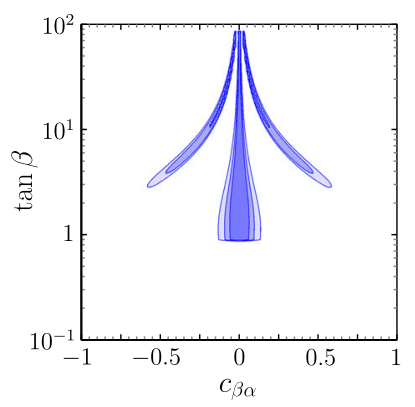

(d) $2 \mathrm{HDM}$ Y.

FIG. 6. $t_{\beta}$ vs $c_{\beta \alpha}$; darker to lighter regions correspond to $68 \%, 95 \%$, and $99 \%$ C.L.

matrices are proportional to the corresponding mass matrices $\mathcal{M}_{\mathrm{f}}$, as shown in Eqs. (101)-(104):

2HDM I: $\mathcal{N}_{\mathrm{u}}=t_{\beta}^{-1} \mathcal{M}_{\mathrm{u}}, \quad \mathcal{N}_{\mathrm{d}}=t_{\beta}^{-1} \mathcal{M}_{\mathrm{d}}, \quad \mathcal{N}_{\ell}=t_{\beta}^{-1} \mathcal{M}_{\ell}$,

2HDM II $: \mathcal{N}_{\mathrm{u}}=t_{\beta}^{-1} \mathcal{M}_{\mathrm{u}}, \quad \mathcal{N}_{\mathrm{d}}=-t_{\beta} \mathcal{M}_{\mathrm{d}}, \quad \mathcal{N}_{\ell}=-t_{\beta} \mathcal{M}_{\ell}$,

$2 \operatorname{HDM~X:} \mathcal{N}_{\mathrm{u}}=t_{\beta}^{-1} \mathcal{M}_{\mathrm{u}}, \quad \mathcal{N}_{\mathrm{d}}=t_{\beta}^{-1} \mathcal{M}_{\mathrm{d}}, \quad \mathcal{N}_{\ell}=-t_{\beta} \mathcal{M}_{\ell}$,

$2 \mathrm{HDM} \mathrm{Y}: \mathcal{N}_{\mathrm{u}}=t_{\beta}^{-1} \mathcal{M}_{\mathrm{u}}, \quad \mathcal{N}_{\mathrm{d}}=-t_{\beta} \mathcal{M}_{\mathrm{d}}, \quad \mathcal{N}_{\ell}=t_{\beta}^{-1} \mathcal{M}_{\ell}$.
The only parameters relevant for the analyses are $c_{\beta \alpha}$ and $t_{\beta}$. The allowed regions for $t_{\beta}$ vs $c_{\beta \alpha}$ are shown in Fig. 6. It is to be noticed that $t_{\beta} \ll 1$ is not allowed because of the perturbativity constraint on the top-quark Yukawa couplings; with $\mathcal{M}_{\mathrm{u}}=\frac{v}{\sqrt{2}} s_{\beta} \Delta_{2}$, the constraint trivially gives $\frac{\sqrt{2} m_{t}}{v s_{\beta}}<\mathcal{O}(1)$. In model I, the there is no constraint on $t_{\beta} \gg 1$; in models II and $\mathrm{Y}$, the perturbativity constraint due to $m_{b}$ does limit $t_{\beta} \gg 1$, while, similarly, in model $\mathrm{X}$, it is $m_{\tau}$ that limits $t_{\beta} \gg 1$. For the different $\mathcal{L}_{\mathrm{h} f f}=-\frac{\bar{a}_{f}}{v} \mathrm{~h} \bar{f} f$ interactions, in models I, II, X, and Y, either $\frac{\bar{a}_{f}}{m_{f}}=$ $s_{\beta \alpha}+t_{\beta}^{-1} c_{\beta \alpha}$ or $\frac{\bar{a}_{f}}{m_{f}}=s_{\beta}-t_{\beta} c_{\beta \alpha}$, identical for all three generations in each sector:

2HDM I: $\quad \frac{\bar{a}_{u}}{m_{u}}=s_{\beta \alpha}+t_{\beta}^{-1} c_{\beta \alpha}, \quad \frac{\bar{a}_{d}}{m_{d}}=s_{\beta \alpha}+t_{\beta}^{-1} c_{\beta \alpha}, \quad \frac{\bar{a}_{\ell}}{m_{\ell}}=s_{\beta \alpha}+t_{\beta}^{-1} c_{\beta \alpha}$,

2HDM II: $\quad \frac{\bar{a}_{u}}{m_{u}}=s_{\beta \alpha}+t_{\beta}^{-1} c_{\beta \alpha}, \quad \frac{\bar{a}_{d}}{m_{d}}=s_{\beta}-t_{\beta} c_{\beta \alpha}, \quad \frac{\bar{a}_{\ell}}{m_{\ell}}=s_{\beta}-t_{\beta} c_{\beta \alpha}$,

2HDM X: $\frac{\bar{a}_{u}}{m_{u}}=s_{\beta \alpha}+t_{\beta}^{-1} c_{\beta \alpha}, \quad \frac{\bar{a}_{d}}{m_{d}}=s_{\beta \alpha}+t_{\beta}^{-1} c_{\beta \alpha}, \quad \frac{\bar{a}_{\ell}}{m_{\ell}}=s_{\beta \alpha}-t_{\beta} c_{\beta \alpha}$,

2HDM Y: $\frac{\bar{a}_{u}}{m_{u}}=s_{\beta \alpha}+t_{\beta}^{-1} c_{\beta \alpha}, \quad \frac{\bar{a}_{d}}{m_{d}}=s_{\beta \alpha}-t_{\beta} c_{\beta \alpha}, \quad \frac{\bar{a}_{\ell}}{m_{\ell}}=s_{\beta \alpha}+t_{\beta}^{-1} c_{\beta \alpha}$.

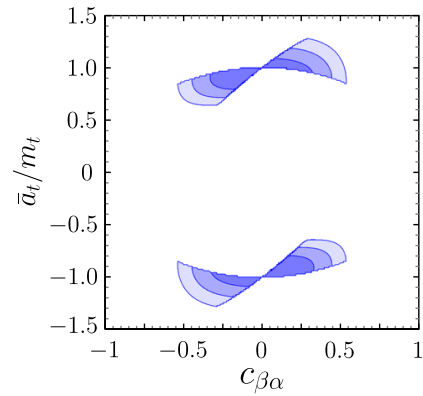

(a)2HDM I.

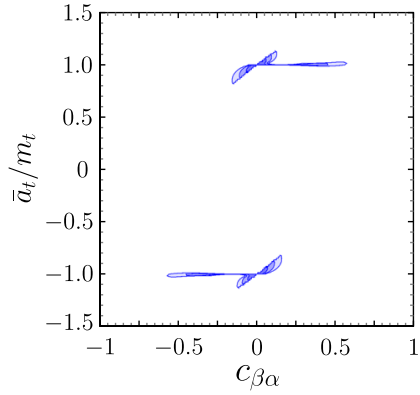

(b) $2 \mathrm{HDM}$ II.

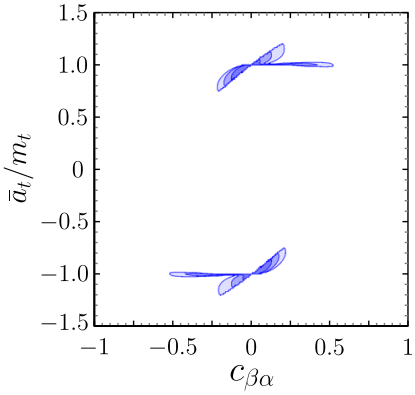

(c) $2 \mathrm{HDM} \mathrm{X}$.

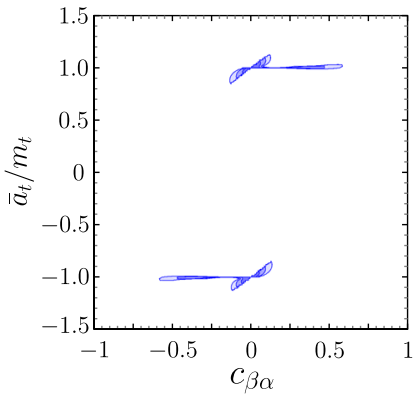

(d)2HDM Y.

FIG. 7. $\bar{a}_{u} / m_{u}$ vs $c_{\beta \alpha}$; darker to lighter regions correspond to $68 \%, 95 \%$, and $99 \%$ C.L. 


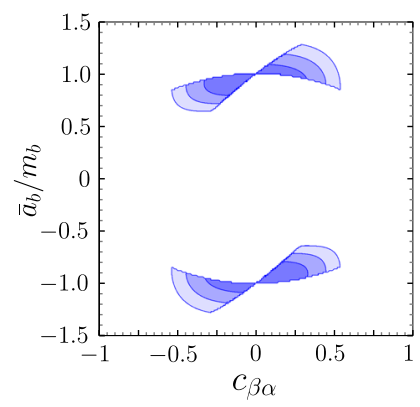

(a) 2 HDM I.

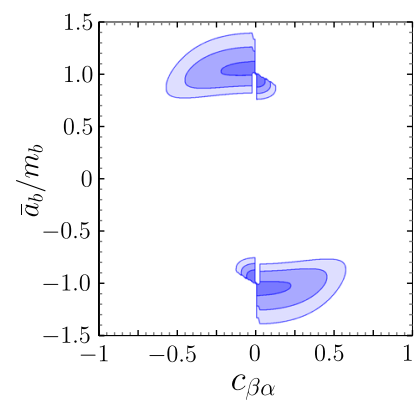

(b)2HDM II.

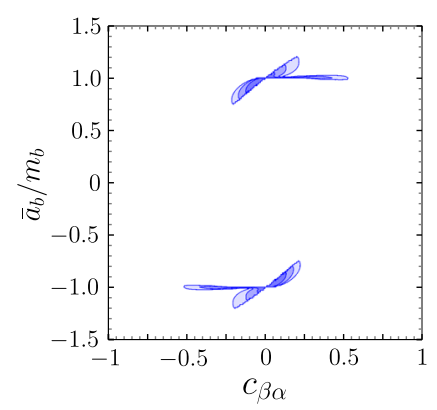

(c)2HDM X.

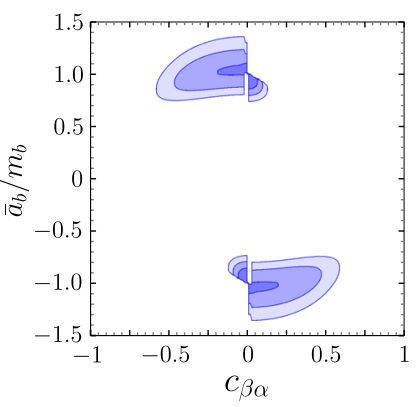

(d)2HDM Y.

FIG. 8. $\bar{a}_{d} / m_{d}$ vs $c_{\beta \alpha}$; darker to lighter regions correspond to $68 \%, 95 \%$, and $99 \%$ C.L.

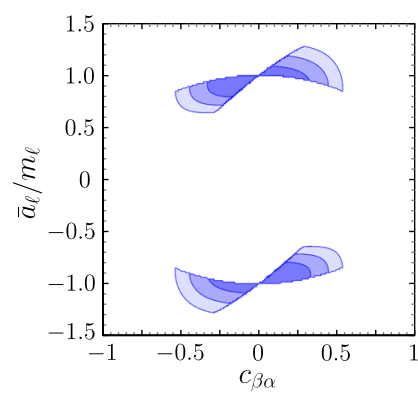

(a)2HDM I.

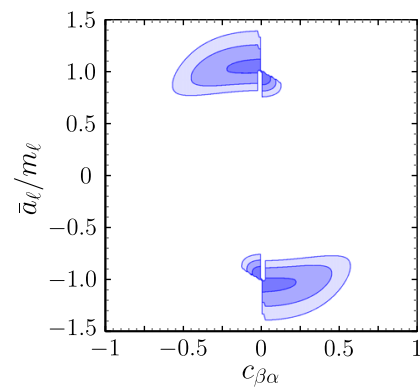

(b)2HDM II.

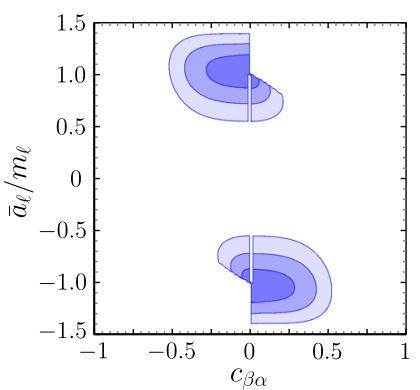

(c)2HDM X.

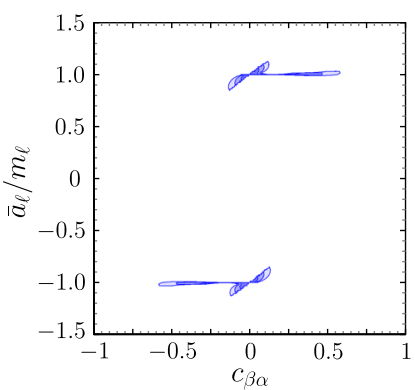

(d)2HDM Y.

FIG. 9. $\bar{a}_{\ell} / m_{\ell}$ vs $c_{\beta \alpha}$; darker to lighter regions correspond to $68 \%, 95 \%$, and $99 \%$ C.L.

There is no pseudoscalar interaction term. Figures 7-9 show $\bar{a}_{f} / m_{f}$ vs $c_{\beta \alpha}$ in each sector for the different models. It is clear that, overall, the allowed regions remain close to $\frac{\bar{a}_{f}}{m_{f}}= \pm 1$ for all $c_{\beta \alpha}$ and $t_{\beta}$ values, in clear contrast to Fig. 4 in the $\mathrm{gFC}$ scenario.

\section{Aligned 2HDM}

In the aligned 2HDM,

$$
\Delta_{2}=\xi_{u} \Delta_{1}, \quad \Gamma_{2}=\xi_{d} \Gamma_{1}, \quad \Pi_{2}=\xi_{\ell} \Pi_{1} .
$$

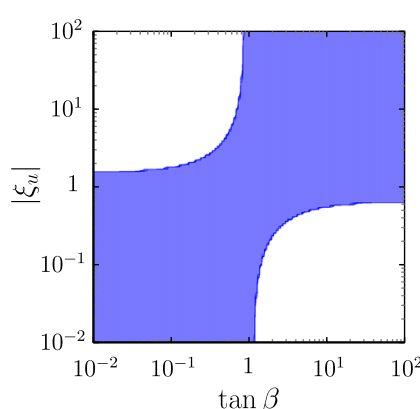

(a) $\left|\xi_{u}\right|$ vs $t_{\beta}$.

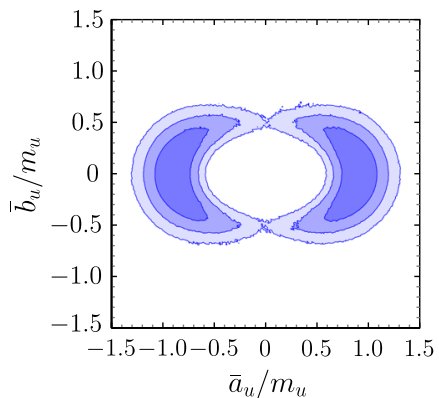

(b) $\bar{b}_{u} / m_{u}$ vs. $\bar{a}_{u} / m_{u}$.
The perturbativity constraint on the top-quark Yukawa couplings reads, with $\mathcal{M}_{\mathrm{u}}^{0}=\frac{v}{\sqrt{2}}\left(c_{\beta}+s_{\beta} \xi_{u}\right) \Delta_{1}=$ $\frac{v}{\sqrt{2}} \xi_{u}^{-1}\left(c_{\beta}+s_{\beta} \xi_{u}\right) \Delta_{2}$

$$
\frac{\sqrt{2} m_{t}}{v\left|c_{\beta}+s_{\beta} \xi_{u}\right|}, \frac{\sqrt{2} m_{t}\left|\xi_{u}\right|}{v\left|c_{\beta}+s_{\beta} \xi_{u}\right|}<\mathcal{O}(1)
$$

that is, roughly, $\left|c_{\beta}+s_{\beta} \xi_{u}\right|,\left|c_{\beta} \xi_{u}^{-1}+s_{\beta}\right|>1$, which imposes the shape of the allowed $\left|\xi_{u}\right|$ vs $t_{\beta}$ region in Fig. 10(a) (for $\xi_{d}$ and $\xi_{\ell}$, regions with similar shape also follow, with $m_{t} \rightarrow m_{b}, m_{\tau}$ rescalings). Contrary to models

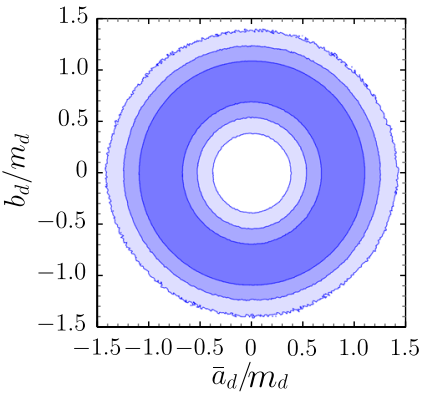

(c) $\bar{b}_{d} / m_{d}$ vs. $\bar{a}_{d} / m_{d}$.

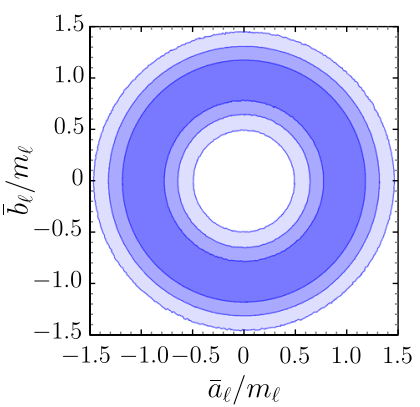

(d) $\bar{b}_{\ell} / m_{\ell}$ vs. $\bar{a}_{\ell} / m_{\ell}$.

FIG. 10. Aligned; darker to lighter regions correspond to $68 \%, 95 \%$, and $99 \%$ C.L. 
I, II, X, and Y, the aligned 2HDM allows for the simultaneous presence of scalar and pseudoscalar interactions, $\mathcal{L}_{\mathrm{h} f f}=-\frac{\mathrm{h}}{v} \bar{f}\left(\bar{a}_{f}+i \bar{b}_{f} \gamma_{5}\right) f$. However, as in models I, II, X, and $\mathrm{Y}$, in each sector, $\frac{\bar{a}_{f}}{m_{f}}$ and $\frac{\bar{b}_{f}}{m_{f}}$ are identical for all three generations:

$$
\begin{aligned}
\frac{\bar{a}_{f}}{m_{f}} & =s_{\beta \alpha}+c_{\beta \alpha} \frac{\left(1-t_{\beta}^{2}\right) \operatorname{Re}\left(\xi_{f}\right)+\left(\left|\xi_{f}\right|^{2}-1\right) t_{\beta}}{\left|1+t_{\beta} \xi_{f}\right|^{2}}, \\
\frac{\bar{b}_{f}}{m_{f}} & =c_{\beta \alpha}\left(1+t_{\beta}^{2}\right) \frac{\operatorname{Im}\left(\xi_{f}\right)}{\left|1+t_{\beta} \xi_{f}\right|^{2}} .
\end{aligned}
$$

Figures 10(b)-10(d) show $\frac{\bar{b}_{f}}{m_{f}}$ vs $\frac{\bar{a}_{f}}{m_{f}}$ allowed regions for each sector. It is to be noticed that the allowed regions are similar to the ones for third-generation fermions in Fig. 4 of the gFC scenario. However, it is clear that they differ quite substantially from the allowed regions for the first and second generation in the remaining subfigures of Fig. 4.

\section{CONCLUSIONS}

In this paper, we analyze the question of general flavor conservation in extensions of the SM with additional scalar doublets, in particular the 2HDM. The effect of the one-loop renomalization group evolution of the Yukawa coupling matrices on $\mathrm{gFC}$ scenarios is discussed in detail. In particular, it is to be stressed that in the absence of Yukawa couplings with right-handed neutrinos gFC in the lepton sector is stable. For the quark sector, some one-loop RGE-stable scenarios are discussed, including the case of a Cabibbo-like quark mixing matrix. At a phenomenological level, we discuss the constraints that existing data on flavor-conserving processes, in particular, the ones related to the Higgs, impose on the parameters describing
$\mathrm{gFC}$ in the different fermion sectors, including a detailed numerical analysis of that parameter space. Direct $q \bar{q} \rightarrow \mathrm{h}$ production is also considered in detail; although it is completely negligible in the SM, that might not be the case in scenarios such as $2 \mathrm{HDMs}$, and it may even be relevant for the production of the additional non-SM neutral scalars.

\section{ACKNOWLEDGMENTS}

The authors thank Luca Fiorini for discussions. This work is partially supported by Spanish MINECO under Grants No. FPA2015-68318-R and No. FPA2017-85140C3-3-P and by the Severo Ochoa Excellence Center Project No. SEV-2014-0398, by Generalitat Valenciana under Grant No. GVPROMETEOII 2014-049 and by Fundação para a Ciência e a Tecnologia (FCT, Portugal) through Projects No. CERN/FIS-NUC/0010/ 2015 and No. CFTP-FCT Unit 777 (UID/FIS/00777/ 2013), which are partially funded through POCTI (FEDER), COMPETE, Quadro de Referencia Estrategico Nacional (QREN), and EU. M. N. acknowledges support from FCT through postdoctoral Grant No. SFRH/BPD/112999/2015.

\section{APPENDIX: RGE DETAILS}

The analysis of the RGE of the quark Yukawa couplings and the stability of the gFC scenario in Eq. (38) has been presented in detail for the set $\left\{\Gamma_{\alpha} \Gamma_{\beta}^{\dagger}\right\}$ in Secs. III A and III B. We reproduce in this Appendix the equations relevant for $\left\{\Gamma_{\alpha} \Gamma_{\beta}^{\dagger}\right\}$ and also for $\left\{\Gamma_{\alpha}^{\dagger} \Gamma_{\beta}\right\},\left\{\Delta_{\alpha} \Delta_{\beta}^{\dagger}\right\}$ and $\left\{\Delta_{\alpha}^{\dagger} \Delta_{\beta}\right\}$, omitted for conciseness in Sec. III. In correspondence with Eqs. (46)-(48),

$$
\begin{array}{ll}
\mathcal{D}\left(\Gamma_{\alpha} \Gamma_{\beta}^{\dagger}\right)=f_{\alpha \beta}^{\left[\mathrm{d}_{\mathrm{L}}\right]}(\Gamma)+g_{\alpha \beta}^{\left[\mathrm{d}_{\mathrm{L}}\right]}(\Gamma, \Delta), & \mathcal{D}\left(\Delta_{\alpha} \Delta_{\beta}^{\dagger}\right)=f_{\alpha \beta}^{\left[\mathrm{u}_{\mathrm{L}}\right]}(\Delta)+g_{\alpha \beta}^{\left[\mathrm{L}_{\mathrm{L}}\right]}(\Gamma, \Delta), \\
\mathcal{D}\left(\Gamma_{\alpha}^{\dagger} \Gamma_{\beta}\right)=f_{\alpha \beta}^{\left[\mathrm{d}_{\mathrm{R}}\right]}(\Gamma)+g_{\alpha \beta}^{\left[\mathrm{d}_{\mathrm{R}}\right]}(\Gamma, \Delta), & \mathcal{D}\left(\Delta_{\alpha}^{\dagger} \Delta_{\beta}\right)=f_{\alpha \beta}^{\left[\mathrm{u}_{\mathrm{R}}\right]}(\Delta)+g_{\alpha \beta}^{\left[\mathrm{u}_{\mathrm{R}}\right]}(\Gamma, \Delta),
\end{array}
$$

with

$$
\begin{gathered}
f_{\alpha \beta}^{\left[\mathrm{d}_{\mathrm{L}}\right]}(\Gamma)=2 a_{d} \Gamma_{\alpha} \Gamma_{\beta}^{\dagger}+\sum_{\rho=1}^{n=2}\left[T_{\alpha, \rho}^{d} \Gamma_{\rho} \Gamma_{\beta}^{\dagger}+T_{\beta, \rho}^{d *} \Gamma_{\alpha} \Gamma_{\rho}^{\dagger}\right]+2 \Gamma_{\alpha} \Gamma_{R} \Gamma_{\beta}^{\dagger}+\frac{1}{2} \Gamma_{L} \Gamma_{\alpha} \Gamma_{\beta}^{\dagger}+\frac{1}{2} \Gamma_{\alpha} \Gamma_{\beta}^{\dagger} \Gamma_{L}, \\
g_{\alpha \beta}^{\left[\mathrm{d}_{\mathrm{L}}\right]}(\Gamma, \Delta)=\frac{1}{2} \Delta_{L} \Gamma_{\alpha} \Gamma_{\beta}^{\dagger}+\frac{1}{2} \Gamma_{\alpha} \Gamma_{\beta}^{\dagger} \Delta_{L}-2 \sum_{\rho=1}^{n=2}\left[\Delta_{\rho} \Delta_{\alpha}^{\dagger} \Gamma_{\rho} \Gamma_{\beta}^{\dagger}+\Gamma_{\alpha} \Gamma_{\rho}^{\dagger} \Delta_{\beta} \Delta_{\rho}^{\dagger}\right], \\
f_{\alpha \beta}^{\left[\mathrm{d}_{\mathrm{R}}\right]}(\Gamma)=2 a_{d} \Gamma_{\alpha}^{\dagger} \Gamma_{\beta}+\sum_{\rho=1}^{n=2}\left[T_{\alpha, \rho}^{d *} \Gamma_{\rho}^{\dagger} \Gamma_{\beta}+T_{\beta, \rho}^{d} \Gamma_{\alpha}^{\dagger} \Gamma_{\rho}\right]+\Gamma_{\alpha}^{\dagger} \Gamma_{L} \Gamma_{\beta}+\Gamma_{R} \Gamma_{\alpha}^{\dagger} \Gamma_{\beta}+\Gamma_{\alpha}^{\dagger} \Gamma_{\beta} \Gamma_{R}, \\
g_{\alpha \beta}^{\left[\mathrm{d}_{R}\right]}(\Gamma, \Delta)=\Gamma_{\alpha}^{\dagger} \Delta_{L} \Gamma_{\beta}-2 \sum_{\rho=1}^{n=2}\left[\Gamma_{\rho}^{\dagger} \Delta_{\alpha} \Delta_{\rho}^{\dagger} \Gamma_{\beta}+\Gamma_{\alpha}^{\dagger} \Delta_{\rho} \Delta_{\beta}^{\dagger} \Gamma_{\rho}\right],
\end{gathered}
$$




$$
\begin{aligned}
f_{\alpha \beta}^{\left[\mathrm{u}_{\mathrm{L}}\right]}(\Delta) & =2 a_{u} \Delta_{\alpha} \Delta_{\beta}^{\dagger}+\sum_{\rho=1}^{n=2}\left[T_{\alpha, \rho}^{u} \Delta_{\rho} \Delta_{\beta}^{\dagger}+T_{\beta, \rho}^{d *} \Delta_{\alpha} \Delta_{\rho}^{\dagger}\right]+2 \Delta_{\alpha} \Delta_{R} \Delta_{\beta}^{\dagger}+\frac{1}{2} \Delta_{L} \Delta_{\alpha} \Delta_{\beta}^{\dagger}+\frac{1}{2} \Delta_{\alpha} \Delta_{\beta}^{\dagger} \Delta_{L}, \\
g_{\alpha \beta}^{\left[\mathrm{u}_{\mathrm{L}}\right]}(\Gamma, \Delta) & =\frac{1}{2} \Gamma_{L} \Delta_{\alpha} \Delta_{\beta}^{\dagger}+\frac{1}{2} \Delta_{\alpha} \Delta_{\beta}^{\dagger} \Gamma_{L}-2 \sum_{\rho=1}^{n=2}\left[\Gamma_{\rho} \Gamma_{\alpha}^{\dagger} \Delta_{\rho} \Delta_{\beta}^{\dagger}+\Delta_{\alpha} \Delta_{\rho}^{\dagger} \Gamma_{\beta} \Gamma_{\rho}^{\dagger}\right],
\end{aligned}
$$

and

$$
\begin{aligned}
f_{\alpha \beta}^{\left[\mathrm{u}_{\mathrm{R}}\right]}(\Gamma) & =2 a_{u} \Delta_{\alpha}^{\dagger} \Delta_{\beta}+\sum_{\rho=1}^{n=2}\left[T_{\alpha, \rho}^{u *} \Delta_{\rho}^{\dagger} \Delta_{\beta}+T_{\beta, \rho}^{u} \Delta_{\alpha}^{\dagger} \Delta_{\rho}\right]+\Delta_{\alpha}^{\dagger} \Delta_{L} \Delta_{\beta}+\Delta_{R} \Delta_{\alpha}^{\dagger} \Delta_{\beta}+\Delta_{\alpha}^{\dagger} \Delta_{\beta} \Delta_{R}, \\
g_{\alpha \beta}^{\left[\mathrm{u}_{\beta}\right]}(\Gamma, \Delta) & =\Delta_{\alpha}^{\dagger} \Gamma_{L} \Delta_{\beta}-2 \sum_{\rho=1}^{n=2}\left[\Delta_{\rho}^{\dagger} \Gamma_{\alpha} \Gamma_{\rho}^{\dagger} \Delta_{\beta}+\Delta_{\alpha}^{\dagger} \Gamma_{\rho} \Gamma_{\beta}^{\dagger} \Delta_{\rho}\right] .
\end{aligned}
$$

The RGE of the commutation relations of Eq. (37) reads

$$
\begin{aligned}
\mathcal{D}\left[\Gamma_{\alpha} \Gamma_{\beta}^{\dagger}, \Gamma_{\gamma} \Gamma_{\delta}^{\dagger}\right] & =\left[g_{\alpha \beta}^{\left[\mathrm{d}_{\mathrm{L}}\right]}(\Gamma, \Delta), \Gamma_{\gamma} \Gamma_{\delta}^{\dagger}\right]+\left[\Gamma_{\alpha} \Gamma_{\beta}^{\dagger}, g_{\gamma \delta}^{\left[\mathrm{d}_{\mathrm{L}}\right]}(\Gamma, \Delta)\right], \\
\mathcal{D}\left[\Gamma_{\alpha}^{\dagger} \Gamma_{\beta}, \Gamma_{\gamma}^{\dagger} \Gamma_{\delta}\right] & =\left[g_{\alpha \beta}^{\left[\mathrm{d}_{\mathrm{R}}\right]}(\Gamma, \Delta), \Gamma_{\gamma}^{\dagger} \Gamma_{\delta}\right]+\left[\Gamma_{\alpha}^{\dagger} \Gamma_{\beta}, g_{\gamma \delta}^{\left[\mathrm{d}_{\mathrm{R}}\right]}(\Gamma, \Delta)\right], \\
\mathcal{D}\left[\Delta_{\alpha} \Delta_{\beta}^{\dagger}, \Delta_{\gamma} \Delta_{\delta}^{\dagger}\right] & =\left[g_{\alpha \beta}^{\left[\mathrm{u}_{\mathrm{L}}\right]}(\Gamma, \Delta), \Delta_{\gamma} \Delta_{\delta}^{\dagger}\right]+\left[\Delta_{\alpha} \Delta_{\beta}^{\dagger}, g_{\gamma \delta}^{\left[\mathrm{u}_{\mathrm{L}}\right]}(\Gamma, \Delta)\right], \\
\mathcal{D}\left[\Delta_{\alpha}^{\dagger} \Delta_{\beta}, \Delta_{\gamma}^{\dagger} \Delta_{\delta}\right] & =\left[g_{\alpha \beta}^{\left[\mathrm{u}_{\mathrm{R}}\right]}(\Gamma, \Delta), \Delta_{\gamma}^{\dagger} \Gamma_{\delta}\right]+\left[\Delta_{\alpha}^{\dagger} \Delta_{\beta}, g_{\gamma \delta}^{\left[\mathrm{u}_{\mathrm{R}}\right]}(\Gamma, \Delta)\right],
\end{aligned}
$$

which, following the discussion in Sec. III B, lead to (summation over $h=1,2$ understood)

$$
\begin{aligned}
& \left\{d_{L}\right\} \equiv \frac{v^{2}}{2} U_{\mathrm{d}_{L}}^{\dagger}\left(\mathcal{D}\left[Y_{[\mathrm{d}] l}^{0} Y_{[\mathrm{d}] j}^{0^{\dagger}}, Y_{[\mathrm{d}] k}^{0} Y_{[\mathrm{d}] l}^{0^{\dagger}}\right]\right) U_{\mathrm{d}_{L}} \\
& =Y_{[\mathrm{d}] i} Y_{[\mathrm{d}] j}^{\dagger} V^{\dagger} Y_{[\mathrm{u}] h} Y_{[\mathrm{u}] h}^{\dagger} V Y_{[\mathrm{d}] k} Y_{[\mathrm{d}] l}^{\dagger}-Y_{[\mathrm{d}] k} Y_{[\mathrm{d}] l}^{\dagger} V^{\dagger} Y_{[\mathrm{u}] h} Y_{[\mathrm{u}] h}^{\dagger} V Y_{[\mathrm{d}] i} Y_{[\mathrm{d}] j}^{\dagger} \\
& -2\left[V^{\dagger} Y_{[\mathrm{u}] h} Y_{[\mathrm{u}] i}^{\dagger} V, Y_{[\mathrm{d}] k} Y_{[\mathrm{d}] l}^{\dagger}\right] Y_{[\mathrm{d}] h} Y_{[\mathrm{d}] j}^{\dagger}-2 Y_{[\mathrm{d}] i} Y_{[\mathrm{d}] h}^{\dagger}\left[V^{\dagger} Y_{[\mathrm{u}] j} Y_{[\mathrm{u}] h}^{\dagger} V, Y_{[\mathrm{d}] k} Y_{[\mathrm{d}] l}^{\dagger}\right] \\
& +2\left[V^{\dagger} Y_{[\mathrm{u}] h} Y_{[\mathrm{u}] k}^{\dagger} V, Y_{[\mathrm{d}] i} Y_{[\mathrm{d}] j}^{\dagger}\right] Y_{[\mathrm{d}] h} Y_{[\mathrm{d}] l}^{\dagger}+2 Y_{[\mathrm{d}] k} Y_{[\mathrm{d}] h}^{\dagger}\left[V^{\dagger} Y_{[\mathrm{u}] l} Y_{[\mathrm{u}] h}^{\dagger} V, Y_{[\mathrm{d}] i} Y_{[\mathrm{d}] j}^{\dagger}\right], \\
& \left\{d_{R}\right\} \equiv \frac{v^{2}}{2} U_{\mathrm{d}_{R}}^{\dagger}\left(\mathcal{D}\left[Y_{[\mathrm{d}] i}^{0 \dagger} Y_{[\mathrm{d}] j}^{0}, Y_{[\mathrm{d}] k}^{0 \dagger} Y_{[\mathrm{d}]}^{0}\right]\right) U_{\mathrm{d}_{R}} \\
& =\left[Y_{[\mathrm{d}] i}^{\dagger} V^{\dagger} Y_{[\mathrm{u}] h} Y_{[\mathrm{u}] h}^{\dagger} V Y_{[\mathrm{d}] j}, Y_{[\mathrm{d}] k}^{\dagger} Y_{[\mathrm{d}] l}\right]-\left[Y_{[\mathrm{d}] k}^{\dagger} V^{\dagger} Y_{[\mathrm{u}] h} Y_{[\mathrm{u}] h}^{\dagger} V Y_{[\mathrm{d}] l}, Y_{[\mathrm{d}] i}^{\dagger} Y_{[\mathrm{d}] j}\right] \\
& -2\left[Y_{[\mathrm{d}] h}^{\dagger} V^{\dagger} Y_{[\mathrm{u}] i} Y_{[\mathrm{u}] h}^{\dagger} V Y_{[\mathrm{d}] j}, Y_{[\mathrm{d}] k}^{\dagger} Y_{[\mathrm{d}] l}\right]-2\left[Y_{[\mathrm{d}] i}^{\dagger} V^{\dagger} Y_{[\mathrm{u}] h} Y_{[\mathrm{u}] j}^{\dagger} V Y_{[\mathrm{d}] h}, Y_{[\mathrm{d}] k}^{\dagger} Y_{[\mathrm{d}] l}\right] \\
& +2\left[Y_{[\mathrm{d}] h}^{\dagger} V^{\dagger} Y_{[\mathrm{u}] k} Y_{[\mathrm{u}] h}^{\dagger} V Y_{[\mathrm{d}] l}, Y_{[\mathrm{d}] i}^{\dagger} Y_{[\mathrm{d}] j}\right]+2\left[Y_{[\mathrm{d}] k}^{\dagger} V^{\dagger} Y_{[\mathrm{u}] h} Y_{[\mathrm{u}] l}^{\dagger} V Y_{[\mathrm{d}] h}, Y_{[\mathrm{d}] i}^{\dagger} Y_{[\mathrm{d}] j}\right], \\
& \left\{u_{L}\right\} \equiv \frac{v^{2}}{2} U_{\mathrm{u}_{L}}^{\dagger}\left(\mathcal{D}\left[Y_{[\mathrm{u}] i}^{0} Y_{[\mathrm{u}] j}^{0 \dagger}, Y_{[\mathrm{u}] k}^{0} Y_{[\mathrm{u}] l}^{0 \dagger}\right]\right) U_{\mathrm{u}_{L}} \\
& =Y_{[\mathrm{u}] i} Y_{[\mathrm{u}] j}^{\dagger} V Y_{[\mathrm{d}] h} Y_{[\mathrm{d}] h}^{\dagger} V^{\dagger} Y_{[\mathrm{u}] k} Y_{[\mathrm{u}] l}^{\dagger}-Y_{[\mathrm{u}] k} Y_{[\mathrm{u}] l}^{\dagger} V Y_{[\mathrm{d}] h} Y_{[\mathrm{d}] h}^{\dagger} V^{\dagger} Y_{[\mathrm{u}] i} Y_{[\mathrm{u}] j}^{\dagger} \\
& -2\left[V Y_{[\mathrm{d}] h} Y_{[\mathrm{d}] i}^{\dagger} V^{\dagger}, Y_{[\mathrm{u}] k} Y_{[\mathrm{u}] l}^{\dagger}\right] Y_{[\mathrm{u}] h} Y_{[\mathrm{u}] j}^{\dagger}-2 Y_{[\mathrm{u}] i} Y_{[\mathrm{u}] h}^{\dagger}\left[V Y_{[\mathrm{d}] j} Y_{[\mathrm{d}] h}^{\dagger} V^{\dagger}, Y_{[\mathrm{u}] k} Y_{[\mathrm{u}] l}^{\dagger}\right] \\
& +2\left[V Y_{[\mathrm{d}] h} Y_{[\mathrm{d}] k}^{\dagger} V^{\dagger}, Y_{[\mathrm{u}]} Y_{[\mathrm{u}] j}^{\dagger}\right] Y_{[\mathrm{u}] h} Y_{[\mathrm{u}] l}^{\dagger}+2 Y_{[\mathrm{u}] k} Y_{[\mathrm{u}] h}^{\dagger}\left[V Y_{[\mathrm{d}] l} Y_{[\mathrm{d}] h}^{\dagger} V^{\dagger}, Y_{[\mathrm{u}] i} Y_{[\mathrm{u}] j}^{\dagger}\right],
\end{aligned}
$$


and

$$
\begin{aligned}
\left\{u_{R}\right\} \equiv & \frac{v^{2}}{2} U_{\mathrm{u} R}^{\dagger}\left(\mathcal{D}\left[Y_{[\mathrm{u}] i}^{0 \dagger} Y_{[\mathrm{u}] j}^{0}, Y_{[\mathrm{u}] k}^{0 \dagger} Y_{[\mathrm{u}] l}^{0}\right]\right) U_{\mathrm{u}_{R}} \\
= & {\left[Y_{[\mathrm{u}]}^{\dagger} V Y_{[\mathrm{d}] h} Y_{[\mathrm{d}] h}^{\dagger} V^{\dagger} Y_{[\mathrm{u}] j}, Y_{[\mathrm{u}] k}^{\dagger} Y_{[\mathrm{u}] l}\right]-\left[Y_{[\mathrm{u}] k}^{\dagger} V Y_{[\mathrm{d}] h} Y_{[\mathrm{d}] h}^{\dagger} V^{\dagger} Y_{[\mathrm{u}] l}, Y_{[\mathrm{u}] i}^{\dagger} Y_{[\mathrm{u}] j}\right] } \\
& -2\left[Y_{[\mathrm{u}] h}^{\dagger} V Y_{[\mathrm{d}] l} Y_{[\mathrm{d}] h}^{\dagger} V^{\dagger} Y_{[\mathrm{u}] j}, Y_{[\mathrm{u}] k}^{\dagger} Y_{[\mathrm{u}] l}\right]-2\left[Y_{[\mathrm{u}]}^{\dagger} V Y_{[\mathrm{d}] h} Y_{[\mathrm{d}] j}^{\dagger} V^{\dagger} Y_{[\mathrm{u}] h}, Y_{[\mathrm{u}] k}^{\dagger} Y_{[\mathrm{u}] l}\right] \\
& +2\left[Y_{[\mathrm{u}] h}^{\dagger} V Y_{[\mathrm{d}] k} Y_{[\mathrm{d}] h}^{\dagger} V^{\dagger} Y_{[\mathrm{u}] l}, Y_{[\mathrm{u}] i}^{\dagger} Y_{[\mathrm{u}] j}\right]+2\left[Y_{[\mathrm{u}] k}^{\dagger} V Y_{[\mathrm{d}] h} Y_{[\mathrm{d}] l}^{\dagger} V^{\dagger} Y_{[\mathrm{u}] h}, Y_{[\mathrm{u}] i}^{\dagger} Y_{[\mathrm{u}] j}\right] .
\end{aligned}
$$

To compute the matrix elements of Eqs. (A7)-(A10), we notice that

$$
\left.\left(\left[V^{\dagger} Y_{[\mathrm{u}] i} Y_{[\mathrm{u}] j}^{\dagger} V, Y_{[\mathrm{d}] k}^{\dagger} Y_{[\mathrm{d}]}\right]\right]\right)_{a b}=\sum_{q=1}^{3} V_{q a}^{*} V_{q b} y_{i, q}^{\mathrm{u}} y_{j, q}^{\mathrm{u} *}\left(y_{k, b}^{\mathrm{d} *} y_{l, b}^{\mathrm{d}}-y_{k, a}^{\mathrm{d} *} y_{l, a}^{\mathrm{d}}\right),
$$

and

$$
\left(\left[V Y_{[\mathrm{d}] i} Y_{[\mathrm{d}] j}^{\dagger} V^{\dagger}, Y_{[\mathrm{u}] k} Y_{[\mathrm{u}]}^{\dagger}\right]\right)_{a b}=\sum_{q=1}^{3} V_{a q} V_{b q}^{*} y_{i, q}^{\mathrm{d}} y_{j, q}^{\mathrm{d} *}\left(y_{k, b}^{\mathrm{u}} y_{l, b}^{\mathrm{u} *}-y_{k, a}^{\mathrm{u}} y_{l, a}^{\mathrm{u} *}\right) .
$$

Then, with the parameters in Eq. (62), the matrix elements $(a, b)$ of Eqs. (A7)-(A10) read

$$
\begin{aligned}
\left\{d_{L}\right\}_{a b}= & \sum_{q=1}^{3} \sum_{\mathrm{h}=1}^{n=2} V_{q a}^{*} V_{q b}\left\{\left|y_{h, q}^{\mathrm{u}}\right|^{2}\left(y_{i, a}^{\mathrm{d}} y_{j, a}^{\mathrm{d} *} y_{k, b}^{\mathrm{d}} y_{l, b}^{\mathrm{d} *}-y_{i, b}^{\mathrm{d}} y_{j, b}^{\mathrm{d} *} y_{k, a}^{\mathrm{d}} y_{l, a}^{\mathrm{d} *}\right)\right. \\
& -2\left(y_{h, q}^{\mathrm{u}} y_{i, q}^{\mathrm{u} *} y_{h, b}^{\mathrm{d}} y_{j, b}^{\mathrm{d} *}+y_{j, q}^{\mathrm{u}} y_{h, q}^{\mathrm{u} *} y_{i, a}^{\mathrm{d}} y_{h, a}^{\mathrm{d} *}\right)\left(y_{k, b}^{\mathrm{d}} y_{l, b}^{\mathrm{d} *}-y_{k, a}^{\mathrm{d}} y_{l, a}^{\mathrm{d} *}\right) \\
& \left.+2\left(y_{h, q}^{\mathrm{u}} y_{k, q}^{\mathrm{u} *} y_{h, b}^{\mathrm{d}} y_{l, b}^{\mathrm{d} *}+y_{l, q}^{\mathrm{u}} y_{h, q}^{\mathrm{u} *} y_{k, a}^{\mathrm{d}} y_{h, a}^{\mathrm{d} *}\right)\left(y_{i, b}^{\mathrm{d}} y_{j, b}^{\mathrm{d} *}-y_{i, a}^{\mathrm{d}} y_{j, a}^{\mathrm{d} *}\right)\right\} . \\
\left\{d_{R}\right\}_{a b}= & \sum_{q=1}^{3} \sum_{\mathrm{h}=1}^{n=2} V_{q a}^{*} V_{q b}\left\{\left(y_{k, b}^{\mathrm{d} *} y_{l, b}^{\mathrm{d}}-y_{k, a}^{\mathrm{d} *} y_{l, a}^{\mathrm{d}}\right)\left(\left|y_{h, q}^{\mathrm{u}}\right|^{2} y_{i, a}^{\mathrm{d} *} y_{j, b}^{\mathrm{d}}-2 y_{i, q}^{\mathrm{u}} y_{h, q}^{\mathrm{u} *} y_{h, a}^{\mathrm{d} *} y_{j, b}^{\mathrm{d}}-2 y_{h, q}^{\mathrm{u}} y_{j, q}^{\mathrm{u} *} y_{i, a}^{\mathrm{d} *} y_{h, b}^{\mathrm{d}}\right)\right. \\
& \left.-\left(y_{i, b}^{\mathrm{d} *} y_{j, b}^{\mathrm{d}}-y_{i, a}^{\mathrm{d} *} y_{j, a}^{\mathrm{d}}\right)\left(\left|y_{h, q}^{\mathrm{u}}\right|^{2} y_{k, a}^{\mathrm{d} *} y_{l, b}^{\mathrm{d}}-2 y_{k, q}^{\mathrm{u}} y_{h, q}^{\mathrm{u} *} y_{h, a}^{\mathrm{d} *} y_{l, b}^{\mathrm{d}}-2 y_{h, q}^{\mathrm{u}} y_{l, q}^{\mathrm{u} *} y_{k, a}^{\mathrm{d} *} y_{h, b}^{\mathrm{d}}\right)\right\}, \\
\left\{u_{L}\right\}_{a b}= & \sum_{q=1}^{3} \sum_{\mathrm{h}=1}^{n=2} V_{a q} V_{b q}^{*}\left\{\left|y_{h, q}^{\mathrm{d}}\right|^{2}\left(y_{i, a}^{\mathrm{u}} y_{j, a}^{\mathrm{u} *} y_{k, b}^{\mathrm{u}} y_{l, b}^{\mathrm{u} *}-y_{i, b}^{\mathrm{u}} y_{j, b}^{\mathrm{u} *} y_{k, a}^{\mathrm{u}} y_{l, a}^{\mathrm{u} *}\right)\right. \\
& -2\left(y_{h, q}^{\mathrm{d}} y_{i, q}^{\mathrm{d} *} y_{h, b}^{\mathrm{u}} y_{j, b}^{\mathrm{u} *}+y_{j, q}^{\mathrm{d}} y_{h, q}^{\mathrm{d} *} y_{i, a}^{\mathrm{u}} y_{h, a}^{\mathrm{u} *}\right)\left(y_{k, b}^{\mathrm{u}} y_{l, b}^{\mathrm{u} *}-y_{k, a}^{\mathrm{u}} y_{l, a}^{\mathrm{u} *}\right) \\
& \left.+2\left(y_{h, q}^{\mathrm{d}} y_{k, q}^{\mathrm{d} *} y_{h, b}^{\mathrm{u}} y_{l, b}^{\mathrm{u} *}+y_{l, q}^{\mathrm{d}} y_{h, q}^{\mathrm{d} *} y_{k, a}^{\mathrm{u}} y_{h, a}^{\mathrm{u} *}\right)\left(y_{i, b}^{\mathrm{u}} y_{j, b}^{\mathrm{u} *}-y_{i, a}^{\mathrm{u}} y_{j, a}^{\mathrm{u} *}\right)\right\},
\end{aligned}
$$

and

$$
\begin{aligned}
\left\{u_{R}\right\}_{a b}= & \sum_{q=1}^{3} \sum_{\mathrm{h}=1}^{n=2} V_{a q} V_{b q}^{*}\left\{\left(y_{k, b}^{\mathrm{u} *} y_{l, b}^{\mathrm{u}}-y_{k, a}^{\mathrm{u} *} y_{l, a}^{\mathrm{u}}\right)\left(\left|y_{h, q}^{\mathrm{d}}\right|^{2} y_{i, a}^{\mathrm{u} *} y_{j, b}^{\mathrm{u}}-2 y_{i, q}^{\mathrm{d}} y_{h, q}^{\mathrm{d} *} y_{h, a}^{\mathrm{u} *} y_{j, b}^{\mathrm{u}}-2 y_{h, q}^{\mathrm{d}} y_{j, q}^{\mathrm{d} *} y_{i, a}^{\mathrm{u} *} y_{h, b}^{\mathrm{u}}\right)\right. \\
& \left.-\left(y_{i, b}^{\mathrm{u} *} y_{j, b}^{\mathrm{u}}-y_{i, a}^{\mathrm{u} *} y_{j, a}^{\mathrm{u}}\right)\left(\left|y_{h, q}^{\mathrm{d}}\right|^{2} y_{k, a}^{\mathrm{u} *} y_{l, b}^{\mathrm{u}}-2 y_{k, q}^{\mathrm{d}} y_{h, q}^{\mathrm{d} *} y_{h, a}^{\mathrm{u} *} y_{l, b}^{\mathrm{u}}-2 y_{h, q}^{\mathrm{d}} y_{l, q}^{\mathrm{d} *} y_{k, a}^{\mathrm{u} *} y_{h, b}^{\mathrm{u}}\right)\right\} .
\end{aligned}
$$

For diagonal elements, $a=b$, the right-hand sides of Eqs. (A13)-(A16) are identically zero. For $i=j$ and $k=l$, by construction, we have in addition $\left\{q_{X}\right\}_{b a}=-\left\{q_{X}\right\}_{a b}^{*}(q=u, d, X=L, R)$. For illustration, we show in the following Eqs. (A13)-(A16) for 2HDM and $i=j=1, k=l=2$ : 


$$
\begin{aligned}
& \left\{d_{L}\right\}_{d_{a} d_{b}}=\sum_{u_{q}=1}^{3} V_{u_{q} d_{a}}^{*} V_{u_{q} d_{b}}\left\{\left(m_{u_{q}}^{2}+\left|n_{u_{q}}\right|^{2}\right)\left(m_{d_{a}}^{2}\left|n_{d_{b}}\right|^{2}-m_{d_{b}}^{2}\left|n_{d_{a}}\right|^{2}\right)-2\left(\left|n_{d_{b}}\right|^{2}-\left|n_{d_{a}}\right|^{2}\right)\left(m_{u_{q}} m_{d_{b}}\left(m_{u_{q}} m_{d_{b}}+n_{u_{q}} n_{d_{b}}\right)\right.\right. \\
& \left.\left.+m_{u_{q}} m_{d_{a}}\left(m_{u_{q}} m_{d_{a}}+n_{u_{q}}^{*} n_{d_{a}}^{*}\right)\right)+2\left(m_{d_{b}}^{2}-m_{d_{a}}^{2}\right)\left(n_{u_{q}}^{*} n_{d_{b}}^{*}\left(m_{u_{q}} m_{d_{b}}+n_{u_{q}} n_{d_{b}}\right)+n_{u_{q}} n_{d_{a}}\left(m_{u_{q}} m_{d_{a}}+n_{u_{q}}^{*} n_{d_{a}}^{*}\right)\right)\right\}, \\
& \left\{d_{R}\right\}_{d_{a} d_{b}}=\sum_{u_{q}=1}^{3} V_{u_{q} d_{a}}^{*} V_{u_{q} d_{b}}\left\{\left(\left|n_{d_{b}}\right|^{2}-\left|n_{d_{a}}\right|^{2}\right)\left(m_{u_{q}}^{2}+\left|n_{u_{q}}\right|^{2}\right) m_{d_{a}} m_{d_{b}}-2\left(\left|n_{d_{b}}\right|^{2}-\left|n_{d_{a}}\right|^{2}\right)\left(m_{u_{q}} m_{d_{b}}\left(m_{u_{q}} m_{d_{a}}+n_{u_{q}}^{*} n_{d_{a}}^{*}\right)\right.\right. \\
& \left.+m_{u_{q}} m_{d_{a}}\left(m_{u_{q}} m_{d_{b}}+n_{u_{q}} n_{d_{b}}\right)\right)-\left(m_{d_{b}}^{2}-m_{d_{a}}^{2}\right)\left(m_{u_{q}}^{2}+\left|n_{u_{q}}\right|^{2}\right) n_{d_{a}}^{*} n_{d_{b}} \\
& \left.+2\left(m_{d_{b}}^{2}-m_{d_{a}}^{2}\right)\left(n_{u_{q}} n_{d_{b}}\left(m_{u_{q}} m_{d_{a}}+n_{u_{q}}^{*} n_{d_{a}}^{*}\right)+n_{u_{q}}^{*} n_{d_{a}}^{*}\left(m_{u_{q}} m_{d_{b}}+n_{u_{q}} n_{d_{b}}\right)\right)\right\} \\
& \left\{u_{L}\right\}_{u_{a} u_{b}}=\sum_{d_{q}=1}^{3} V_{u_{a} d_{q}} V_{u_{b} d_{q}}^{*}\left\{\left(m_{d_{q}}^{2}+\left|n_{d_{q}}\right|^{2}\right)\left(m_{u_{a}}^{2}\left|n_{u_{b}}\right|^{2}-m_{u_{b}}^{2}\left|n_{u_{a}}\right|^{2}\right)-2\left(\left|n_{u_{b}}\right|^{2}-\left|n_{u_{a}}\right|^{2}\right)\left(m_{d_{q}} m_{u_{b}}\left(m_{d_{q}} m_{u_{b}}+n_{d_{q}} n_{u_{b}}\right)\right.\right. \\
& \left.\left.+m_{d_{q}} m_{u_{a}}\left(m_{d_{q}} m_{u_{a}}+n_{d_{q}}^{*} n_{u_{a}}^{*}\right)\right)+2\left(m_{u_{b}}^{2}-m_{u_{a}}^{2}\right)\left(n_{d_{q}}^{*} n_{u_{b}}^{*}\left(m_{d_{q}} m_{u_{b}}+n_{d_{q}} n_{u_{b}}\right)+n_{d_{q}} n_{u_{a}}\left(m_{d_{q}} m_{u_{a}}+n_{d_{q}}^{*} n_{u_{a}}^{*}\right)\right)\right\},
\end{aligned}
$$

and

$$
\begin{aligned}
\left\{u_{R}\right\}_{u_{a} u_{b}}= & \sum_{q=1}^{3} V_{u_{a} d_{q}} V_{u_{b} d_{q}}^{*}\left\{\left(\left|n_{u_{b}}\right|^{2}-\left|n_{u_{a}}\right|^{2}\right)\left(m_{d_{q}}^{2}+\left|n_{d_{q}}\right|^{2}\right) m_{u_{a}} m_{u_{b}}-2\left(\left|n_{u_{b}}\right|^{2}-\left|n_{u_{a}}\right|^{2}\right)\left(m_{d_{q}} m_{u_{b}}\left(m_{d_{q}} m_{u_{a}}+n_{d_{q}}^{*} n_{u_{a}}^{*}\right)\right.\right. \\
& \left.+m_{d_{q}} m_{u_{a}}\left(m_{d_{q}} m_{u_{b}}+n_{d_{q}} n_{u_{b}}\right)\right)-\left(m_{u_{b}}^{2}-m_{u_{a}}^{2}\right)\left(m_{d_{q}}^{2}+\left|n_{d_{q}}\right|^{2}\right) n_{u_{a}}^{*} n_{u_{b}} \\
& \left.+2\left(m_{u_{b}}^{2}-m_{u_{a}}^{2}\right)\left(n_{d_{q}} n_{u_{b}}\left(m_{d_{q}} m_{u_{a}}+n_{d_{q}}^{*} n_{u_{a}}^{*}\right)+n_{d_{q}}^{*} n_{u_{a}}^{*}\left(m_{d_{q}} m_{u_{b}}+n_{d_{q}} n_{u_{b}}\right)\right)\right\} .
\end{aligned}
$$

The formal generalization of the conditions in this Appendix and in Sec. III to the case of models with $n$ Higgs doublets instead of two is almost straightforward.

[1] T. Lee, Phys. Rev. D 8, 1226 (1973).

[2] G. Branco, P. M. Ferreira, L. Lavoura, M. N. Rebelo, M. Sher, and J. P. Silva, Phys. Rep. 516, 1 (2012).

[3] I. P. Ivanov, Prog. Part. Nucl. Phys. 95, 160 (2017).

[4] G. Aad et al. (ATLAS Collaboration), Phys. Lett. B 716, 1 (2012).

[5] S. Chatrchyan et al. (CMS Collaboration), Phys. Lett. B 716, 30 (2012).

[6] H. E. Haber, G. L. Kane, and T. Sterling, Nucl. Phys. B161, 493 (1979).

[7] J. F. Donoghue and L.F. Li, Phys. Rev. D 19, 945 (1979).

[8] L. F. Abbott, P. Sikivie, and M. B. Wise, Phys. Rev. D 21, 1393 (1980).

[9] L. J. Hall and M. B. Wise, Nucl. Phys. B187, 397 (1981).

[10] V. D. Barger, J. L. Hewett, and R. J. N. Phillips, Phys. Rev. D 41, 3421 (1990).

[11] D. Atwood, L. Reina, and A. Soni, Phys. Rev. D 55, 3156 (1997).
[12] A. Wahab El Kaffas, P. Osland, and O. M. Ogreid, Phys. Rev. D 76, 095001 (2007).

[13] M. Aoki, S. Kanemura, K. Tsumura, and K. Yagyu, Phys. Rev. D 80, 015017 (2009).

[14] F. Mahmoudi and O. Stal, Phys. Rev. D 81, 035016 (2010).

[15] O. Deschamps, S. Descotes-Genon, S. Monteil, V. Niess, S. T'Jampens, and V. Tisserand, Phys. Rev. D 82, 073012 (2010).

[16] A. Crivellin, A. Kokulu, and C. Greub, Phys. Rev. D 87, 094031 (2013).

[17] A. Broggio, E. J. Chun, M. Passera, K. M. Patel, and S. K. Vempati, J. High Energy Phys. 11 (2014) 058.

[18] D. Das, Int. J. Mod. Phys. A 30, 1550158 (2015).

[19] R. Gaitán, R. Martinez, and J. H. M. de Oca, Phys. Rev. D 94, 094038 (2016).

[20] B. Altunkaynak, W.-S. Hou, C. Kao, M. Kohda, and B. McCoy, Phys. Lett. B 751, 135 (2015).

[21] A. Arhrib, R. Benbrik, C.-H. Chen, M. Gomez-Bock, and S. Semlali, Eur. Phys. J. C 76, 328 (2016). 
[22] C. S. Kim, Y. W. Yoon, and X.-B. Yuan, J. High Energy Phys. 12 (2015) 038.

[23] T. Enomoto and R. Watanabe, J. High Energy Phys. 05 (2016) 002.

[24] R. Benbrik, C.-H. Chen, and T. Nomura, Phys. Rev. D 93, 095004 (2016).

[25] J. M. Cline, Phys. Rev. D 93, 075017 (2016).

[26] L. Wang, F. Zhang, and X.-F. Han, Phys. Rev. D 95, 115014 (2017).

[27] S. Gori, H. E. Haber, and E. Santos, J. High Energy Phys. 06 (2017) 110.

[28] A. Arbey, F. Mahmoudi, O. Stal, and T. Stefaniak, Eur. Phys. J. C 78, 182 (2018).

[29] N. G. Deshpande and E. Ma, Phys. Rev. D 18, 2574 (1978).

[30] L. L. Honorez, E. Nezri, J. F. Oliver, and M. H. G. Tytgat, J. Cosmol. Astropart. Phys. 02 (2007) 028.

[31] M. Bona et al. (UTfit Collaboration), J. High Energy Phys. 07 (2005) 028.

[32] J. Charles, A. Hocker, H. Lacker, S. Laplace, F. R. Le Diberder, J. Malcles, J. Ocariz, M. Pivk, and L. Roos (CKMfitter Group Collaboration), Eur. Phys. J. C 41, 1 (2005).

[33] F. Botella, G. Branco, M. Nebot, and M. Rebelo, Nucl. Phys. B725, 155 (2005).

[34] N. Turok and J. Zadrozny, Nucl. Phys. B358, 471 (1991).

[35] H.-K. Guo, Y.-Y. Li, T. Liu, M. Ramsey-Musolf, and J. Shu, Phys. Rev. D 96, 115034 (2017).

[36] K. Fuyuto, W.-S. Hou, and E. Senaha, Phys. Lett. B 776, 402 (2018).

[37] H. Georgi and D. V. Nanopoulos, Phys. Lett. 82B, 95 (1979).

[38] S. L. Glashow and S. Weinberg, Phys. Rev. D 15, 1958 (1977).

[39] E. Paschos, Phys. Rev. D 15, 1966 (1977).

[40] P. Ko, Y. Omura, and C. Yu, Phys. Lett. B 717, 202 (2012).

[41] M. D. Campos, D. Cogollo, M. Lindner, T. Melo, F. S. Queiroz, and W. Rodejohann, J. High Energy Phys. 08 (2017) 092.

[42] R. Gatto, G. Morchio, and F. Strocchi, Phys. Lett. 80B, 265 (1979).

[43] R. Gatto, G. Morchio, G. Sartori, and F. Strocchi, Nucl. Phys. B163, 221 (1980).

[44] G. Sartori, Phys. Lett. 82B, 255 (1979).

[45] W. Grimus and G. Ecker, J. Phys. A 19, 3917 (1986).

[46] R. Barbieri, R. Gatto, and F. Strocchi, Phys. Lett. 74B, 344 (1978).

[47] G. Sartori, Nuovo Cimento A 55, 377 (1980).

[48] R. Gatto, G. Morchio, and F. Strocchi, Phys. Lett. 83B, 348 (1979).

[49] G. Segre and H. A. Weldon, Phys. Lett. 86B, 291 (1979).

[50] G. Segre and H. A. Weldon, Ann. Phys. (N.Y.) 124, 37 (1980).

[51] A. C. Rothman and K. Kang, Phys. Rev. D 23, 2657 (1981).

[52] K. Kang and A. C. Rothman, Phys. Rev. D 24, 167 (1981).

[53] M. Leurer, Y. Nir, and N. Seiberg, Nucl. Phys. B398, 319 (1993).

[54] T. P. Cheng and M. Sher, Phys. Rev. D 35, 3484 (1987).
[55] A. Antaramian, L. J. Hall, and A. Rasin, Phys. Rev. Lett. 69, 1871 (1992).

[56] L. J. Hall and S. Weinberg, Phys. Rev. D 48, R979 (1993).

[57] Y. L. Wu and L. Wolfenstein, Phys. Rev. Lett. 73, 1762 (1994).

[58] A. Datta, Phys. Rev. D 78, 095004 (2008).

[59] A. S. Joshipura, Mod. Phys. Lett. A 06, 1693 (1991).

[60] A. S. Joshipura and S. D. Rindani, Phys. Lett. B 260, 149 (1991).

[61] A. S. Joshipura and B. P. Kodrani, Phys. Rev. D 77, 096003 (2008).

[62] A. S. Joshipura and B. P. Kodrani, Phys. Rev. D 82, 115013 (2010).

[63] L. Lavoura, Int. J. Mod. Phys. A 09, 1873 (1994).

[64] G. Branco, W. Grimus, and L. Lavoura, Phys. Lett. B 380, 119 (1996).

[65] F. Botella, G. Branco, and M. Rebelo, Phys. Lett. B 687, 194 (2010).

[66] F. Botella, G. Branco, M. Nebot, and M. Rebelo, J. High Energy Phys. 10 (2011) 037.

[67] F. J. Botella, G. C. Branco, M. Nebot, and M. N. Rebelo, Eur. Phys. J. C 76, 161 (2016).

[68] J. M. Alves, F. J. Botella, G. C. Branco, F. Cornet-Gomez, and M. Nebot, Eur. Phys. J. C 77, 585 (2017).

[69] A. Pich and P. Tuzon, Phys. Rev. D 80, 091702 (2009).

[70] G. Ecker, W. Grimus, and H. Neufeld, Phys. Lett. B 228, 401 (1989).

[71] H. Serodio, Phys. Lett. B 700, 133 (2011).

[72] I. de Medeiros Varzielas, Phys. Lett. B 701, 597 (2011).

[73] M. B. Wise, Phys. Lett. 103B, 121 (1981).

[74] J. M. Frere and Y.-P. Yao, Phys. Rev. Lett. 55, 2386 (1985).

[75] G. Cvetic, S. S. Hwang, and C. S. Kim, Int. J. Mod. Phys. A 14, 769 (1999).

[76] G. Cvetic, C. S. Kim, and S. S. Hwang, Phys. Rev. D 58, 116003 (1998).

[77] P. Ferreira, L. Lavoura, and J. P. Silva, Phys. Lett. B 688, 341 (2010).

[78] J. Bijnens, J. Lu, and J. Rathsman, J. High Energy Phys. 05 (2012) 118.

[79] F. J. Botella, G. C. Branco, A. M. Coutinho, M. N. Rebelo, and J. I. Silva-Marcos, Eur. Phys. J. C 75, 286 (2015).

[80] A. Peñuelas and A. Pich, J. High Energy Phys. 12 (2017) 084.

[81] Y. H. Ahn and C.-H. Chen, Phys. Lett. B 690, 57 (2010).

[82] C. B. Braeuninger, A. Ibarra, and C. Simonetto, Phys. Lett. B 692, 189 (2010).

[83] F. J. Botella and J. P. Silva, Phys. Rev. D 51, 3870 (1995).

[84] S. Pakvasa and H. Sugawara, Phys. Lett. 73B, 61 (1978).

[85] D. Wyler, Phys. Rev. D 19, 330 (1979).

[86] M. Nebot and J. P. Silva, Phys. Rev. D 92, 085010 (2015).

[87] W. Grimus and L. Lavoura, Eur. Phys. J. C 39, 219 (2005).

[88] T. P. Cheng, E. Eichten, and L.-F. Li, Phys. Rev. D 9, 2259 (1974).

[89] M. Jung, A. Pich, and P. Tuzon, J. High Energy Phys. 11 (2010) 003.

[90] J. F. Gunion and H. E. Haber, Phys. Rev. D 67, 075019 (2003).

[91] G. Aad et al. (ATLAS and CMS Collaborations), J. High Energy Phys. 08 (2016) 045. 
[92] J. F. Gunion, H. E. Haber, G. L. Kane, and S. Dawson, The Higgs Hunter's Guide, Front. Phys., Vol. 80 (Avalon Publishing, New York, 2000).

[93] M. Spira, A. Djouadi, D. Graudenz, and P. M. Zerwas, Nucl. Phys. B453, 17 (1995).

[94] S. Dittmaier et al. (LHC Higgs Cross Section Working Group Collaboration), arXiv:1101.0593.

[95] S. Dittmaier et al. (LHC Higgs Cross Section Working Group Collaboration), arXiv:1201.3084.

[96] S. Heinemeyer et al. (LHC Higgs Cross Section Working Group Collaboration), arXiv:1307.1347.

[97] D. de Florian et al. (LHC Higgs Cross Section Working Group Collaboration), arXiv:1610.07922.

[98] Z.-z. Xing, H. Zhang, and S. Zhou, Phys. Rev. D 86, 013013 (2012).

[99] H. Georgi, S. Glashow, M. Machacek, and D. V. Nanopoulos, Phys. Rev. Lett. 40, 692 (1978).

[100] Y. Zhou, Phys. Rev. D 93, 013019 (2016).

[101] G. Perez, Y. Soreq, E. Stamou, and K. Tobioka, Phys. Rev. D 93, 013001 (2016).

[102] F. Yu, J. High Energy Phys. 02 (2017) 083.

[103] J. Cohen, S. Bar-Shalom, G. Eilam, and A. Soni, Phys. Rev. D 97, 055014 (2018).

[104] A. D. Martin, W. J. Stirling, R. S. Thorne, and G. Watt, Eur. Phys. J. C 63, 189 (2009).

[105] M. Aaboud et al. (ATLAS Collaboration), J. High Energy Phys. 12 (2017) 024.

[106] A. M. Sirunyan et al. (CMS Collaboration), Phys. Lett. B 780, 501 (2018).
[107] A. M. Sirunyan et al. (CMS Collaboration), Phys. Lett. B 779, 283 (2018).

[108] N. Kauer and G. Passarino, J. High Energy Phys. 08 (2012) 116.

[109] G. Aad et al. (ATLAS Collaboration), Eur. Phys. J. C 75, 335 (2015).

[110] V. Khachatryan et al. (CMS Collaboration), J. High Energy Phys. 09 (2016) 051.

[111] V. Khachatryan et al. (CMS Collaboration), Phys. Lett. B 744, 184 (2015).

[112] M. Aaboud et al. (ATLAS Collaboration), Phys. Rev. Lett. 119, 051802 (2017).

[113] S. M. Barr and A. Zee, Phys. Rev. Lett. 65, 21 (1990); 65, 2920(E) (1990).

[114] R. G. Leigh, S. Paban, and R. M. Xu, Nucl. Phys. B352, 45 (1991).

[115] J. F. Gunion and R. Vega, Phys. Lett. B 251, 157 (1990).

[116] D. Chang, W.-Y. Keung, and T. C. Yuan, Phys. Rev. D 43, R14 (1991).

[117] C. Kao and R.-M. Xu, Phys. Lett. B 296, 435 (1992).

[118] V. Ilisie, J. High Energy Phys. 04 (2015) 077.

[119] S. Inoue, M. J. Ramsey-Musolf, and Y. Zhang, Phys. Rev. D 89, 115023 (2014).

[120] W. Altmannshofer, J. Brod, and M. Schmaltz, J. High Energy Phys. 05 (2015) 125.

[121] L. Bian, T. Liu, and J. Shu, Phys. Rev. Lett. 115, 021801 (2015).

[122] M. Jung and A. Pich, J. High Energy Phys. 04 (2014) 076. 